\title{
ALMA Observations of the Physical and Chemical Conditions in Centaurus A
}

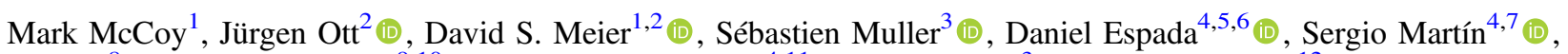
Frank P. Israel ${ }^{8}$ (D) , Christian Henkel $^{9,10}$ (10), Violette Impellizzeri ${ }^{4,11}$, Susanne Aalto ${ }^{3}$, Philip G. Edwards ${ }^{12}$, Andreas Brunthaler ${ }^{9}$ (i), Nadine Neumayer ${ }^{13}$ (i), Alison B. Peck ${ }^{11}$ (D), Paul van der Werf ${ }^{8}$, and Ilana Feain ${ }^{14}$

${ }^{1}$ New Mexico Institute of Mining and Technology, 801 Leroy Place, Socorro, NM, 87801, USA; Mark.McCoy@student.nmt.edu

${ }^{2}$ National Radio Astronomy Observatory, Pete V. Domenici Array Science Center, P.O. Box O, Socorro, NM, 87801, USA

${ }^{3}$ Department of Earth, Space and Environment, Chalmers University of Technology, Onsala Space Observatory, SE-43992 Onsala, Sweden 4 Joint Alma Observatory, Alonso de Córdova, 3107, Vitacura, Santiago 763-0355, Chile

${ }^{5}$ National Astronomical Observatory of Japan, 2-21-1 Osawa, Mitaka, Tokyo 181-8588, Japan

${ }^{6}$ The Graduate University for Advanced Studies (SOKENDAI), 2-21-1 Osawa, Mitaka, Tokyo 181-0015, Japan

${ }^{7}$ European Southern Observatory, Alonso de Córdova 3107, Vitacura, Santiago, Chile

${ }^{8}$ Leiden Observatory, Leiden University, P.O. Box 9513, NL-2300 RA Leiden, The Netherlands

${ }_{9}$ Max-Planck-Institut für Radioastronomie, Auf dem Hügel 69, D-53121 Bonn, Germany

10 Astronomy Department, King Abdulaziz University, P.O. Box 80203, Jeddah 21589, Saudi Arabia

${ }^{11}$ National Radio Astronomy Observatory, 520 Edgemont Road, Charlottesville, VA 22903, USA

${ }^{12}$ CSIRO CASS/ATNF, P.O. Box 76 Epping, NSW 1710, Australia

13 Max-Planck Institut für Astronomie, Königstuhl 17, D-69117 Heidelberg, Germany

${ }^{14}$ School of Physics, University of Sydney, NSW 2006, Australia

Received 2016 August 24; revised 2017 October 18; accepted 2017 October 30; published 2017 December 14

\begin{abstract}
Centaurus A, with its gas-rich elliptical host galaxy, NGC 5128, is the nearest radio galaxy at a distance of 3.8 Mpc. Its proximity allows us to study the interaction among an active galactic nucleus, radio jets, and molecular gas in great detail. We present ALMA observations of low- $J$ transitions of three CO isotopologues, $\mathrm{HCN}, \mathrm{HCO}^{+}, \mathrm{HNC}, \mathrm{CN}$, and $\mathrm{CCH}$ toward the inner projected $500 \mathrm{pc}$ of NGC 5128. Our observations resolve physical sizes down to $40 \mathrm{pc}$. By observing multiple chemical probes, we determine the physical and chemical conditions of the nuclear interstellar medium of NGC 5128. This region contains molecular arms associated with the dust lanes and a circumnuclear disk (CND) interior to the molecular arms. The CND is approximately $400 \mathrm{pc}$ by $200 \mathrm{pc}$ and appears to be chemically distinct from the molecular arms. It is dominated by dense gas tracers while the molecular arms are dominated by ${ }^{12} \mathrm{CO}$ and its rare isotopologues. The $\mathrm{CND}$ has a higher temperature, elevated $\mathrm{CN} / \mathrm{HCN}$ and $\mathrm{HCN} / \mathrm{HNC}$ intensity ratios, and much weaker ${ }^{13} \mathrm{CO}$ and $\mathrm{C}^{18} \mathrm{O}$ emission than the molecular arms. This suggests an influence from the AGN on the CND molecular gas. There is also absorption against the AGN with a low velocity complex near the systemic velocity and a high velocity complex shifted by about $60 \mathrm{~km} \mathrm{~s}^{-1}$. We find similar chemical properties between the CND in emission and both the low and high velocity absorption complexes, implying that both likely originate from the CND. If the HV complex does originate in the CND, then that gas would correspond to gas falling toward the supermassive black hole.
\end{abstract}

Key words: astrochemistry - galaxies: active - galaxies: elliptical and lenticular, cD - galaxies: individual (NGC 5128, Centaurus A) - galaxies: ISM - galaxies: structure

Supporting material: machine-readable table

\section{Introduction}

Radio galaxies are the largest contribution to the extragalactic radio sky above $1 \mathrm{mJy}$ (Condon 1984). Radio galaxies are fairly rare in the local universe. Most radio galaxies are of the elliptical type (e.g., Wilson \& Colbert 1995) and frequently show dust features (e.g., Verdoes Kleijn et al. 1999). In the nuclear regions of some radio galaxies, $\mathrm{CO}$ rotational transitions are observed with double-horned profiles indicative of rotating disks (e.g., Leon et al. 2003; Lim et al. 2003). Mergers with small gas-rich galaxies may be the source of cold gas in evolved elliptical galaxies (e.g., Salomé \& Combes 2003; Davis et al. 2011; O'Sullivan et al. 2015).

The power source of the active galactic nuclei (AGNs) and the giant synchrotron jets/lobes in radio galaxies is thought to be the accretion of material onto the central supermassive black hole. The mechanisms for transporting gas from the outer kiloparsecs to the central parsecs of radio galaxies are, however, not well understood. In turn, the AGN and jets also impact their environment, possibly quenching or inducing star formation activity, and launching material out of the galaxy (e.g., Meijerink et al. 2007; Aalto 2008; Ostriker et al. 2010; Ott et al. 2013).

High spatial resolution observations are required to determine the nature and properties of the gas in the nuclear region of radio galaxies. NGC 5128 is the nearest radio galaxy (3.8 Mpc; Harris et al. 2010). Its proximity makes NGC 5128 an important source for further understanding the details of accretion onto SMBHs.

NGC 5128 is the host galaxy of the strong radio source Centaurus A (hereafter Cen A; see Israel 1998 for a comprehensive review). The $5.5 \times 10^{7} M_{\odot}$ supermassive black hole at the center of NGC 5128 (Cappellari et al. 2009; Neumayer 2010) is powering synchrotron jets, which create the inner and outer radio lobe structures of Cen A. The inner lobes extend roughly 12 arcminutes on the sky and are much brighter than the outer lobes (Feain et al. 2011), which extend 8 degrees on the sky. The synchrotron jet in Cen $\mathrm{A}$ has been observed to interact with the gas and to induce star formation (SF; e.g., Charmandaris et al. 2000; 
Auld et al. 2012; Crockett et al. 2012; Santoro et al. 2015; Salomé et al. 2016).

The history of NGC 5128 is dominated by multiple merger events, which have left signatures in the form of a system of optical shells (e.g., Malin et al. 1983; Peng et al. 2002). The galaxy is also bisected by a prominent dust lane. Presumably, the merger events that produced the optical shells are also responsible for the dust lane through multiple warps and gas re-accretion. Modeling of warps in the gas, from $6.5 \mathrm{kpc}$ down to the inner $2 \mathrm{pc}$, have been compiled by Quillen et al. (2006, 2010). The warped disk model has many kinks that give rise to the various morphological and kinematic structures observed in Cen A - most prominently, a projected parallelogram structure detected in the IR that is associated with the molecular arms (detected in $\mathrm{H} \alpha$ : Nicholson et al. 1992; NIR: Quillen et al. 1993; Kainulainen et al. 2009; Sub mm continuum: Hawarden et al. 1993; Leeuw et al. 2002; CO: Phillips et al. 1987; Eckart et al. 1990; Rydbeck et al. 1993; Liszt 2001; Espada et al. 2009; Mid-IR: Laurent et al. 1999; Quillen et al. 2006). $\mathrm{H} \alpha$ emission detected in this putative warped disk indicates that massive stars are currently forming (e.g., Nicholson et al. 1992). A recent study, however, suggests that the gas associated with the dust parallelogram may also be described by spiral arms in addition to a warped disk (Espada et al. 2012). Interior to the parallelogram structure is a 405 pc by 215 pc circumnuclear disk with a different position angle and inclination (Israel et al. 1990, 1991; Israel 1992; Rydbeck et al. 1993; Neumayer et al. 2007; Quillen et al. 2010).

Espada et al. (2017) describe additional molecular components on much smaller scales within the circumnuclear disk, including CND filaments, a nuclear ring, nuclear filaments (probably shocks), a nuclear disk ( $\sim 30 \mathrm{pc}$ in size), and absorption against the AGN. The nuclear disk is detected in ionized and molecular hydrogen (Marconi et al. 2001, 2006; Neumayer et al. 2007). The absorption profile consists of a low velocity complex near the systemic velocity and a high velocity complex shifted by $\sim 60 \mathrm{~km} \mathrm{~s}^{-1}$ (Wiklind \& Combes 1997; Muller et al. 2009). The location of the gas that gives rise to these absorption features is still debated. The absorption has been detected in HI (e.g., Roberts 1970; van der Hulst et al. 1983; Sarma et al. 2002; Espada et al. 2011) and molecular lines (e.g., Israel et al. 1990; Wiklind \& Combes 1997; Eckart et al. 1999; Espada et al. 2011).

This paper focuses on the kinematics, dynamics, and chemistry of the disk-like structure associated with the dust lane, referred to as the molecular arm feature, and the circumnuclear disk (CND). The sensitivity and resolution of the Atacama Large Millimeter/Submillimeter Array (ALMA) allow us to test the physical and chemical conditions in such an extreme environment. We present a multi-line survey to derive the nuclear gas conditions and to study the impact of the AGN on the nuclear gas. Section 2 describes the ALMA observations and the data reduction. Section 3 presents results from the observations. Section 4 focuses on describing the dynamics, masses, and chemistry of the molecular gas, a comparison of the transitions seen in both emission and absorption, and possible signatures AGN influence on these spectra. Section 5 summarizes our results.

\section{Observations and Data Reduction}

\subsection{Observational Setup}

Observations were taken with ALMA during Cycle 0 early science observing under the project code 2011.0.00010.S (PI: Ott). We observed 20 spectral windows, four in the $1 \mathrm{~mm}$ band (ALMA Band 6), and 16 in the $3 \mathrm{~mm}$ band (ALMA Band 3). The observation and image parameters are outlined in Table 1.

The $1 \mathrm{~mm}$ observations were carried out in a single run with 17 antennas in a compact configuration on 2012 January 24, with baselines of $18 \mathrm{~m}$ to $125 \mathrm{~m}$. The maximum recoverable scale is $10^{\prime \prime}$ and the primary beam is $27^{\prime \prime}$. Titan is the flux and bandpass calibrator, and complex gain (phase/gain) calibration was done by observing PKS B1424-418 (J1427-4206) every 10 minutes. There were no significant spectral lines in the atmosphere of Titan that may contaminate the Cen A spectra. The total on-source integration time is $\sim 33$ minutes, with each spectral window separated into 3840 channels with spacings of $244.14 \mathrm{kHz}$ per channel corresponding to a velocity resolution of $0.66 \mathrm{~km} \mathrm{~s}^{-1}$ after Hanning smoothing and a bandwidth of 937.5 MHz (1260 $\left.\mathrm{km} \mathrm{s}^{-1}\right)$.

The $3 \mathrm{~mm}$ band observations were taken in multiple runs spanning the time between 2012 April 7 and 2012 May 9 in a more extended configuration with baselines of $36 \mathrm{~m}$ to $400 \mathrm{~m}$. The maximum recoverable scale was $20^{\prime \prime}$, and the primary beam was $62^{\prime \prime}$. The $3 \mathrm{~mm}$ observations were performed with 17 antennas for the first two observing runs and 16 antennas for the final two observations (See Table 1 for details). As with the Band 6 observations, the flux and complex gain calibrators are Titan and PKS B1424-418 (J1427-4206), respectively. The phase calibrator was observed every 16 minutes. 3C279 (J1256 -0547) was used as the bandpass calibrator. The total onsource integration time is $\sim 66$ minutes with each spectral window, again divided into 3840 channels but with spacings of $61.04 \mathrm{kHz}$ per channel corresponding to a velocity resolution between 0.32 and $0.44 \mathrm{~km} \mathrm{~s}^{-1}$ with a bandwidth of $234.4 \mathrm{MHz}$ (approximately $730 \mathrm{~km} \mathrm{~s}^{-1}$ ).

The configurations were selected such that the maximum recoverable scales are approximately the same between the $3 \mathrm{~mm}$ and $1 \mathrm{~mm}$ bands, so comparisons between the fluxes in each band should, in principle, be robust on the sampled scales.

\subsection{Calibration and Imaging}

Initial calibration and editing of the data were performed by the ALMA team using the CASA (Common Astronomy Software Applications) data reduction package (McMullin et al. 2007). After delivery, self-calibration and imaging were performed by us with CASA version 4.1. We complemented our data with the Science Verification ${ }^{12} \mathrm{CO}(2-1)$ observations of Cen A, giving us a total of nine molecular transitions with emission detected in the nuclear region.

We performed a self-calibration on the bright radio continuum core of Cen A using the CASA task GAINCAL to generate the calibration tables and APPLYCAL to apply the corrections to the data. The calibration tables were made by carefully avoiding channels with line emission. As the first step, we self-calibrated the phases only; then we did an iteration of simultaneous amplitude and phase self-calibration. We then used the CASA task UVCONTSUB2 to fit a first order polynomial continuum model to channels that were free of both line emission and absorption. This model was then subtracted 
Table 1

Observational Parameters

\begin{tabular}{|c|c|c|c|c|c|c|c|c|}
\hline $\begin{array}{l}\text { Center Freq. } \\
(\mathrm{GHz}) \\
(1)\end{array}$ & $\begin{array}{c}\text { Bandwidth } \\
\text { (MHz) }\left[\mathrm{km} \mathrm{s}^{-1}\right] \\
\text { (2) }\end{array}$ & $\begin{array}{c}\Delta v \\
\left(\mathrm{~km} \mathrm{~s}^{-1}\right) \\
(3)\end{array}$ & $\begin{array}{l}\text { Date } \\
\text { (4) }\end{array}$ & $\begin{array}{c}\text { Beam } \\
\left(“ \times,{ }^{\circ}\right) \\
(5)\end{array}$ & $\begin{array}{l}\text { Channel rms } \\
\left(\mathrm{mJy}^{\text {beam }}{ }^{-1}\right) \\
(6)\end{array}$ & $\begin{array}{l}\text { Cont. Flux Density } \\
\text { (Jy) } \\
\text { (7) }\end{array}$ & $\begin{array}{l}\text { Spectral } \\
\text { Line ID } \\
(8)\end{array}$ & $\begin{array}{l}\text { Rest Freq. } \\
\quad(\mathrm{GHz}) \\
(9)\end{array}$ \\
\hline \multicolumn{9}{|c|}{ Band $3(3 \mathrm{~mm})$} \\
\hline 85.338706 & $234.4[806]$ & 0.420 & 2012 Apr 07 & $2.07 \times 1.69,-76.2$ & 4.1 & 7.5 & & \\
\hline 86.846824 & $234.4[822]$ & 0.428 & 2012 Apr 07 & $2.19 \times 1.67,-83.7$ & 4.8 & 7.4 & & \\
\hline 87.169498 & $234.4[806]$ & 0.420 & 2012 May 08 & $2.19 \times 1.61,72.2$ & 4.8 & 7.8 & $\mathbf{C C H}\left(\mathbf{N}=\mathbf{1 - 0} \mathbf{0} \mathbf{J}=\frac{3}{2}-\frac{1}{2}\right)$ & 87.316925 \\
\hline 87.765057 & 234.4 [799] & 0.416 & 2012 May 08 & $2.17 \times 1.60,73.8$ & 4.2 & 7.9 & $\mathrm{HNCO}\left(4_{04}-3_{03}\right)$ & 87.925238 \\
\hline 88.470137 & 234.4 [791] & 0.412 & 2012 May 08 & $2.13 \times 1.59,74.6$ & 4.0 & 7.4 & $\operatorname{HCN}(1-0)$ & 88.631847 \\
\hline 89.026047 & $234.4[787]$ & 0.410 & 2012 May 08 & $2.12 \times 1.57,74.8$ & 4.2 & 7.9 & $\mathrm{HCO}^{+}(\mathbf{1}-\mathbf{0})$ & 89.188526 \\
\hline 90.498407 & $234.4[776]$ & 0.404 & 2012 May 08 & $1.66 \times 1.61,-59.0$ & 4.4 & 8.1 & $\operatorname{HNC}(1-0)$ & 90.663564 \\
\hline 90.813277 & 234.4 [772] & 0.402 & 2012 May 08 & $1.66 \times 1.61,-46.1$ & 3.9 & 8.1 & $\mathrm{HC}_{3} \mathrm{~N}(10-9)$ & 90.978989 \\
\hline 91.803617 & $234.4[764]$ & 0.398 & 2012 May 08 & $1.66 \times 1.58,-56.6$ & 4.0 & 8.2 & $\mathrm{CH}_{3} \mathrm{CN}\left(5_{3}-4_{3}\right)$ & 91.971160 \\
\hline 93.003656 & $234.4[753]$ & 0.392 & 2012 May 08 & $1.63 \times 1.55,-73.0$ & 3.8 & 8.2 & $\mathrm{~N}_{2} \mathrm{H}^{+}(1-0)$ & 93.173777 \\
\hline 97.979577 & $234.4[714]$ & 0.372 & 2012 Apr 07 & $1.68 \times 1.45,-59.4$ & 4.6 & 7.6 & & \\
\hline 100.076592 & $234.4[703]$ & 0.366 & 2012 Apr 07 & $1.88 \times 1.50,-70.9$ & 5.1 & 7.6 & & \\
\hline 109.590043 & $234.4[641]$ & 0.334 & 2012 May 07 & $1.38 \times 1.35,-73.7$ & 5.3 & 9.0 & $\mathrm{C}^{18} \mathrm{O}(1-0)$ & 109.782176 \\
\hline 110.001195 & $234.4[637]$ & 0.332 & 2012 May 07 & $1.41 \times 1.32,-72.9$ & 4.6 & 9.0 & ${ }^{13} \mathrm{CO}(1-0)$ & 110.201354 \\
\hline 112.154164 & $234.4[626]$ & 0.326 & 2012 May 07 & $1.38 \times 1.30,-54.1$ & 5.4 & 9.1 & $\mathrm{C}^{17} \mathrm{O}(1-0)$ & 112.358988 \\
\hline 113.230037 & $234.4[618]$ & 0.322 & 2012 May 07 & $1.38 \times 1.27,-69.7$ & 6.1 & 9.2 & $\mathbf{C N}\left(\mathbf{N}=\mathbf{1}-\mathbf{0} ; \mathbf{J}=\frac{3}{2}-\frac{1}{2}\right)$ & 113.490982 \\
\hline \multicolumn{9}{|c|}{ Band $6(1 \mathrm{~mm})$} \\
\hline 218.077608 & $937.5[1286]$ & 0.670 & 2012 Jan 24 & $2.22 \times 1.43,-44.4$ & 4.0 & 6.5 & $\mathrm{H}_{2} \mathrm{CO}\left(3_{03}-2_{02}\right)$ & 218.222195 \\
\hline 219.152815 & $937.5[1279]$ & 0.666 & 2012 Jan 24 & $2.22 \times 1.43,-44.4$ & 4.3 & 6.5 & $\mathrm{C}^{18} \mathrm{O}(2-1)$ & 219.560358 \\
\hline 219.397838 & $937.5[1279]$ & 0.666 & 2012 Jan 24 & $2.23 \times 1.45,-46.0$ & 4.7 & 6.5 & $\operatorname{HNCO}\left(10_{010}-9_{09}\right)$ & 219.798282 \\
\hline 220.007315 & $937.5[1275]$ & 0.664 & 2012 Jan 24 & $2.23 \times 1.46,-44.6$ & 3.9 & 6.8 & ${ }^{13} \mathrm{CO}(2-1)$ & 220.398684 \\
\hline
\end{tabular}

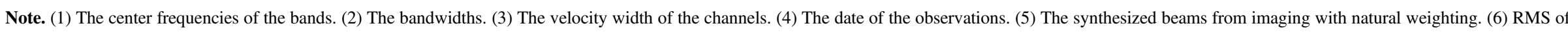

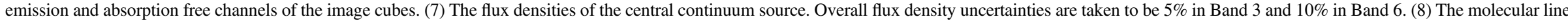

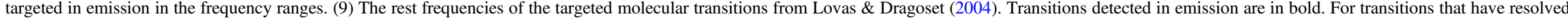
hyperfine structure, the strongest of the hyperfine is reported. 
from the $(u, v)$ data to create a continuum-free spectral line data set for each line.

With the continuum emission removed, we then proceeded to image each of the spectral windows. Imaging was performed using the CASA task CLEAN with natural weighting and an image size of $320 \times 320$ pixels at 0.2 arcseconds per pixel. This corresponds to an image size of $64^{\prime \prime}$, which encompasses the primary beams of both the Band 3 (62" primary beam) and the Band 6 (27" primary beam) observations. The synthesized beams for the images are listed in Table 1. Continuum images were made using the line-free channels of the two representative frequency ranges, centered on $220.007 \mathrm{GHz}$ and $90.498 \mathrm{GHz}$, with synthesized beams listed in Table 1. An overall flux accuracy of 5\% was assumed for the Band 3 observations, and a flux accuracy of $10 \%$ was assumed for the Band 6 observations. ${ }^{15}$

Each frequency range was imaged at the full spectral resolution ( 3840 channels) to obtain the highest spectral resolution absorption spectra toward the continuum source of Cen A achievable with this data. The image cubes used to make the absorption spectra were not cleaned because of an artifact created during the execution of the CLEAN algorithm. Near the location of the absorption, CLEAN created a positive spike in the image cube as the map transitioned from noise to a deep absorption feature. The clean artifacts are not fully understood, but they appear to be related to the high dynamic range change between adjacent absorption/non-absorption channels. The artifacts are not present in the dirty cube that we used for the analysis of the absorption spectra.

Broader spectral resolution (averaged over 10 channels) image cubes were created and cleaned to map the emission. The emission cubes were then inspected for artifacts created by CLEAN. The artifacts in the emission were all located in the center of the field of view, co-spatial with the absorption, which were already masked due to the absorption hole (discussed later) and so do not affect the displayed emission maps. Therefore, in summary, the artifact does not impact any map/ result presented in this paper. The emission cubes were all then convolved to a common beam of 2 !" $25 \times 2$ !! 25 , which is slightly larger than the original synthesized beam sizes given in Table 1 . The lone exception is the ${ }^{12} \mathrm{CO}(2-1)$ image cube, which due to $\mathrm{u}-\mathrm{v}$ coverage was unable to be convolved to the same beam. In order to compare the ${ }^{12} \mathrm{CO}(2-1)$ and the other isotopologues, the ${ }^{12} \mathrm{CO}(2-1)$ was convolved to a 3 !" $0 \times 3$ !" 0 beam and compared to separate ${ }^{13} \mathrm{CO}(2-1),{ }^{13} \mathrm{CO}(1-0)$, and $\mathrm{C}^{18} \mathrm{O}(2-1)$ images, also convolved to 3 !! $0 \times 3$ !' 0 . Integrated intensity, intensity-weighted velocity, and intensity-weighted velocity dispersion maps were made for each transition with observed emission. A mask was created for each molecular transition by convolving the image cube with a 4 ". $5 \times 4$." 5 beam (twice the size of the synthesized beam of the image cube) and then only including emission in the original image cube where the smoothed cube contained emission stronger than $\sim 3$ times the rms. This clipping was necessary to minimize the effect of the absorption near the radio core on the rest of the CND emission. This mask was applied to the image cube during the map making process. In addition, a mask of $\sim 5^{\prime \prime} \times 5^{\prime \prime}$ was used to mask the absorption at the center for each of the moment maps.

\footnotetext{
15 From ALMA cycle 0 capabilities. https://almascience.eso.org/documents-andtools/cycle-0/capabilities/at_download/file\&usg=AOvVaw3gee33Ts0yieHWve 2ubbZs.
}

Additional ALMA data obtained during science verification (2011.0.00008.SV) were used to provide the ${ }^{12} \mathrm{CO}(2-1)$ $1.3 \mathrm{~mm}$ transition for comparison. The data were edited, calibrated, and imaged in a consistent manner as the rest of our data for direct comparison. However, the spectral resolution is coarser than our data, at $10 \mathrm{~km} \mathrm{~s}^{-1}$ per channel, while our data were imaged with velocity resolutions ranging from 1.6 to $3.3 \mathrm{~km} \mathrm{~s}^{-1}$ per channel.

\section{Results}

\subsection{Millimeter Continuum}

The AGN is detected as an unresolved millimeter continuum source at a position of R.A.(J2000): $13^{\mathrm{h}} 25^{\mathrm{m}} 27^{\mathrm{s}} .616$ Decl. (J2000): $-43^{\circ} 01^{\prime} 08$ ". 813 . The continuum fluxes for the bright central source were determined from apertures in cleaned continuum images and are listed in column (7) of Table 1. Figure 1 shows the $3 \mathrm{~mm}$ band (at $90.5 \mathrm{GHz}$ ) and $1 \mathrm{~mm}$ band (at $220.0 \mathrm{GHz}$ ) continuum images. The dashed line in Figure 1 (lower panel) shows the half power of the $1.3 \mathrm{~mm}$ primary beam, while the corresponding half power for $3 \mathrm{~mm}$ is outside the displayed field of view. The radio jet is also detected in both bands, but it is stronger in the $3 \mathrm{~mm}$ continuum image. Two knots of the northern jet are detected, but no portion of the southern jet is seen. This is consistent with previous observations. The knots correspond to A1 (inner) and A2 (outer) of Clarke et al. (1992), which were studied in more detail at radio wavelengths and X-ray energies by Hardcastle et al. (2003). Self-calibration was required to achieve the dynamic range necessary to detect the jet in the presence of the strong nuclear source. We achieved rms values for the $3 \mathrm{~mm}$ and $1 \mathrm{~mm}$ images of $1.3 \mathrm{mJy} \mathrm{beam}^{-1}$ and $2.1 \mathrm{mJy}$ beam $^{-1}$, respectively. The jet integrated flux is around $30 \pm$ $5 \mathrm{mJy}$ in band 3 and $20 \pm 5 \mathrm{mJy}$ in band 6 , compared to the core flux of $8.1 \pm 0.4 \mathrm{Jy}$ in band 3 and $6.7 \pm 0.7 \mathrm{Jy}$ in band 6 . Both knots of the jet are well detected in both images, with the outer knot slightly stronger than the inner knot in both bands. The spectral index between the two bands is $-0.2 \pm 0.1$ for the bright radio core continuum, and $-0.4 \pm 0.2$ for the jet continuum, with the spectral index $\alpha$ defined as $S \propto \nu^{\alpha}$. Israel et al. (2008) determine the core continuum spectral index to vary over the range -0.2 to -0.6 , consistent with our value. However, the $3 \mathrm{~mm}$ and $1 \mathrm{~mm}$ continuum were not observed simultaneously. This adds uncertainty to all flux comparisons due to the known $\sim 30 \%$ variability of Centaurus A (See Israel et al. 2008, their Figure 5). This time variability in the flux could potentially explain the flux differences listed in Table 1. However, the fluxes determined for different frequencies in the same observing band give a positive spectral index, so there are likely systemic errors as well.

\subsection{Molecular Gas Structure}

We detected eight molecular transitions in emission toward the nuclear region of Cen $\mathrm{A}$. The detected transitions are the $J=2-1$ rotational transitions of ${ }^{13} \mathrm{CO}$ and $\mathrm{C}^{18} \mathrm{O}$ [Band 6]; the $J=1-0$ rotational transitions of ${ }^{13} \mathrm{CO}, \mathrm{HCO}^{+}, \mathrm{HCN}$, $\mathrm{HNC}$; and the $N=1-0 ; J=\frac{3}{2}-\frac{1}{2}$ transitions of $\mathrm{CN}$ and $\mathrm{CCH}$ (hereafter $\mathrm{CN}(1-0)$ and $\mathrm{CCH}(1-0))$ [Band 3]. In Band $6, \operatorname{HNCO}\left(10_{0,10}-9_{0,9}\right)$ and $\mathrm{H}_{2} \mathrm{CO}\left(3_{0,3}-2_{0,2}\right)$ were targeted but not detected in emission. $\mathrm{HC}_{3} \mathrm{~N}(10-9), \mathrm{CH}_{3} \mathrm{CN}\left(5_{3}-4_{3}\right)$, $\mathrm{HNCO}\left(4_{0,4}-3_{0,3}\right)$, and $J=1-0$ of $\mathrm{C}^{18} \mathrm{O}, \mathrm{C}^{17} \mathrm{O}$, and $\mathrm{N}_{2} \mathrm{H}^{+}$ were targeted in band $3(3 \mathrm{~mm})$, but not detected in emission. 

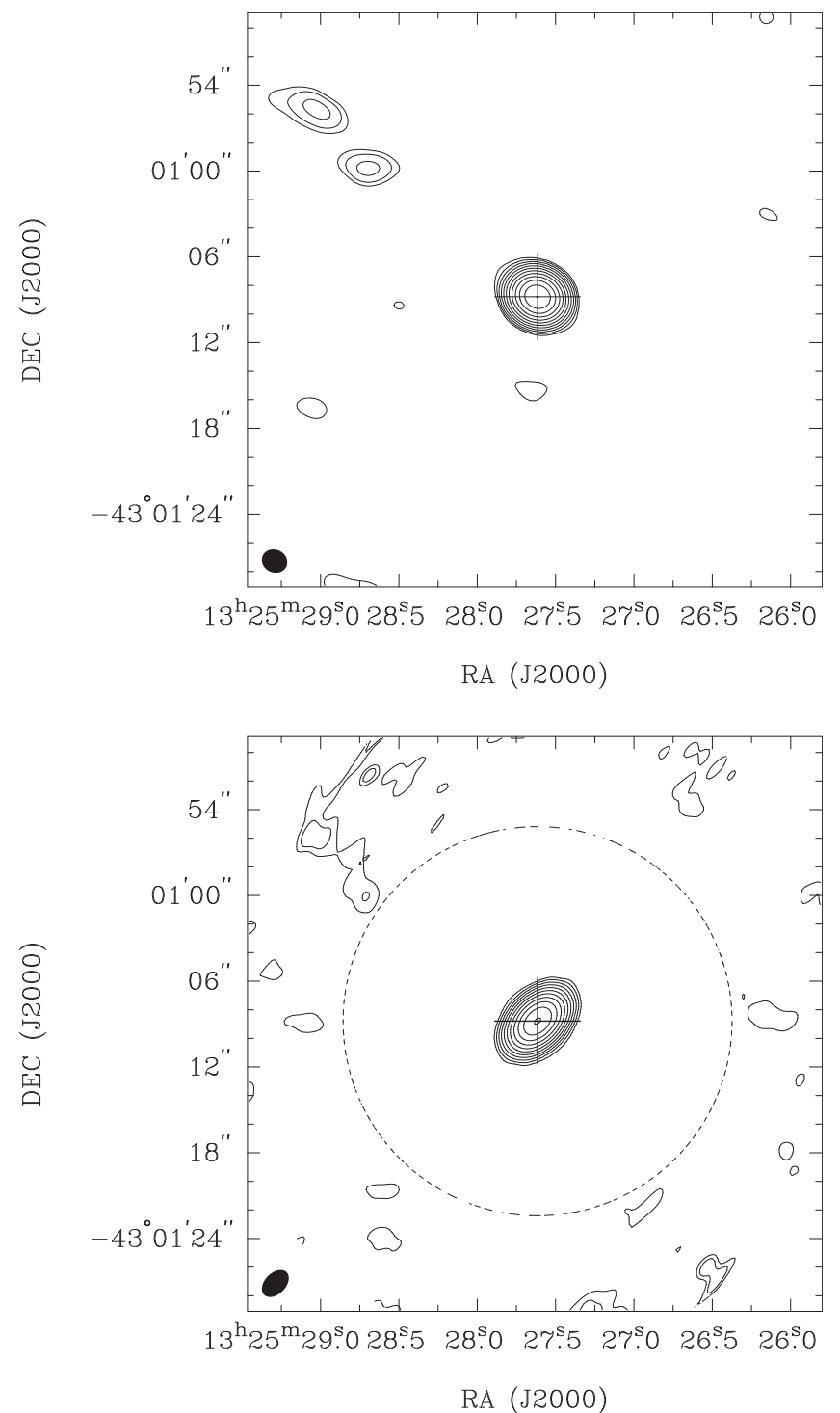

Figure 1. Continuum image for $3 \mathrm{~mm}$ (top) and $1.3 \mathrm{~mm}$ (bottom). The contours for both images are in steps of $2^{n} \cdot 3 \sigma$ with $n=0,1,2, \ldots, 11$ and $\sigma=1.3 \mathrm{mJy}$ beam $^{-1}$ and $2.1 \mathrm{mJy} \mathrm{beam}^{-1}$ for $3 \mathrm{~mm}$ and $1 \mathrm{~mm}$, respectively. Both images show the continuum from the central core as well as 2 knots of the northern jet. The dashed circle in the $1 \mathrm{~mm}$ image shows the $50 \%$ level of the primary beam. The synthesized beams are shown as filled ellipses in the bottom left corner of each plot, 1". $66 \times 1$ !" $61,+59.0$ for $3 \mathrm{~mm}$ and 2 ". $23 \times 1$ ". 46 , $-44^{\circ} .6$ for $1.3 \mathrm{~mm}$. The cross is 6 arcseconds in size $(\sim 110 \mathrm{pc})$ and is centered on the core continuum emission (located at R.A.: $13^{\mathrm{h}} 25^{\mathrm{m}} 27^{\mathrm{s}} .616$ Decl.: $-43^{\circ}$ $01^{\prime} 08$ ". 813 in J2000).

Figure 2 shows the primary beam corrected integrated intensity (moment 0) maps of the detected $\mathrm{CO}$ isotopologues and $\mathrm{HCO}^{+}$transitions toward the nuclear region of Cen $\mathrm{A}$, as well as a comparable ${ }^{12} \mathrm{CO}(2-1)$ moment 0 map from ALMA science verification data. Figure 3 shows the other detected dense gas tracers, $\mathrm{HCN}(1-0), \mathrm{CN}(1-0), \operatorname{HNC}(1-0)$, and $\mathrm{CCH}(1-0)$ toward the same region of Cen $\mathrm{A}$ as in Figure 2. There are two main structures in the maps visible on our observed scales: the two linear molecular arms, and the dense circumnuclear disk (CND).

In each of the detected $\mathrm{CO}$ isotopologue maps, there are two clumpy linear features, referred to as molecular arms, extending roughly east-west, that coincide with the parallelogram-shaped dust lanes. The two linear features are separated by about $15^{\prime \prime}$
$(275 \mathrm{pc})$, each at a projected offset from the SMBH of about 7 ".5. Both molecular arms are roughly parallel and at a position angle of $\sim 113^{\circ}$. The southern arm is brighter than the northern arm in all detected molecular lines, with the brightest clumps of the southern arm $\sim 50 \%$ brighter than the brightest clumps of the northern arm. The molecular arms extend beyond the primary beams of both the $3 \mathrm{~mm}$ band and $1 \mathrm{~mm}$ band observations while maintaining the $15^{\prime \prime}$ separation, which is consistent with observations by Espada et al. (2009).

Figure 4 shows the ${ }^{13} \mathrm{CO}(2-1)$ integrated intensity map in grayscale with $\mathrm{HCO}^{+}(1-0)$ integrated intensity contours overlaid. Eight regions are noted in the figure and are used for analysis of the molecular arms and CND. Region A is part of the northern arm, while regions $\mathrm{F}, \mathrm{G}$, and $\mathrm{H}$ are located along the southern molecular arm. Regions C, D, and E are in the CND. Region B represents a location of overlap between the molecular arms and the CND. The CND is measured to be $\sim 410 \mathrm{pc}\left(22^{\prime \prime}\right) \times 215 \mathrm{pc}(10$ " 7$)$, and the centroid of the CND appears slightly off-center relative to the AGN $(\sim 50 \mathrm{pc})$. Garcia-Burillo et al. (2014) have observed the molecular CND in another nearby AGN, NGC 1068, to be off-centered from its AGN as well.

$\mathrm{HCN}(1-0), \mathrm{HCO}^{+}(1-0), \mathrm{HNC}(1-0), \mathrm{CN}(1-0)$, and $\mathrm{CCH}(1-0)$ emission are all weak or absent in the molecular arms, but present in the $\mathrm{CND}$. $\mathrm{HCO}^{+}$traces the majority of the CND, while the others are only detected in specific regions of the CND. If considered as a thin, flat disk, its axial ratio of $\sim 2$ corresponds to an inclination angle of $\sim 60$ degrees and a position angle of about $150^{\circ}$, nearly perpendicular to the radio jet axis. Thus the minor axis of the CND is at a position angle of $60^{\circ}$. The FWHM (full width at half maximum) line width of the CND is around $40 \mathrm{~km} \mathrm{~s}^{-1}$ for an inner diameter of $140 \mathrm{pc}$ corresponding to $\mathrm{C}$ and $\mathrm{D}$ (Figure 4) and $70 \mathrm{~km} \mathrm{~s}^{-1}$ between diameters of 275 and $325 \mathrm{pc}$ corresponding to $\mathrm{B}$ and $\mathrm{E}$ (Figure 4$)$ measured by $\mathrm{HCO}^{+}(1-0)$. The outer portions of the CND for these transitions are coincident with the edges of the CND that are seen in ${ }^{13} \mathrm{CO}(2-1)$. Regions $\mathrm{C}$ and $\mathrm{D}$ are most likely composed of a combination of nuclear filaments and the nuclear ring structure (Espada et al. 2017).

In the ${ }^{12} \mathrm{CO}(2-1)$ and ${ }^{13} \mathrm{CO}(2-1)$ images, the outer edge of the CND is also detected; however, no part of the CND is detected in either ${ }^{13} \mathrm{CO}(1-0)$ or $\mathrm{C}^{18} \mathrm{O}(2-1)$. The clumps in the molecular arms have spectral line widths (FWHM) of $\sim 15 \mathrm{~km} \mathrm{~s}^{-1}$, while the detected portions of the CND have FWHM of $\sim 40 \mathrm{~km} \mathrm{~s}^{-1}$ as measured by ${ }^{13} \mathrm{CO}(2-1)$ (determined in Section 3.4.1), which are consistent with the measured line widths from the dense gas tracers.

The CND is considerably brighter along its major axis than its minor axis. The minor axis at a position angle of approximately $60^{\circ}$ separates regions $\mathrm{B}$ and $\mathrm{C}$ on the northern side from $\mathrm{D}$ and $\mathrm{E}$ on the southern side. From the $\mathrm{HCO}^{+}(1-0)$ emission, the southern side of the minor axis is almost not present, and the northern side of the minor axis is weaker than any of the emission along the CND's major axis. The underluminous minor axis may be due to deviations from a uniform disk, and the underluminous minor axis was also observed in ${ }^{12} \mathrm{CO}(2-1)$, with the SMA as reported by Espada et al. (2009).

\subsection{Molecular Gas Kinematics}

The intensity-weighted mean velocity (moment 1) and intensity-weighted velocity dispersion (moment 2) maps are 

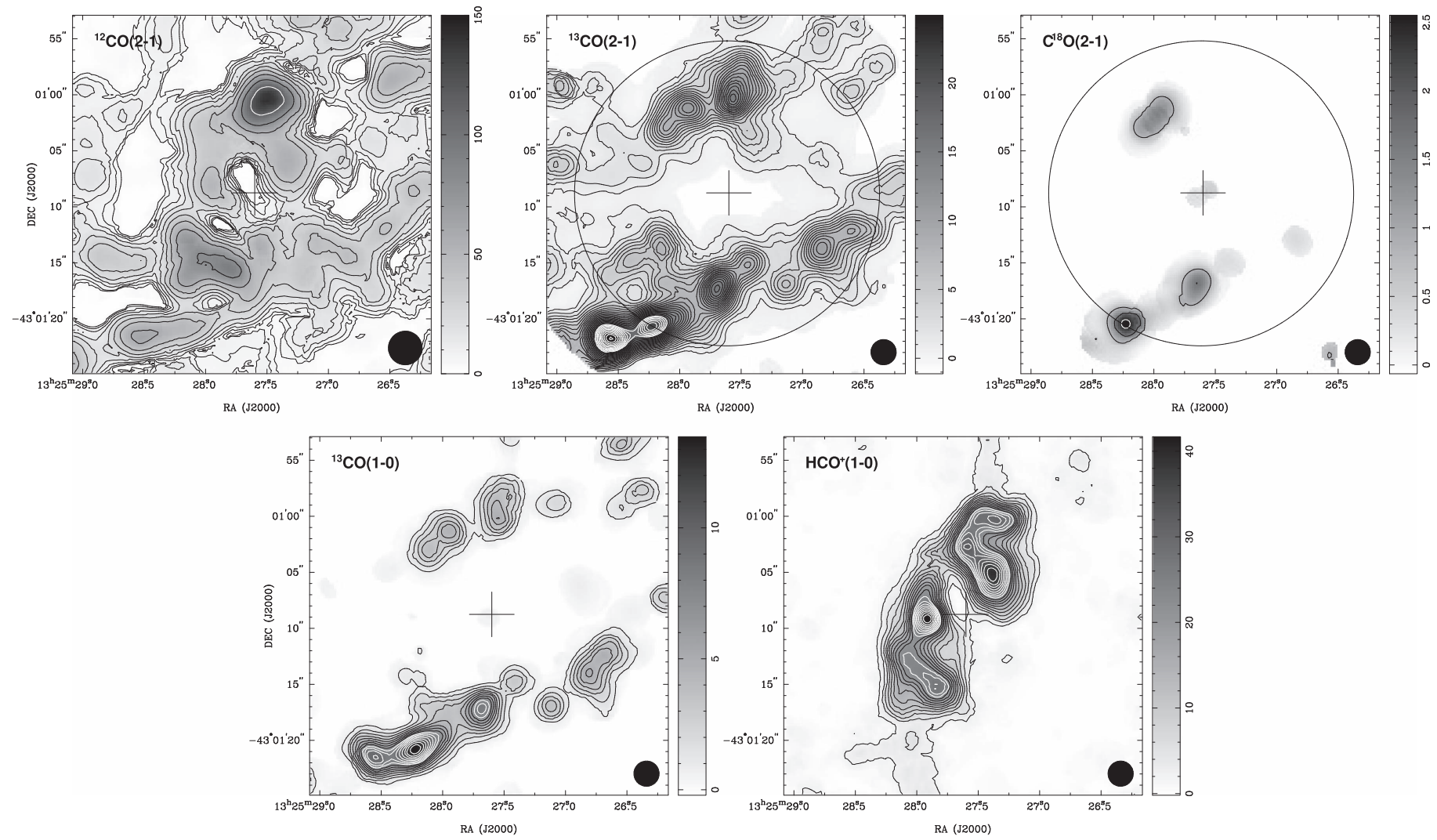

Figure 2. Integrated intensity maps of $\mathrm{CO}$ and its isotopologues are shown in this figure, along with $\mathrm{HCO}^{+}(1-0)$. The top left panel presents ${ }^{12} \mathrm{CO}(2-1)$, the top middle panel presents ${ }^{13} \mathrm{CO}(2-1)$, the top right panel presents $\mathrm{C}^{18} \mathrm{O}(2-1)$, the bottom left panel presents ${ }^{13} \mathrm{CO}(1-0)$, and the bottom right panel presents $\mathrm{HCO}^{+}(1-0)$. The central cross in each of the images identifies the location of the central black hole (located at R.A.: $13^{\mathrm{h}} 25^{\mathrm{m}} 27^{\mathrm{s}} .616$ Decl.: $-43^{\circ} 01^{\prime} 08^{\prime \prime} .813$ in J2000). The size of the cross is $4^{\prime \prime}$ corresponding to about $70 \mathrm{pc}$ at the adopted distance of $3.8 \mathrm{Mpc}$ to Centaurus A. All maps except ${ }^{12} \mathrm{CO}(2-1)$ are at an angular resolution of 2 !' $25 \times 2$ !' 25 , while ${ }^{12} \mathrm{CO}(2-1)$ is at a resolution of $3^{\prime \prime} \times 3^{\prime \prime}$. The contours of the ${ }^{12} \mathrm{CO}(2-1)$ map are determined as $5^{*} 2^{n / 2} \mathrm{~K} \mathrm{~km} \mathrm{~s}{ }^{-1}$ (chosen this way to show the high signal to noise of the map). The white contour on this map is at roughly $113 \mathrm{~K} \mathrm{~km} \mathrm{~s}^{-1}$. The rare CO isotopologues all share the common spacing between contours of $0.80 \mathrm{~K} \mathrm{~km} \mathrm{~s}^{-1}$ corresponding to about $2 \sigma$ in ${ }^{13} \mathrm{CO}(1-0)$ and $4 \sigma$ in ${ }^{13} \mathrm{CO}(2-1)$ and $\mathrm{C}^{18} \mathrm{O}(2-1)$. The first white contours in the ${ }^{13} \mathrm{CO}(2-1)$, ${ }^{13} \mathrm{CO}(1-0)$, and $\mathrm{C}^{18} \mathrm{O}(2-1)$ maps are $16.8,7.2$, and $2.4 \mathrm{~K} \mathrm{~km} \mathrm{~s}^{-1}$, respectively. The $\mathrm{HCO}^{+}(1-0)$ integrated intensity map has a contour spacing of $2.1 \mathrm{~K} \mathrm{~km} \mathrm{~s}^{-1}$, and the first white contour is at a value of $23.1 \mathrm{~K} \mathrm{~km} \mathrm{~s}^{-1}$.

shown in Figure 5, for the $\mathrm{HCO}^{+}(1-0),{ }^{13} \mathrm{CO}(1-0)$, and ${ }^{13} \mathrm{CO}(2-1)$ transitions. The $\mathrm{HCO}^{+}(1-0)$ emission traces the CND very well in both velocity and intensity, but there is no emission from the arms. The ${ }^{13} \mathrm{CO}(1-0)$ emission, on the other hand, traces the arms but not the CND. ${ }^{13} \mathrm{CO}(2-1)$ exhibits both the molecular arms and the CND components and resembles a combination of the $\mathrm{HCO}^{+}(1-0)$ and ${ }^{13} \mathrm{CO}(1-0)$ velocity maps. The ${ }^{13} \mathrm{CO}(1-0)$ transition covers velocities of $\sim 500-600 \mathrm{~km} \mathrm{~s}^{-1}$, and shows a velocity gradient of $\sim 0.2 \mathrm{~km}$ $\mathrm{s}^{-1} \mathrm{pc}^{-1}$ increasing from east to west, with a velocity dispersion of $<10 \mathrm{~km} \mathrm{~s}^{-1}$. $\mathrm{HCO}^{+}(1-0)$ covers the velocity range from about 330 to $770 \mathrm{~km} \mathrm{~s}^{-1}$, and reveals a much steeper velocity gradient along the same axis of $1.2 \mathrm{~km} \mathrm{~s}^{-1}$ $\mathrm{pc}^{-1}$, with a dispersion of $\sim 50 \mathrm{~km} \mathrm{~s}^{-1}$. Using the central velocity of the CND as observed by $\operatorname{HCO}^{+}(1-0)$, we calculate that the systemic velocity in the LSRK (local standard of rest, kinematic definition) velocity frame for Cen A is approximately $550 \mathrm{~km} \mathrm{~s}^{-1}$, which is consistent with the values in the literature, ranging from about 530 to $560 \mathrm{~km} \mathrm{~s}^{-1}$ (Quillen et al. 1992; Marconi et al. 2001; Häring-Neumayer et al. 2006).

Figure 5 also shows the full field ${ }^{13} \mathrm{CO}(1-0)$ intensityweighted mean velocity and velocity dispersion maps. These maps show that outside the field limited by the ${ }^{13} \mathrm{CO}(2-1)$ primary beam, the ${ }^{13} \mathrm{CO}(1-0)$ continues to follow a similar trend of lower dispersion and a smaller velocity gradient than $\mathrm{HCO}^{+}(1-0)$.

The molecular arm component in the ${ }^{13} \mathrm{CO}(2-1)$ moment 1 map ranges from $450 \mathrm{~km} \mathrm{~s}^{-1}$ up to $600 \mathrm{~km} \mathrm{~s}^{-1}$ (similar to the range of ${ }^{13} \mathrm{CO}(1-0)$ ), while the circumnuclear component of the ${ }^{13} \mathrm{CO}(2-1)$ map covers $375 \mathrm{~km} \mathrm{~s}^{-1}$ to $675 \mathrm{~km} \mathrm{~s}^{-1}$ (similar to the range of $\left.\mathrm{HCO}^{+}(1-0)\right)$. There are two locations in the map, near B and E, where the dispersion jumps to $>60 \mathrm{~km} \mathrm{~s}^{-1}$ but the dispersion remains around $\sim 10 \mathrm{~km} \mathrm{~s}^{-1}$ across the remaining map. This jump in the dispersion is not due to a single component with a dispersion of $60 \mathrm{~km} \mathrm{~s}^{-1}$, but the positional overlap of the narrow dust lane feature with the broad circumnuclear feature. It is worth noting that the velocity field of the inner region of the CND seems to twist in the $\mathrm{HCO}^{+}(1-0)$ moment 1 map (Figure 5). This warping appears to decrease the position angle of the inner $\mathrm{CND}$, consistent with the $\mathrm{H}_{2}$ velocity map of Neumayer et al. (2007) and CO maps of Espada et al. (2017). However, in the $\mathrm{H}_{2}$ velocity map, there appears to be another warp further in, not sampled by our data (due to the absorption).

Position-velocity (PV) diagrams for ${ }^{13} \mathrm{CO}(2-1),{ }^{13} \mathrm{CO}(1-0)$, and $\mathrm{HCO}^{+}(1-0)$ are shown in Figure 6. The top left panel of the figure shows the locations of the slices through the cube. Five slices were taken: the first through the major axis of the CND, the second through the inner peaks of the CND, the third and fourth 

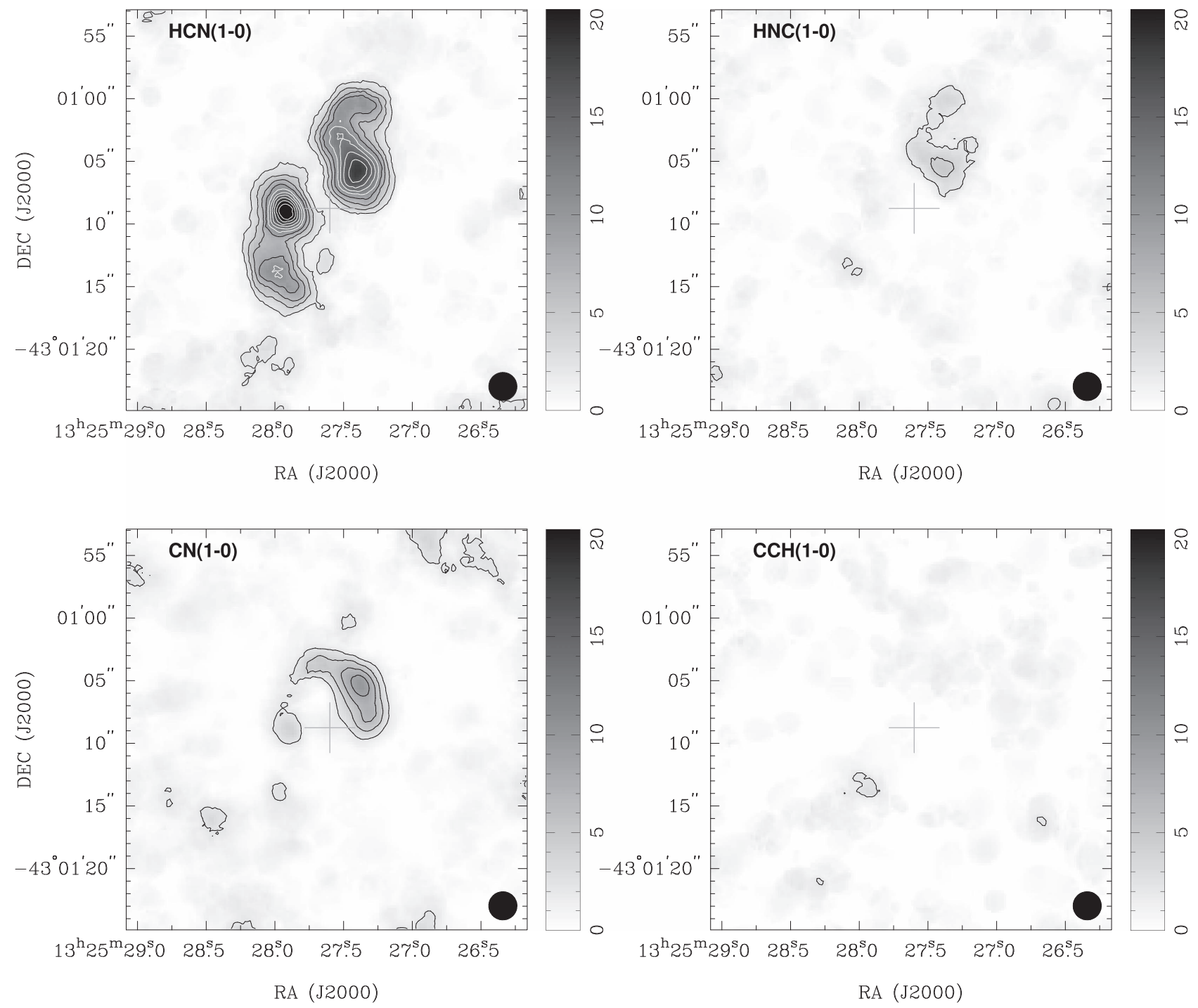

Figure 3. Complementing Figure 2, integrated intensity maps of the rest of the dense gas tracers are shown here. The top left panel displays HCN $(1-0)$, top right displays $\mathrm{HNC}(1-0)$, bottom left displays $\mathrm{CN}(1-0)$, and bottom right displays $\mathrm{CCH}(1-0)$. All of the maps are at a spatial resolution of 2 !' $25 \times 2$ !' 25 . The central cross (as in Figure 2) locates the central black hole and is scaled to $4^{\prime \prime}$ or $70 \mathrm{pc}$ at Cen A (located at R.A.: 13 $25^{\mathrm{h}} \mathrm{m}^{\mathrm{m}} 27^{\mathrm{s}} 616$ Decl.: $-43^{\circ} 01^{\prime} 08^{\prime \prime}$. 813 in J2000). All of these images share a contour spacing of $2.1 \mathrm{~K} \mathrm{~km} \mathrm{~s}^{-1}$ and are displayed on the same colorscale. This value is approximately $3 \sigma$ for each of the dense gas tracers.

along the northern and southern molecular arms, and the last along the axis of the radio jet. The slices were all averaged over a width of 11 pixels, approximately $40 \mathrm{pc}$. These pixels are not independent and were chosen such that the width of the slice was approximately the same width as the beam. The broad components in all of the PV diagrams come from the CND, while the narrow components come from the molecular arms. The center of the slice (the 0 offset location) is noted by a small cross on the slice line. Slices 1, 2, and 5 all share the same center.

Slice 1 extends along the elongated axis of the CND while also crossing the molecular arms. Slice 1 shows the lack of ${ }^{13} \mathrm{CO}(1-0)$ along with the prominence of $\mathrm{HCO}^{+}$in the CND. ${ }^{13} \mathrm{CO}(2-1)$ is present in both the molecular arm components as well as the CND components, although not as prominently as $\mathrm{HCO}^{+}$. The $\mathrm{CND}$ has components spanning a velocity range from 350 to $800 \mathrm{~km} \mathrm{~s}^{-1}$. The molecular arms span a much narrower velocity range, about $520-580 \mathrm{~km} \mathrm{~s}^{-1}$ within the band 6 primary beam in Slice 1 .
Slice 2 intersects the inner portion of the nuclear disk and avoids the molecular arms until large offsets. Again, there is a lack of ${ }^{13} \mathrm{CO}(1-0)$ in the broad (CND) components. ${ }^{13} \mathrm{CO}(2-1)$ is present in both the molecular arms as well as the CND, although more prominent in the molecular arms. This slice shows that the inner portion of the CND has a velocity range of $375-700 \mathrm{~km} \mathrm{~s}^{-1}$ while both molecular arms span velocities $\sim 475-575 \mathrm{~km} \mathrm{~s}^{-1}$.

Slices 3 and 4 primarily cover the northern and southern molecular arms, crossing only the very edges of the CND. Both slices show the narrow velocity range of the molecular arms, but slice 4 (southern arm) is simply a linear feature in the PV diagram. Slice 3 has a more complicated structure. Slice 3 (northern arm) contains a linear feature similar to slice 4 with the addition of ${ }^{13} \mathrm{CO}(1-0)$ and ${ }^{13} \mathrm{CO}(2-1)$ detected in the same position as the linear feature, but offset by $\sim 30 \mathrm{~km} \mathrm{~s}^{-1}$ showing a double structure. This double structure might be a result of overlapping tilted ring along the line of sight, but its 


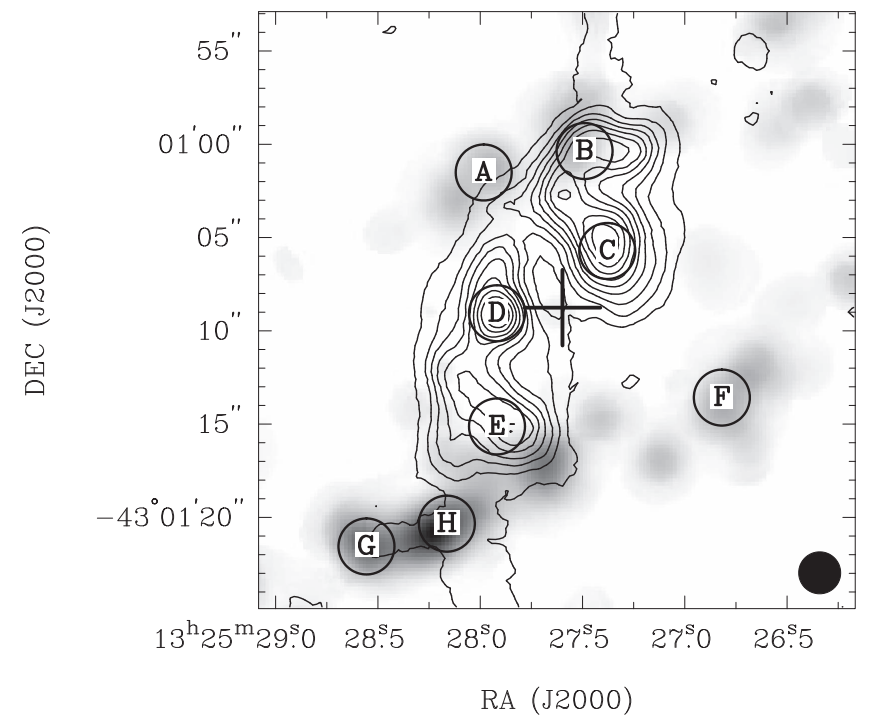

Figure 4. Schematic image of the nuclear region of Cen A. The grayscale in this figure is the integrated intensity map of ${ }^{13} \mathrm{CO}(2-1)$, and the contours correspond to the integrated intensity of $\mathrm{HCO}^{+}(1-0)$. The cross locates the central black hole, and the lengths of the cross correspond to $4^{\prime \prime}$ or $70 \mathrm{pc}$ (located at R.A. $13^{\mathrm{h}} 25^{\mathrm{m}} 27^{\mathrm{s}} .616$ Decl.: $-43^{\circ} 01^{\prime} 08^{\prime \prime} .813$ in J2000). The filled circle in the bottom right corner of the figure corresponds to the synthesized beam of $2^{\prime \prime} 25 \times 2$." 25 common to ${ }^{13} \mathrm{CO}(2-1)$ and $\mathrm{HCO}^{+}(1-0)$. The circles labeled $\mathrm{A}$ through $\mathrm{H}$ are regions of further study. Regions $\mathrm{A}, \mathrm{F}, \mathrm{G}$, and $\mathrm{H}$ are primarily probing the molecular arms, while regions $\mathrm{C}, \mathrm{D}$, and $\mathrm{E}$ probe the CND. Region B probes an overlapping region of the molecular arms and the CND.

origin is not entirely evident. The absence of emission from ${ }^{13} \mathrm{CO}(2-1)$ at an offset of $-14^{\prime \prime}$ to -20 " results from being outside the $20 \%$ level of the primary beam.

Slice 5 traces approximately the AGN jet axis. However, there is no indication of significant structure that would come from an interaction of the molecular gas with the jet.

\subsection{Spectra}

\subsubsection{Emission Spectra}

Figures 7-14 show the spectra from each of the detected transitions as well as ${ }^{12} \mathrm{CO}(2-1)$ from the science verification data and the non-detection of $\mathrm{C}^{18} \mathrm{O}(1-0)$ toward the eight marked locations in Figure 4. Spectra were taken using a 2!" 25 apertures corresponding to the circles in Figure 4. We identify 22 velocity components throughout all of the observed molecular transitions and spatial locations. Each of these components are labeled by its spatial location and a numeric identifier that increases with velocity, irrespective of the transition. These components are identified in the top left panel in Figures 7 through 14 (the ${ }^{12} \mathrm{CO}(2-1)$ spectra from each of the regions). Each figure shows the spectra from each of the detected transitions, as well as ${ }^{12} \mathrm{CO}(2-1)$ from the science verification data and the non-detection of $\mathrm{C}^{18} \mathrm{O}(1-0)$. The properties of these spectral features-that is, for each velocity component (e.g., A-1 , A-2, A-3)-are given in Table 2, and corresponding line ratios are given in Table 3. For undetected features, a 1 sigma upper limit for the peak temperature is provided. Comparisons between detections and non-detections in Table 3 are presented as upper or lower limits of that line ratio. Comparisons between double non-detections are not presented in Table 3.
Regions A, F, G, and $\mathrm{H}$ represent the molecular arm components. These regions typically contain a couple of features that have narrow line widths on the order of $10-15 \mathrm{~km}$ $\mathrm{s}^{-1}$. These features are prominent in $\mathrm{CO}$ and its isotopologues, but nearly non-existent in the dense gas tracers. $\mathrm{HCO}^{+}(1-0)$ and $\operatorname{HCN}(1-0)$ show faint narrow features in regions $\mathrm{B}, \mathrm{G}$, and $\mathrm{H}$. In regions $\mathrm{B}$ and $\mathrm{G}, \mathrm{HCO}^{+}(1-0)$ and $\mathrm{HCN}(1-0)$ are at about $1 / 2$ and $1 / 4$ of the corresponding ${ }^{13} \mathrm{CO}(1-0)$ peak temperature values, and in region $\mathrm{H}$ they are about $1 / 6$ and $1 / 8$ the ${ }^{13} \mathrm{CO}(1-0)$ value. In region $\mathrm{A}$ there is a feature near $615 \mathrm{~km} \mathrm{~s}^{-1}$ present in three of the transitions that have broad line widths, comparable to features in the CND. This feature corresponds to emission from the edge of the CND contributing to region $\mathrm{A}$.

Regions C, D, and E show the emission primarily from the CND. These regions contain spectral features that are prominent in the dense gas tracers and are nearly absent in $\mathrm{CO}$. ${ }^{12} \mathrm{CO}(2-1)$ and ${ }^{13} \mathrm{CO}(2-1)$ both contain these features from the CND, but the features are not seen in ${ }^{13} \mathrm{CO}(1-0)$ or either of the $\mathrm{C}^{18} \mathrm{O}$ transitions. These features all have much broader line widths than the features on the molecular arms, with widths on the order of $50 \mathrm{~km} \mathrm{~s}^{-1}$.

Region B contains spectral features attributed to the northern molecular arm as well as the CND. This is probably best seen in the ${ }^{12} \mathrm{CO}(2-1)$ and ${ }^{13} \mathrm{CO}(2-1)$ spectra. Components B-1 and $\mathrm{B}-2$ have narrow line widths and closely resemble the features of regions $\mathrm{A}, \mathrm{F}, \mathrm{G}$, and $\mathrm{H}$, which correspond to the molecular arms. Component B-3 has a much broader line width, more similar to the features in regions $\mathrm{C}, \mathrm{D}$, and $\mathrm{E}$. Region B, therefore, shows a spatial region where the molecular arm component and the CND overlap.

\subsubsection{Absorption Spectra}

The absorption spectra (Figure 15) were made by taking a spectrum through the central point of an image cube for each transition. Each spectrum was then converted to units of optical depth $(\tau)$ based on the continuum flux from Table 1, assuming a continuum source covering factor of unity (see Muller et al. 2009, their Section 4.1, for the size scale of the continuum) and the equation $\tau=-\ln \left[1-\left(\frac{-F_{\text {spec }}}{F_{\text {cont }}}\right)\right]$, where $F_{\text {spec }}$ and $F_{\text {cont }}$ are the continuum subtracted line flux and the continuum flux, respectively. There are two groups of absorption lines detected in the various molecular lines. There is one group near the systemic velocity of Cen A $\left(550 \mathrm{~km} \mathrm{~s}^{-1}\right.$; Section 3.3) that has three main components within $10 \mathrm{~km} \mathrm{~s}^{-1}$ and multiple blended or weaker components called the low velocity complex (LV complex). The other group is redshifted by $20-70 \mathrm{~km} \mathrm{~s}^{-1}$ and contains many blended components called the high velocity complex (HV complex). Both the low and high velocity complexes were previously detected in $\mathrm{HCO}^{+}(1-0), \mathrm{HCN}(1-0)$, and $\mathrm{HNC}(1-0)$ at a lower velocity resolution and sensitivity by Wiklind \& Combes (1997). We restrict our analysis of the absorption spectra to three of the narrow components $\left(539.7,543.3\right.$, and $549.7 \mathrm{~km} \mathrm{~s}^{-1}$ ) in the LV complex and a median value taken from $576-604 \mathrm{~km} \mathrm{~s}^{-1}$ of the $\mathrm{HV}$ complex and only include the molecular transitions $\mathrm{C}^{18} \mathrm{O}(1-0)$ (where no emission was detected) and those also detected in emission. 

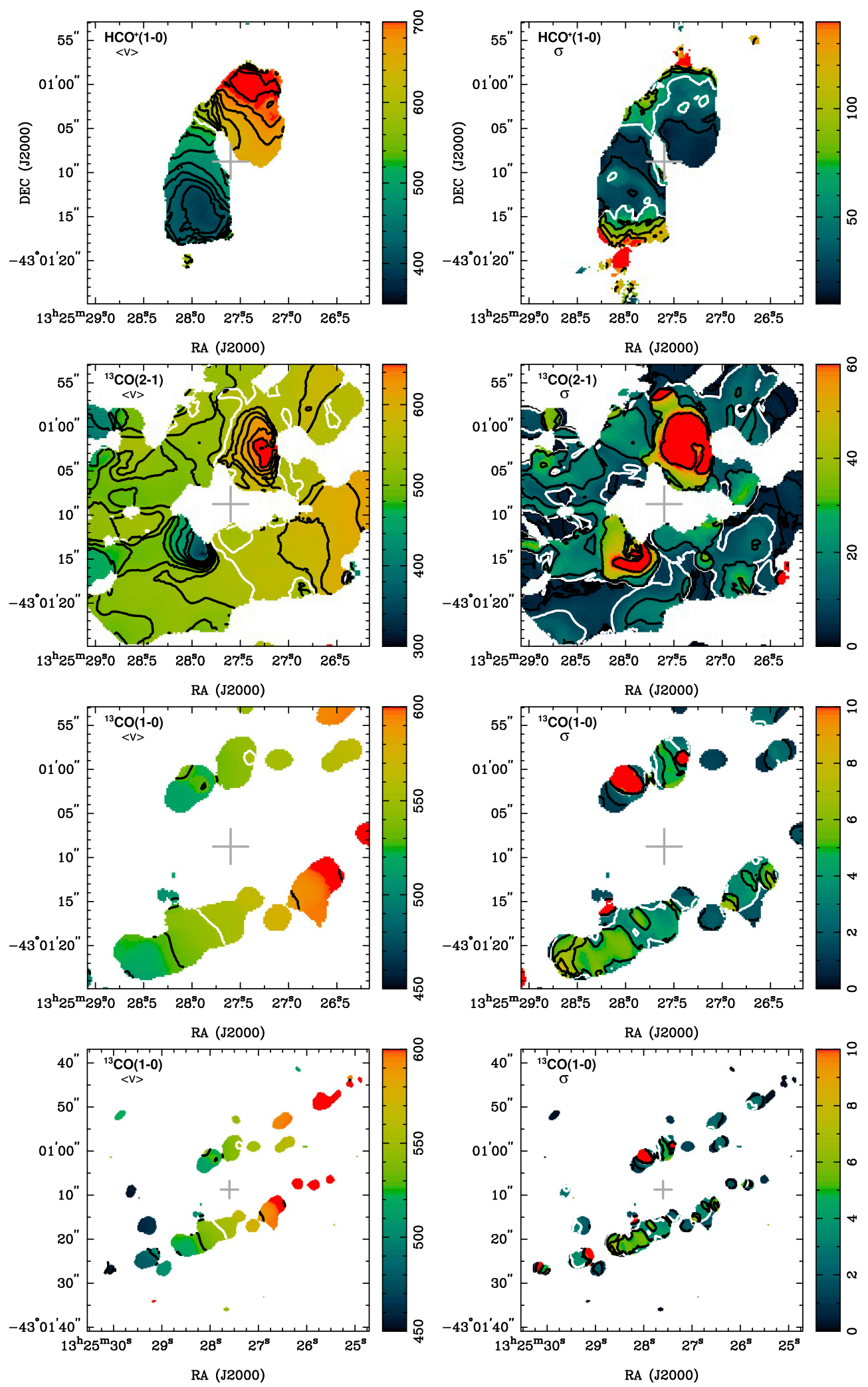

Figure 5. Intensity-weighted velocity field (moment 1) (left) and intensity-weighted velocity dispersion (moment 2$)$ (right) are shown here for $\mathrm{HCO}^{+}(1-0)$, ${ }^{13} \mathrm{CO}(2-1)$, and ${ }^{13} \mathrm{CO}(2-1)$. The last image in each column is a full field view of the ${ }^{13} \mathrm{CO}(1-0)$ moment 1 map showing the entire $3 \mathrm{~mm}$ primary beam. Each of the moment 1 maps has a contour spacing of $20 \mathrm{~km} \mathrm{~s}^{-1}$. The white contour in each of these images corresponds to a velocity of $550 \mathrm{~km} \mathrm{~s}^{-1}$. The $\mathrm{HCO}^{+}(1-0) \mathrm{moment}^{-}$ 2 map has a contour spacing of $25 \mathrm{~km} \mathrm{~s}^{-1}$, and the white contour marks a velocity dispersion of $50 \mathrm{~km} \mathrm{~s}^{-1}$. The moment 2 map of ${ }^{13} \mathrm{CO}(2-1)$ has contour spacing of $2^{n} \mathrm{~km} \mathrm{~s}^{-1}$ with $n=1,2,3,4,5,6$ (chosen to show the wide range of velocity dispersion). The white contour in this image is at $8 \mathrm{~km} \mathrm{~s}{ }^{-1}$. The ${ }^{13} \mathrm{CO}(1-0)$ moment 2 map has a contour spacing of $1.5 \mathrm{~km} \mathrm{~s}^{-1}$, with the white contour marking $3 \mathrm{~km} \mathrm{~s}^{-1}$. 

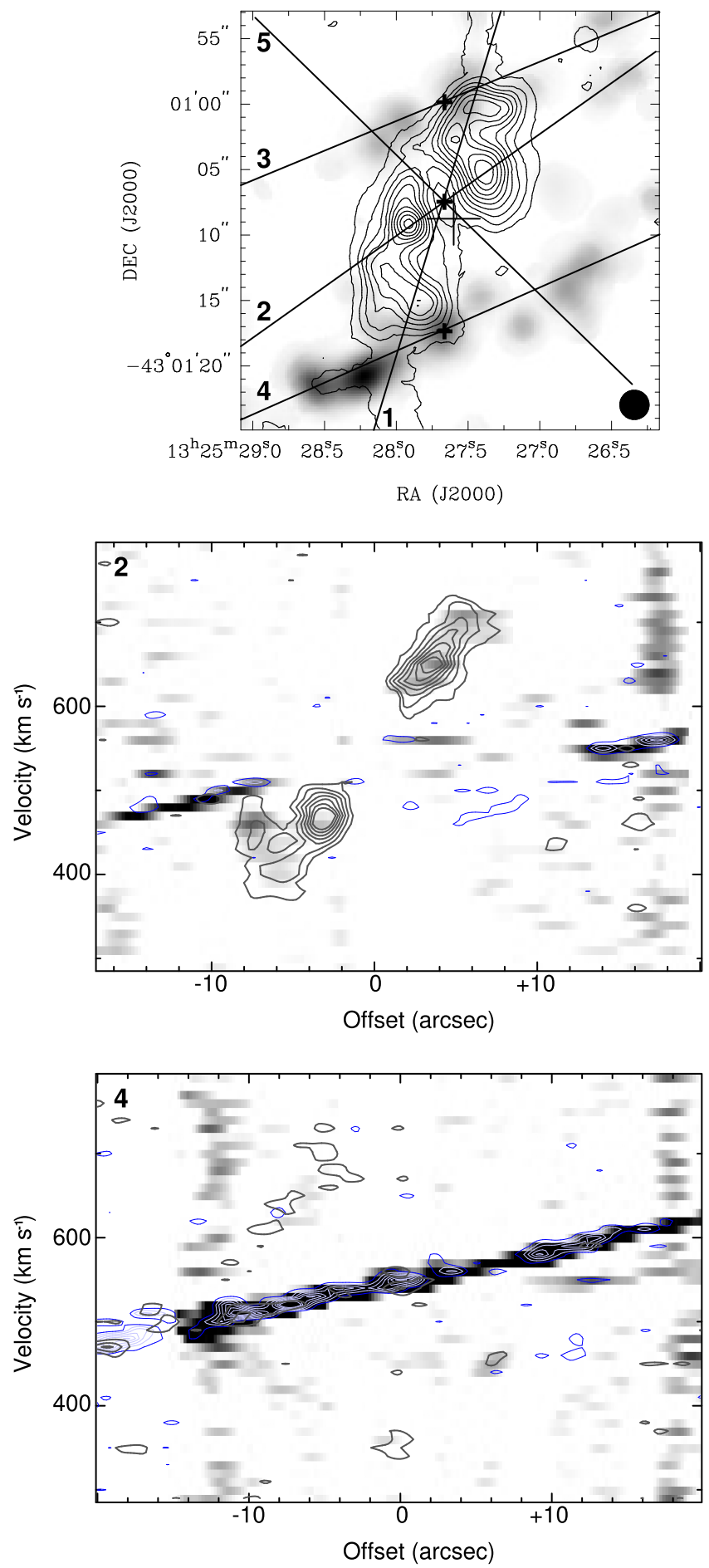
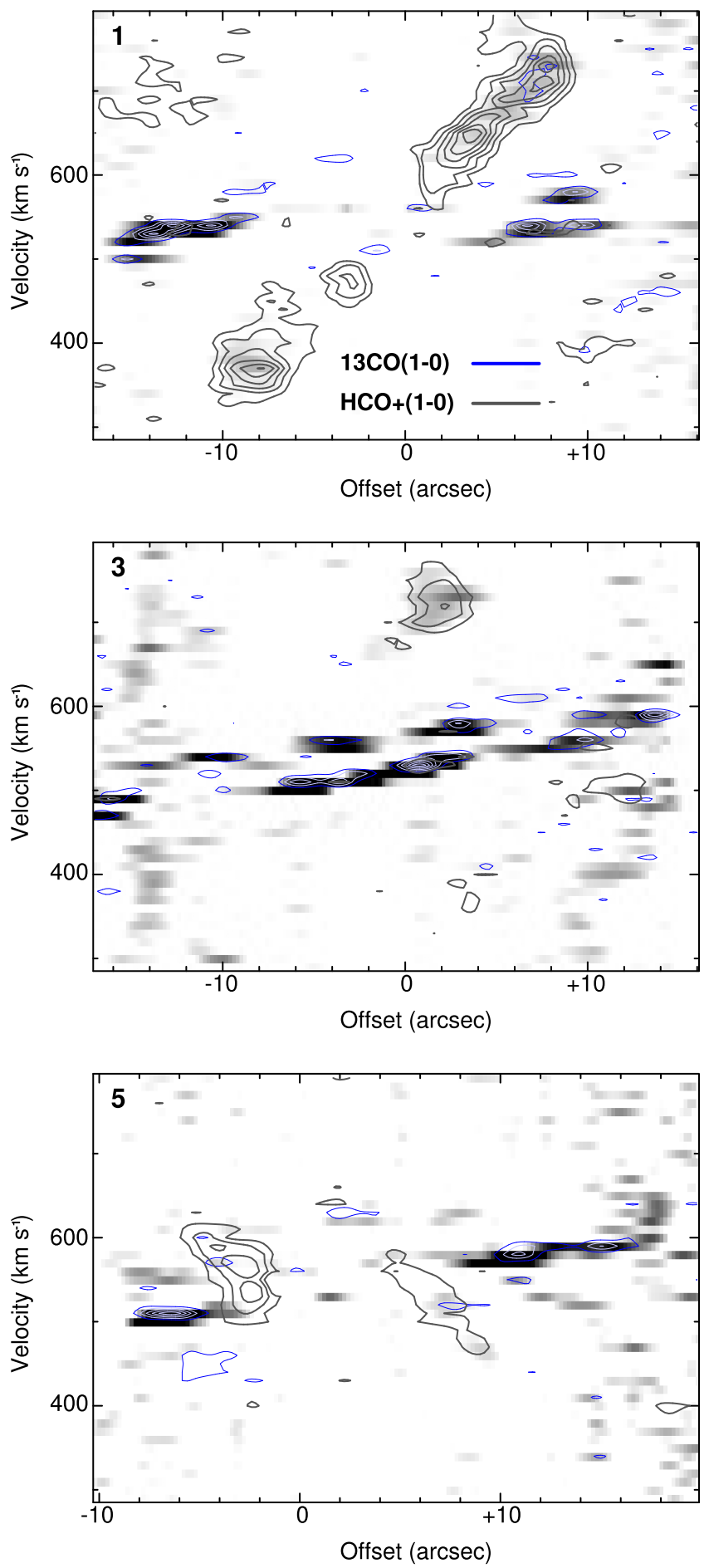

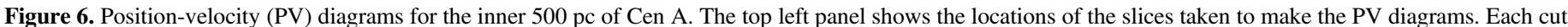

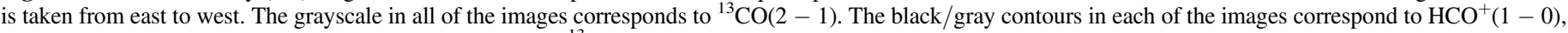

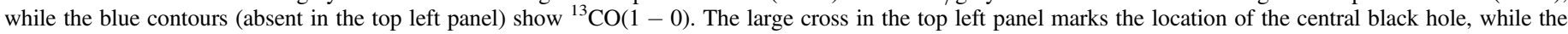
smaller black crosses mark the zero offset of each of the slices ( $1^{\prime \prime} \simeq 18$ pc offset). Slices 1,2 , and 5 all share the same central point.

\section{Discussion}

The flow of gas in the vicinity of an AGN is important in understanding the fueling of the AGN. The nuclear region of Cen A has multiple components of molecular gas, including two linear features that cross nearly in front of the AGN (see ${ }^{13} \mathrm{CO}(1-0)$ panels in Figure 6), a circumnuclear disk approximately $400 \mathrm{pc}$ in diameter, and absorption complexes both near the systemic velocity (LV) and redshifted by $20-70 \mathrm{~km} \mathrm{~s}^{-1}(\mathrm{HV})$. We investigate connections between these components both dynamically and chemically.

\subsection{Are the Molecular Arms and the CND Connecting?}

We do not detect emission connecting the narrow line width emission components of the molecular arms and the broad line width emission from the molecular CND. Region B and partially E in Figure 4 mark the locations where 

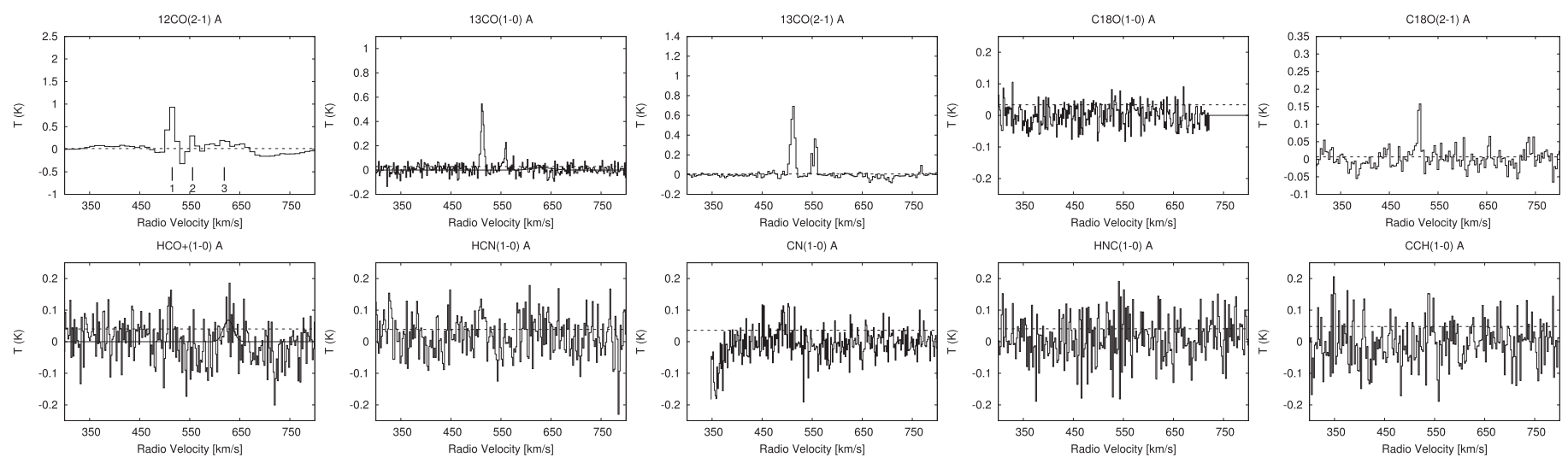

Figure 7. Emission spectra taken toward region A (see Figure 4; R.A.: $13^{\mathrm{h}} 25^{\mathrm{m}} 27^{\mathrm{s}} .983$ Decl.: $-43^{\circ} 01^{\prime} 01^{\prime \prime} .519$ in J2000). In this and Figures $8-14$, the top row shows (from left to right) ${ }^{12} \mathrm{CO}(2-1),{ }^{13} \mathrm{CO}(1-0),{ }^{13} \mathrm{CO}(2-1), \mathrm{C}^{18} \mathrm{O}(1-0)$, and $\mathrm{C}^{18} \mathrm{O}(2-1)$. The bottom row shows (from left to right) $\mathrm{HCO}(1-0), \mathrm{HCN}(1-0), \mathrm{CN}(1-0)$, $\mathrm{HNC}$ $(1-0)$, and $\mathrm{CCH}(1-0)$. The velocity axes range from 300 to $800 \mathrm{~km} \mathrm{~s}^{-1}$, and the brightness temperature axes are adjusted for each plot to highlight the emission. The dashed line on each panel of this figure shows the $1 \sigma$ noise level for that transition. For Figures 8-14, the corresponding transition will have the same $1 \sigma$ limit. For this figure as well as Figures 8-14, each detected feature that is $\leqslant 3 \sigma$ the Gaussian fit is shown as a solid line to guide the eye.
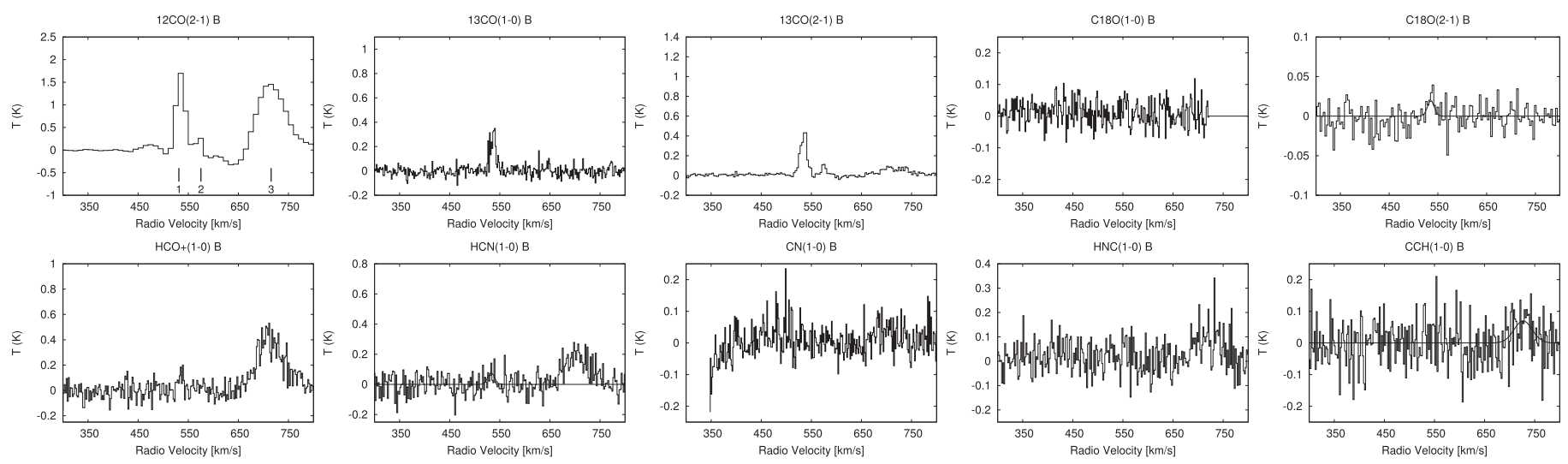

Figure 8. Emission spectra taken toward region B (see Figure 4; R.A.: $13^{\mathrm{h}} 25^{\mathrm{m}} 27^{\mathrm{s}} .490$ Decl.: $-43^{\circ} 01^{\prime} 00^{\prime \prime} 370$ in J2000). See Figure 7 for details.
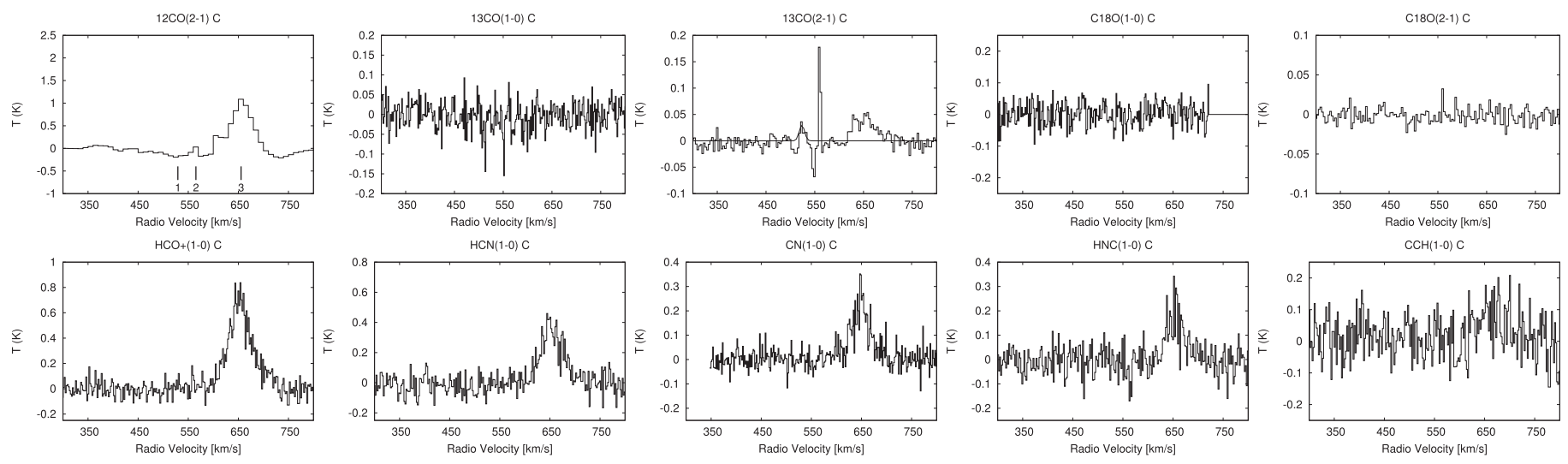

Figure 9. Emission spectra taken toward region C (see Figure 4; R.A.: $13^{\mathrm{h}} 25^{\mathrm{m}} 27^{\mathrm{s}} .379$ Decl.: $-43^{\circ} 01^{\prime} 05^{\prime \prime} \cdot 728$ in J2000). See Figure 7 for details.

the CND and the molecular arms appear to spatially overlap. According to the moment 2 maps of ${ }^{13} \mathrm{CO}(2-1)$, regions $\mathrm{B}$ and $\mathrm{E}$ correspond to high velocity dispersion regions. However, looking at the spectra taken toward these regions (see Figures 8 and 11), the cause of the high dispersion is multiple separate kinematic components along the lines of sight. In region B there is a weak broad component and two narrow components that are kinematically distinct. In region $\mathrm{E}$ there is a single narrow component and a kinematically distinct broad component. In the PV diagrams for slices 1 and 2 (Figure 6), there does not appear to be any connection between the broad, high velocity gradient component, corresponding to the CND, and the narrow, low velocity gradient component, corresponding to the molecular arms. However, due to the missing short spacings of the observations, there may be large scale structure that is resolved out, possibly hiding the connection described in Espada et al. (2009). An absence of an observed connection 

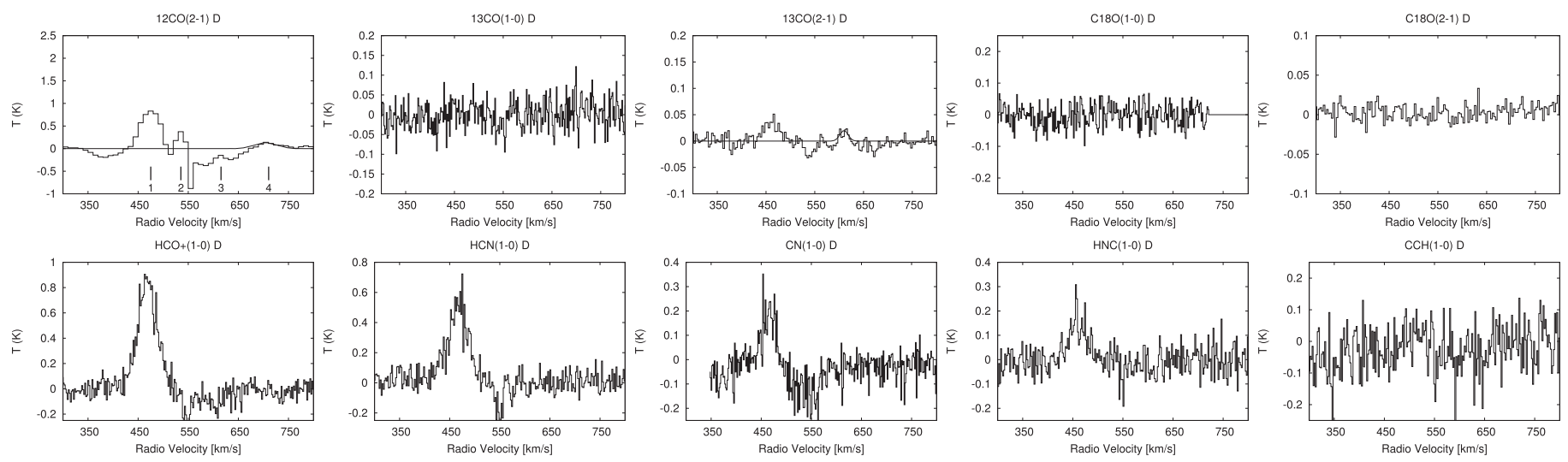

Figure 10. Emission spectra taken toward region D (see Figure 4; R.A.: $13^{\mathrm{h}} 25^{\mathrm{m}} 27^{\mathrm{s}} 918$ Decl.: $-43^{\circ} 01^{\prime} 09^{\prime \prime}$. 025 in J2000). Being near the AGN, there are absorption artifacts introduced during the continuum subtraction. See Figure 7 for details.
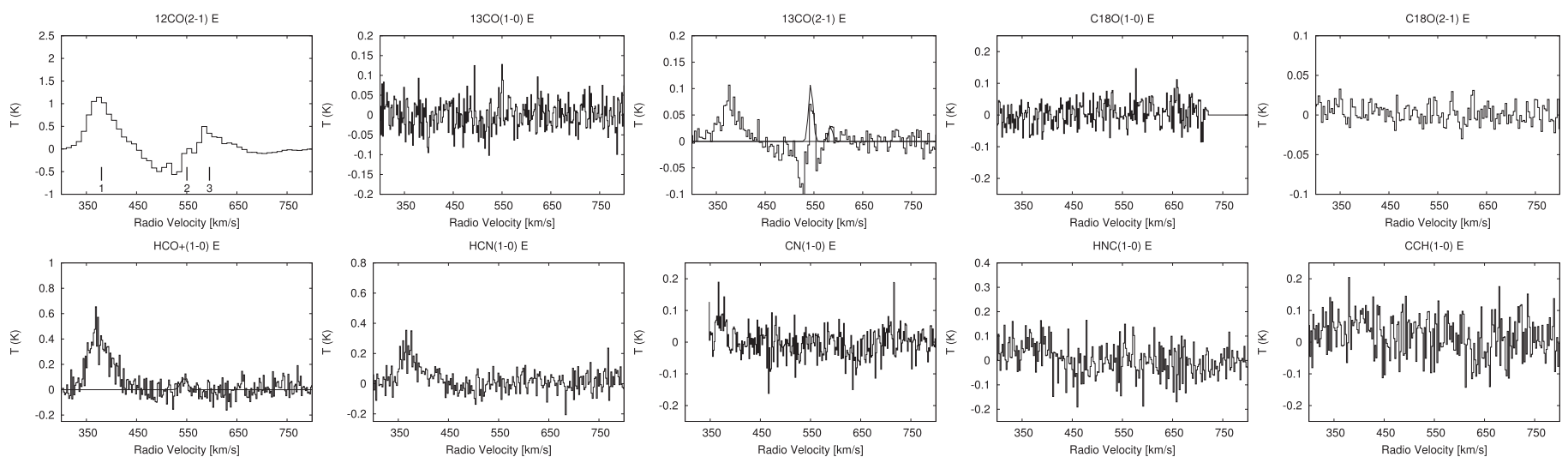

Figure 11. Emission spectra taken toward region E (see Figure 4; R.A.: $13^{\mathrm{h}} 25^{\mathrm{m}} 27^{\mathrm{s}} .918$ Decl.: $-43^{\circ} 01^{\prime} 15^{\prime \prime}$. 132 in J2000). Being near the AGN, there are absorption artifacts introduced during the continuum subtraction. See Figure 7 for details.

between the two main emission components means the method for transporting gas from the molecular arms to the CND is still not clear.

\subsection{Gas Chemistry}

\subsubsection{CO Isotopic Ratios}

Intensity ratios between isotopologue transitions with the same J states, I[ $\left.{ }^{12} \mathrm{CO}(2-1)\right] / \mathrm{I}\left[{ }^{13} \mathrm{CO}(2-1)\right], \mathrm{I}\left[{ }^{12} \mathrm{CO}(2-1)\right] / \mathrm{I}$ $\left[\mathrm{C}^{18} \mathrm{O}(2-1)\right]$, and $\mathrm{I}\left[{ }^{13} \mathrm{CO}(2-1)\right] / \mathrm{I}\left[\mathrm{C}^{18} \mathrm{O}(2-1)\right]$, contain information on the opacities and abundance ratios of $\mathrm{CO}$ and its isotopologues, assuming each pair of transitions has a common excitation temperature $T_{x}$. For example, the intensity ratio, $\mathrm{I}\left[{ }^{12} \mathrm{CO}(2-1)\right] / \mathrm{I}\left[{ }^{13} \mathrm{CO}(2-1)\right]$, with the same line width for 2-1 and 1-0 and in the absence of fractionation or selective photodissociation, gives

$$
\frac{{ }^{12} \mathrm{~T}_{21}}{{ }^{13} \mathrm{~T}_{21}} \simeq \frac{{ }^{12} f_{a}\left({ }^{12} J_{21}\left(T_{x}\right)-{ }^{12} J_{21}\left(T_{b g}\right)\right)\left(1-e^{-{ }^{12} \tau}\right)}{{ }^{13} f_{a}\left({ }^{13} J_{21}\left(T_{x}\right)-{ }^{13} J_{21}\left(I_{b g}\right)\right)\left(1-e^{-A^{12} \tau}\right)},
$$

with ${ }^{13} \tau$ replaced by $A^{12} \tau$, where $\mathrm{A}$ is the isotopic abundance ratio $\left[{ }^{12} \mathrm{CO} /{ }^{13} \mathrm{CO}\right]$, and with

$$
J_{\nu}(T)=\frac{\frac{\mathrm{h} \nu}{\mathrm{k}}}{e^{\frac{\mathrm{h} \nu}{\mathrm{k} T_{\mathrm{x}}}}-1} .
$$

In the high temperature limit, ${ }^{12} J_{i j}\left(T_{i j}\right)$ simply becomes $T_{i j}$, and assuming similar area filling factors, ${ }^{i} f_{a}$, and the same $T_{x}$, this can be simplified to ${ }^{12} \mathrm{I}_{21} /{ }^{13} \mathrm{I}_{21}=\left(1-e^{-{ }^{12} \tau}\right) /\left(1-e^{-A^{12} \tau}\right)$ (e.g., Aalto et al. 1995).

Similarly, taking ratios of the rotational transitions of ${ }^{13} \mathrm{CO}$ (and $\mathrm{C}^{18} \mathrm{O}$ ), between different $\mathrm{J}$ states of the same isotopologue like $\mathrm{I}\left[{ }^{13} \mathrm{CO}(2-1)\right] / \mathrm{I}\left[{ }^{13} \mathrm{CO}(1-0)\right]$, constrains $T_{x}$. Again, assuming high temperature limit and identical line widths and filling factors, the line temperature ratio is approximated by the equation

$$
\frac{{ }^{13} T_{\mathrm{mb}}^{21}}{{ }^{13} T_{\mathrm{mb}}^{10}} \simeq \frac{\left(T_{21}-T_{21}^{\mathrm{bg}}\right)\left(1-e^{-{ }^{13}} \tau_{21}\right)}{\left(T_{10}-T_{10}^{\mathrm{bg}}\right)\left(1-e^{-{ }^{13}} \tau_{10}\right)} .
$$

In the optically thick limit, this becomes

$$
\frac{{ }^{13} T_{\mathrm{mb}}^{21}}{{ }^{13} T_{\mathrm{mb}}^{10}} \simeq \frac{\left(T_{21}-T_{21}^{\mathrm{bg}}\right)}{\left(T_{10}-T_{10}^{\mathrm{bg}}\right)} \simeq 1,
$$

and in the optically thin limit, we obtain

$$
\frac{{ }^{13} T_{\mathrm{mb}}^{21}}{{ }^{13} T_{\mathrm{mb}}^{10}} \simeq \frac{\tau_{21}\left(T_{21}-T_{21}^{\mathrm{bg}}\right)}{\tau_{10}\left(T_{10}-T_{10}^{\mathrm{bg}}\right)} .
$$

The emission ratios between isotopologue transitions with the same $\mathbf{J}$ states do not give unique values for the opacities and the abundance ratios separately, so in order to get values to compare, we assume that the $\left[{ }^{12} \mathrm{CO} /{ }^{13} \mathrm{CO}\right]$ abundance ratio is near 40, the values found toward the centers of NGC 253 (Henkel et al. 2014) and IC 342 (Meier \& Turner 2000). We observe an emission line ratio ${ }^{13} \mathrm{CO}(2-1) /{ }^{13} \mathrm{CO}(1-0)$ of 

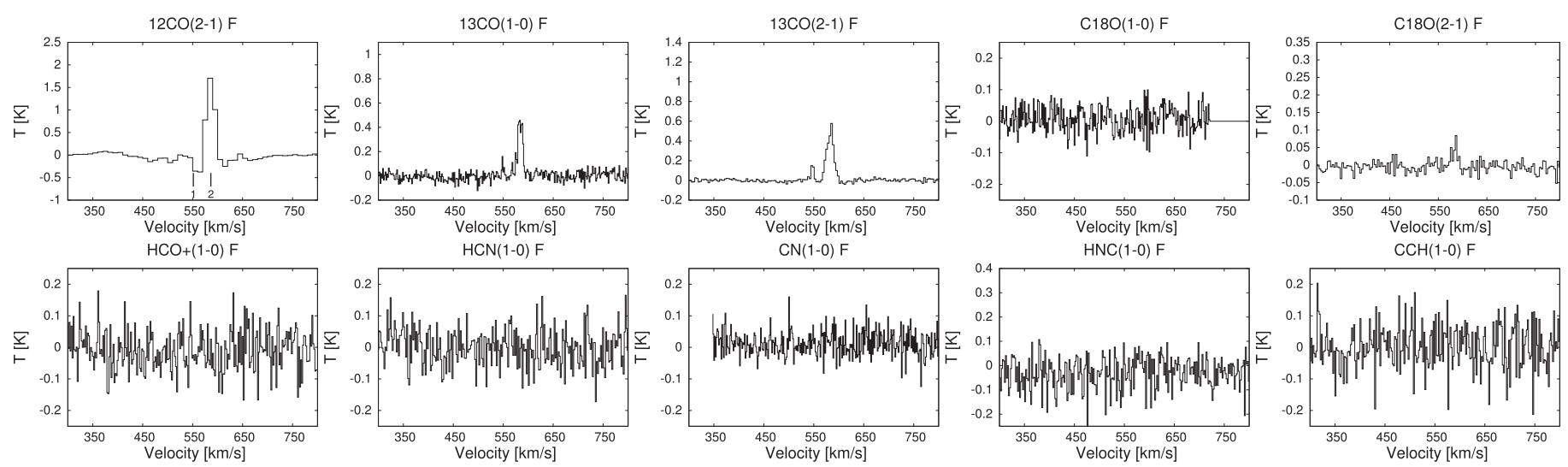

Figure 12. Emission spectra taken toward region F (see Figure 4; R.A.: $13^{\mathrm{h}} 25^{\mathrm{m}} 26^{\mathrm{s}} .819$ Decl.: $-43^{\circ} 01^{\prime} 13^{\prime \prime} 585$ in J2000). See Figure 7 for details.
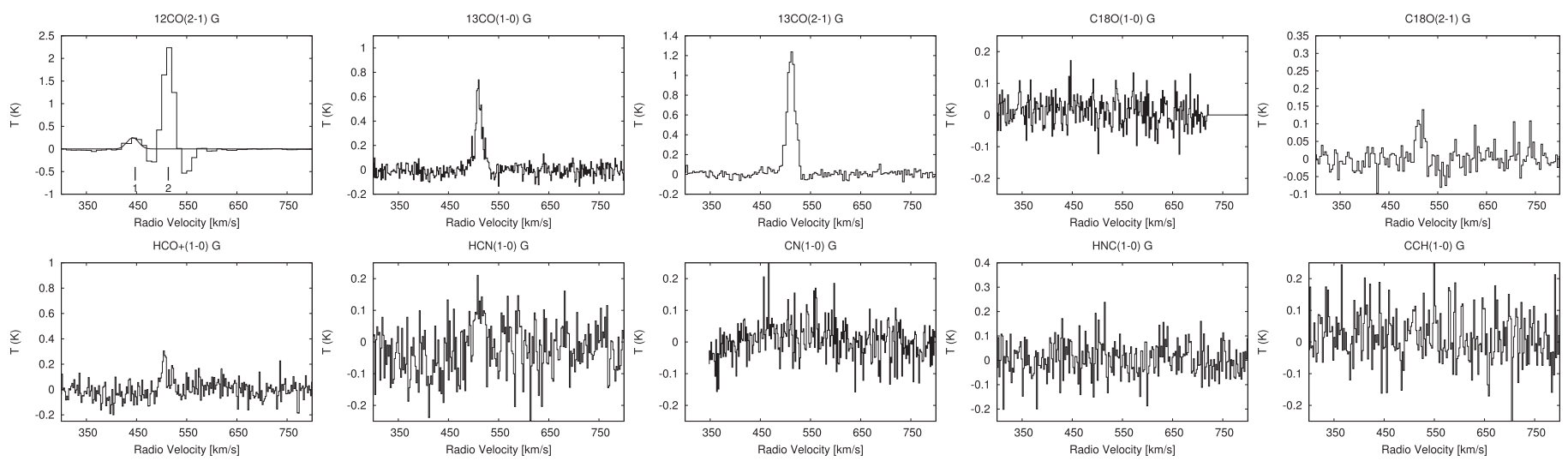

Figure 13. Emission spectra taken toward region G (see Figure 4; R.A.: $13^{\mathrm{h}} 25^{\mathrm{m}} 28^{\mathrm{s}} .556$ Decl.: $-43^{\circ} 01^{\prime} 21^{\prime \prime}$. 565 in J2000). See Figure 7 for details.
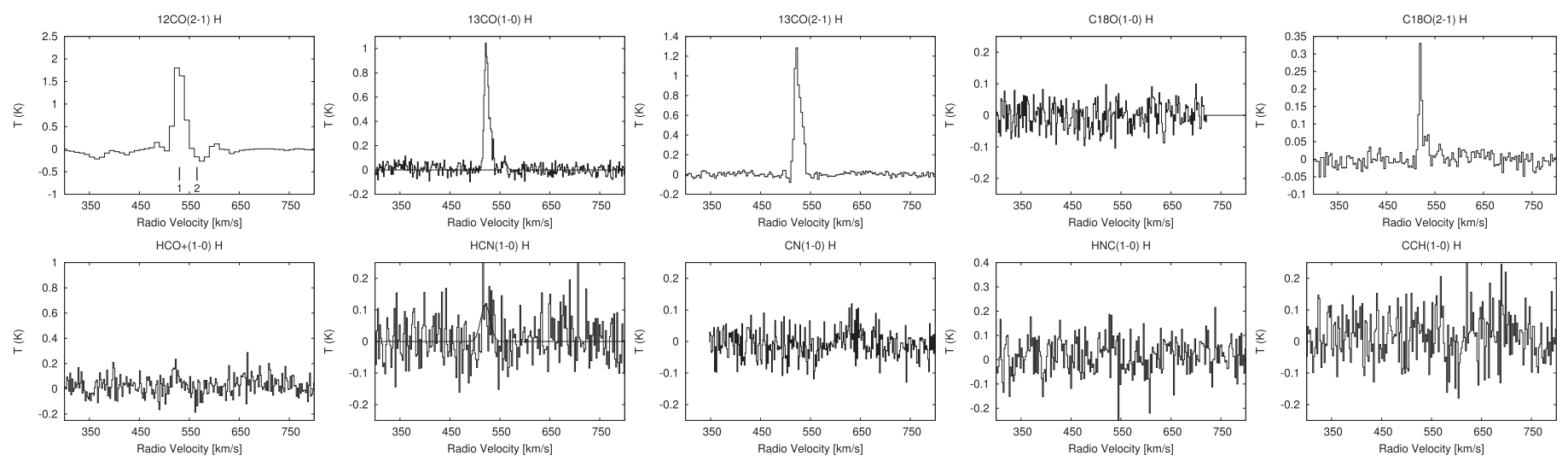

Figure 14. Emission spectra taken toward region H (see Figure 4; R.A.: $13^{\mathrm{h}} 25^{\mathrm{m}} 28^{\mathrm{s}} .556$ Decl.: $-43^{\circ} 01^{\prime} 20^{\prime \prime} .343$ in J2000). See Figure 7 for details.

1.1-1.5. The emission ratios are consistent with the single dish data from Israel et al. (2014).

We discuss five spectral features along the molecular arms that contain detections of ${ }^{12} \mathrm{CO}(2-1),{ }^{13} \mathrm{CO}(2-1)$, $\mathrm{C}^{18} \mathrm{O}(2-1)$, and ${ }^{13} \mathrm{CO}(1-0)$ : $\mathrm{A}-1, \mathrm{~B}-1, \mathrm{~F}-2, \mathrm{G}-2$, and $\mathrm{H}-1$. By starting near an abundance ratio $\left[{ }^{12} \mathrm{C} /{ }^{13} \mathrm{C}\right]=40$, we adjust the abundance ratio and opacity for the isotopologue ratios until a consistent solution is found with the observed intensity ratios. We find best fit values for the abundance ratios for features $\mathrm{A}-1$ and $\mathrm{H}-1$ of $\left[{ }^{12} \mathrm{CO} /{ }^{13} \mathrm{CO}\right]=50$, slightly raised from the value near the center of NGC 253 and IC 342 and $\left[{ }^{16} \mathrm{O} /{ }^{18} \mathrm{O}\right]$ of about 300 . The $\left[{ }^{16} \mathrm{O} /{ }^{18} \mathrm{O}\right]$ abundance ratio for the nucleus of NGC 253 was found to be $\sim 200$ by Henkel et al. (1993), which is consistent with the inner Galaxy (Wilson \& Rood 1994). Opacities for ${ }^{12} \mathrm{CO}(2-1)$ of around 50 and for ${ }^{13} \mathrm{CO}(2-1)$ of around unity are implied for both A-1 and $\mathrm{H}-1$. Spectral feature $\mathrm{F}-2$ requires the $\left[{ }^{16} \mathrm{O} /{ }^{18} \mathrm{O}\right]$ abundance ratio to change from $\left[{ }^{16} \mathrm{O} /{ }^{18} \mathrm{O}\right]=300$ to $\left[{ }^{16} \mathrm{O} /{ }^{18} \mathrm{O}\right]=350$ to maintain agreement with the $\left[{ }^{12} \mathrm{CO} /{ }^{13} \mathrm{CO}\right]$ abundance. For F-2, ${ }^{12} \mathrm{CO}$ opacities of around 15 are implied. The isotopic ratios for spectral features B- 1 and G-2 exhibit more extreme values. To match the ratios with $\left[{ }^{12} \mathrm{CO} /{ }^{13} \mathrm{CO}\right]$ around $40,\left[{ }^{16} \mathrm{O} /{ }^{18} \mathrm{O}\right]$ would be pushed to $>800$, while keeping $\left[{ }^{16} \mathrm{O} /{ }^{18} \mathrm{O}\right]$ around 300 would require $\left[{ }^{12} \mathrm{CO} /{ }^{13} \mathrm{CO}\right]$ to drop to $<20$. Opacities for 
Table 2

Properties of Emission Components

\begin{tabular}{|c|c|c|c|c|c|}
\hline Line Identifier & Transition & $\begin{array}{l}\text { Peak } \\
(\mathrm{K})\end{array}$ & $\begin{array}{l}\text { Line Center } \\
\left(\mathrm{km} \mathrm{s}^{-1}\right)\end{array}$ & $\begin{array}{l}\text { FWHM } \\
\left(\mathrm{km} \mathrm{s}^{-1}\right)\end{array}$ & $\begin{array}{c}\text { Integral } \\
\left(\mathrm{K} \mathrm{km} \mathrm{s}^{-1}\right)\end{array}$ \\
\hline A-1 & ${ }^{12} \mathrm{CO}(2-1)$ & $0.92 \pm 0.12$ & $510.0 \pm 5.0$ & $20.0 \pm 5.0$ & $19.4 \pm 5.6$ \\
\hline$(13: 25: 27.983)$ & ${ }^{13} \mathrm{CO}(2-1)$ & $0.61 \pm 0.03$ & $508.5 \pm 0.3$ & $10.4 \pm 0.6$ & $6.7 \pm 0.5$ \\
\hline \multirow[t]{8}{*}{$(-43.01 .01 .519)$} & ${ }^{13} \mathrm{CO}(1-0)$ & $0.42 \pm 0.03$ & $513.1 \pm 0.3$ & $7.8 \pm 0.8$ & $3.5 \pm 0.4$ \\
\hline & $\mathrm{C}^{18} \mathrm{O}(2-1)$ & $0.14 \pm 0.02$ & $509.6 \pm 0.6$ & $8.5 \pm 1.4$ & $1.3 \pm 0.3$ \\
\hline & $\mathrm{C}^{18} \mathrm{O}(1-0)$ & $<0.03$ & ... & $\ldots$ & $\ldots$ \\
\hline & $\mathrm{HCO}^{+}(1-0)$ & $<0.06$ & $\cdots$ & $\cdots$ & $\cdots$ \\
\hline & $\mathrm{HCN}(1-0)$ & $<0.06$ & $\ldots$ & $\ldots$ & $\ldots$ \\
\hline & $\mathrm{CN}(1-0)$ & $<0.05$ & $\ldots$ & $\ldots$ & $\ldots$ \\
\hline & $\mathrm{HNC}(1-0)$ & $<0.06$ & $\ldots$ & $\ldots$ & $\ldots$ \\
\hline & $\mathrm{CCH}(1-0)$ & $<0.06$ & $\ldots$ & $\ldots$ & $\ldots$ \\
\hline \multirow[t]{10}{*}{ A-2 } & ${ }^{12} \mathrm{CO}(2-1)$ & $0.31 \pm 0.05$ & $550.0 \pm 5.0$ & $15.0 \pm 5.0$ & $4.9 \pm 3.6$ \\
\hline & ${ }^{13} \mathrm{CO}(2-1)$ & $0.26 \pm 0.07$ & $553.7 \pm 1.3$ & $10.8 \pm 3.2$ & $3.0 \pm 1.2$ \\
\hline & ${ }^{13} \mathrm{CO}(1-0)$ & $0.18 \pm 0.01$ & $560.1 \pm 0.4$ & $4.7 \pm 0.9$ & $0.9 \pm 0.2$ \\
\hline & $\mathrm{C}^{18} \mathrm{O}(2-1)$ & $<0.02$ & $\ldots$ & $\ldots$ & $\ldots$ \\
\hline & $\mathrm{C}^{18} \mathrm{O}(1-0)$ & $<0.03$ & $\cdots$ & $\cdots$ & $\cdots$ \\
\hline & $\mathrm{HCO}^{+}(1-0)$ & $<0.06$ & $\ldots$ & $\ldots$ & $\cdots$ \\
\hline & $\mathrm{HCN}(1-0)$ & $<0.06$ & $\cdots$ & $\cdots$ & $\cdots$ \\
\hline & $\mathrm{CN}(1-0)$ & $<0.05$ & $\cdots$ & $\cdots$ & $\cdots$ \\
\hline & $\mathrm{HNC}(1-0)$ & $<0.06$ & $\cdots$ & $\cdots$ & $\ldots$ \\
\hline & $\mathrm{CCH}(1-0)$ & $<0.06$ & $\ldots$ & $\ldots$ & $\ldots$ \\
\hline \multirow[t]{10}{*}{ A-3 } & ${ }^{12} \mathrm{CO}(2-1)$ & $0.15 \pm 0.03$ & $614.1 \pm 6.3$ & $61.0 \pm 15.0$ & $10.0 \pm 3.3$ \\
\hline & ${ }^{13} \mathrm{CO}(2-1)$ & $<0.02$ & $\ldots$ & $\ldots$ & $\ldots$ \\
\hline & ${ }^{13} \mathrm{CO}(1-0)$ & $0.02 \pm 0.01$ & $631.8 \pm 12.0$ & $40.0 \pm 31.0$ & $0.9 \pm 0.8$ \\
\hline & $\mathrm{C}^{18} \mathrm{O}(2-1)$ & $<0.02$ & $\ldots$ & $\ldots$ & $\ldots$ \\
\hline & $\mathrm{C}^{18} \mathrm{O}(1-0)$ & $<0.03$ & $\cdots$ & $\cdots$ & $\cdots$ \\
\hline & $\mathrm{HCO}^{+}(1-0)$ & $0.07 \pm 0.03$ & $627.1 \pm 3.8$ & $19.6 \pm 9.0$ & $1.5 \pm 0.9$ \\
\hline & $\mathrm{HCN}(1-0)$ & $<0.06$ & $\ldots$ & $\ldots$ & $\ldots$ \\
\hline & $\mathrm{CN}(1-0)$ & $<0.05$ & $\cdots$ & $\cdots$ & $\cdots$ \\
\hline & $\mathrm{HNC}(1-0)$ & $<0.06$ & $\cdots$ & $\cdots$ & $\cdots$ \\
\hline & $\mathrm{CCH}(1-0)$ & $<0.06$ & $\ldots$ & $\cdots$ & $\cdots$ \\
\hline B-1 & ${ }^{12} \mathrm{CO}(2-1)$ & $2.17 \pm 0.05$ & $525.9 \pm 0.2$ & $22.8 \pm 0.6$ & $52.5 \pm 1.7$ \\
\hline$(13: 25: 27.490)$ & ${ }^{13} \mathrm{CO}(2-1)$ & $0.42 \pm 0.02$ & $533.1 \pm 0.4$ & $16.0 \pm 0.9$ & $7.2 \pm 0.5$ \\
\hline \multirow[t]{8}{*}{$(-43.01 .00 .370)$} & ${ }^{13} \mathrm{CO}(1-0)$ & $0.29 \pm 0.04$ & $537.6 \pm 0.9$ & $13.8 \pm 2.0$ & $4.3 \pm 0.8$ \\
\hline & $\mathrm{C}^{18} \mathrm{O}(2-1)$ & $0.02 \pm 0.01$ & $535.5 \pm 2.2$ & $13.5 \pm 5.1$ & $0.5 \pm 0.2$ \\
\hline & $\mathrm{C}^{18} \mathrm{O}(1-0)$ & $<0.04$ & $\ldots$ & $\ldots$ & $\ldots$ \\
\hline & $\mathrm{HCO}^{+}(1-0)$ & $0.14 \pm 0.03$ & $534.4 \pm 1.3$ & $11.1 \pm 3.1$ & $1.7 \pm 0.6$ \\
\hline & $\mathrm{HCN}(1-0)$ & $0.08 \pm 0.03$ & $532.7 \pm 2.3$ & $12.9 \pm 5.5$ & $1.1 \pm 0.6$ \\
\hline & $\mathrm{CN}(1-0)$ & $<0.05$ & $\ldots$ & $\ldots$ & $\ldots$ \\
\hline & $\mathrm{HNC}(1-0)$ & $<0.06$ & $\cdots$ & $\cdots$ & $\cdots$ \\
\hline & $\mathrm{CCH}(1-0)$ & $<0.06$ & $\ldots$ & $\ldots$ & $\ldots$ \\
\hline \multirow[t]{10}{*}{ B-2 } & ${ }^{12} \mathrm{CO}(2-1)$ & $0.26 \pm 0.05$ & $570.0 \pm 5.0$ & $15.0 \pm 5.0$ & $4.1 \pm 3.6$ \\
\hline & ${ }^{13} \mathrm{CO}(2-1)$ & $0.12 \pm 0.03$ & $573.2 \pm 1.0$ & $9.5 \pm 2.4$ & $1.2 \pm 0.4$ \\
\hline & ${ }^{13} \mathrm{CO}(1-0)$ & $<0.04$ & $\ldots$ & $\ldots$ & $\ldots$ \\
\hline & $\mathrm{C}^{18} \mathrm{O}(2-1)$ & $<0.01$ & $\cdots$ & $\cdots$ & $\cdots$ \\
\hline & $\mathrm{C}^{18} \mathrm{O}(1-0)$ & $<0.04$ & $\cdots$ & $\cdots$ & $\cdots$ \\
\hline & $\mathrm{HCO}^{+}(1-0)$ & $<0.06$ & $\cdots$ & $\cdots$ & $\cdots$ \\
\hline & $\mathrm{HCN}(1-0)$ & $<0.12$ & $\cdots$ & $\cdots$ & $\cdots$ \\
\hline & $\mathrm{CN}(1-0)$ & $<0.05$ & $\cdots$ & $\cdots$ & $\cdots$ \\
\hline & $\mathrm{HNC}(1-0)$ & $<0.06$ & $\cdots$ & $\cdots$ & $\cdots$ \\
\hline & $\mathrm{CCH}(1-0)$ & $<0.06$ & $\ldots$ & $\ldots$ & $\ldots$ \\
\hline \multirow[t]{10}{*}{ B-3 } & ${ }^{12} \mathrm{CO}(2-1)$ & $1.47 \pm 0.05$ & $711.7 \pm 1.2$ & $64.4 \pm 2.9$ & $100.6 \pm 5.9$ \\
\hline & ${ }^{13} \mathrm{CO}(2-1)$ & $0.07 \pm 0.01$ & $715.4 \pm 2.4$ & $68.1 \pm 5.6$ & $5.3 \pm 0.6$ \\
\hline & ${ }^{13} \mathrm{CO}(1-0)$ & $<0.04$ & $\ldots$ & $\ldots$ & $\ldots$ \\
\hline & $\mathrm{C}^{18} \mathrm{O}(2-1)$ & $<0.01$ & $\cdots$ & $\cdots$ & $\cdots$ \\
\hline & $\mathrm{C}^{18} \mathrm{O}(1-0)$ & $<0.04$ & $\ldots$ & $\ldots$ & $\ldots$ \\
\hline & $\mathrm{HCO}^{+}(1-0)$ & $0.40 \pm 0.02$ & $712.7 \pm 1.5$ & $65.5 \pm 3.5$ & $28.3 \pm 2.0$ \\
\hline & $\mathrm{HCN}(1-0)$ & $0.19 \pm 0.02$ & $701.9 \pm 2.8$ & $53.8 \pm 6.9$ & $11.7 \pm 1.8$ \\
\hline & $\mathrm{CN}(1-0)$ & $0.04 \pm 0.01$ & $697.6 \pm 6.5$ & $48.0 \pm 15.0$ & $2.2 \pm 0.9$ \\
\hline & $\mathrm{HNC}(1-0)$ & $0.11 \pm 0.02$ & $720.0 \pm 5.2$ & $63.0 \pm 12.0$ & $7.3 \pm 1.9$ \\
\hline & $\mathrm{CCH}(1-0)$ & $0.07 \pm 0.02$ & $726.9 \pm 5.7$ & $36.0 \pm 13.0$ & $2.7 \pm 1.3$ \\
\hline $\mathrm{C}-1$ & ${ }^{12} \mathrm{CO}(2-1)$ & $<0.12$ & $\ldots$ & $\ldots$ & $\ldots$ \\
\hline$(13: 25: 27.379)$ & ${ }^{13} \mathrm{CO}(2-1)$ & $0.03 \pm 0.02$ & $523.6 \pm 3.5$ & $10.8 \pm 8.2$ & $0.3 \pm 0.3$ \\
\hline$(-43.01 .05 .728)$ & ${ }^{13} \mathrm{CO}(1-0)$ & $<0.04$ & $\ldots$ & $\ldots$ & $\ldots$ \\
\hline
\end{tabular}


Table 2

(Continued)

\begin{tabular}{|c|c|c|c|c|c|}
\hline Line Identifier & Transition & $\begin{array}{l}\text { Peak } \\
(\mathrm{K})\end{array}$ & $\begin{array}{l}\text { Line Center } \\
\left(\mathrm{km} \mathrm{s}^{-1}\right)\end{array}$ & $\begin{array}{l}\text { FWHM } \\
\left(\mathrm{km} \mathrm{s}^{-1}\right)\end{array}$ & $\begin{array}{c}\text { Integral } \\
\left(\mathrm{K} \mathrm{km} \mathrm{s}^{-1}\right)\end{array}$ \\
\hline & $\mathrm{C}^{18} \mathrm{O}(2-1)$ & $<0.01$ & $\ldots$ & $\ldots$ & $\ldots$ \\
\hline & $\mathrm{C}^{18} \mathrm{O}(1-0)$ & $<0.04$ & $\ldots$ & $\ldots$ & $\ldots$ \\
\hline & $\mathrm{HCO}^{+}(1-0)$ & $<0.06$ & $\ldots$ & $\ldots$ & $\ldots$ \\
\hline & $\mathrm{HCN}(1-0)$ & $<0.06$ & $\ldots$ & $\ldots$ & $\ldots$ \\
\hline & $\mathrm{CN}(1-0)$ & $<0.04$ & $\ldots$ & $\ldots$ & $\ldots$ \\
\hline & $\mathrm{HNC}(1-0)$ & $<0.06$ & $\ldots$ & $\ldots$ & $\ldots$ \\
\hline & $\mathrm{CCH}(1-0)$ & $<0.06$ & $\ldots$ & $\ldots$ & $\ldots$ \\
\hline \multirow[t]{10}{*}{$\mathrm{C}-2$} & ${ }^{12} \mathrm{CO}(2-1)$ & $0.19 \pm 0.05$ & $560.0 \pm 5.0$ & $15.0 \pm 5.0$ & $3.1 \pm 3.6$ \\
\hline & ${ }^{13} \mathrm{CO}(2-1)$ & $0.18 \pm 0.05$ & $557.5 \pm 5.0$ & $5.0 \pm 3.0$ & $0.9 \pm 0.8$ \\
\hline & ${ }^{13} \mathrm{CO}(1-0)$ & $<0.04$ & $\ldots$ & $\ldots$ & $\ldots$ \\
\hline & $\mathrm{C}^{18} \mathrm{O}(2-1)$ & $<0.01$ & $\cdots$ & $\ldots$ & $\cdots$ \\
\hline & $\mathrm{C}^{18} \mathrm{O}(1-0)$ & $<0.04$ & $\ldots$ & $\ldots$ & $\ldots$ \\
\hline & $\mathrm{HCO}^{+}(1-0)$ & $<0.06$ & $\ldots$ & $\ldots$ & $\ldots$ \\
\hline & $\mathrm{HCN}(1-0)$ & $<0.06$ & $\ldots$ & $\ldots$ & $\ldots$ \\
\hline & $\mathrm{CN}(1-0)$ & $<0.04$ & $\ldots$ & $\ldots$ & $\ldots$ \\
\hline & $\mathrm{HNC}(1-0)$ & $<0.06$ & $\ldots$ & $\ldots$ & $\ldots$ \\
\hline & $\mathrm{CCH}(1-0)$ & $<0.06$ & $\ldots$ & $\ldots$ & $\ldots$ \\
\hline \multirow[t]{10}{*}{$\mathrm{C}-3$} & ${ }^{12} \mathrm{CO}(2-1)$ & $1.04 \pm 0.09$ & $649.1 \pm 1.9$ & $46.4 \pm 4.5$ & $51.2 \pm 6.6$ \\
\hline & ${ }^{13} \mathrm{CO}(2-1)$ & $0.04 \pm 0.01$ & $646.7 \pm 2.4$ & $46.4 \pm 5.6$ & $2.1 \pm 0.3$ \\
\hline & ${ }^{13} \mathrm{CO}(1-0)$ & $<0.04$ & $\ldots$ & $\ldots$ & $\ldots$ \\
\hline & $\mathrm{C}^{18} \mathrm{O}(2-1)$ & $<0.01$ & $\ldots$ & $\ldots$ & $\ldots$ \\
\hline & $\mathrm{C}^{18} \mathrm{O}(1-0)$ & $<0.04$ & $\ldots$ & $\ldots$ & $\ldots$ \\
\hline & $\mathrm{HCO}^{+}(1-0)$ & $0.64 \pm 0.02$ & $651.7 \pm 1.0$ & $56.3 \pm 2.2$ & $38.4 \pm 2.0$ \\
\hline & $\mathrm{HCN}(1-0)$ & $0.37 \pm 0.02$ & $648.4 \pm 1.4$ & $50.7 \pm 3.4$ & $20.0 \pm 1.8$ \\
\hline & $\mathrm{CN}(1-0)$ & $0.21 \pm 0.01$ & $649.2 \pm 1.4$ & $42.3 \pm 3.4$ & $9.6 \pm 1.0$ \\
\hline & $\mathrm{HNC}(1-0)$ & $0.19 \pm 0.02$ & $650.2 \pm 2.4$ & $38.0 \pm 5.8$ & $7.6 \pm 1.5$ \\
\hline & $\mathrm{CCH}(1-0)$ & $0.10 \pm 0.02$ & $664.8 \pm 5.7$ & $64.0 \pm 14.0$ & $6.6 \pm 1.8$ \\
\hline D-1 & ${ }^{12} \mathrm{CO}(2-1)$ & $0.86 \pm 0.05$ & $468.4 \pm 1.2$ & $42.0 \pm 2.9$ & $38.4 \pm 3.6$ \\
\hline$(13: 25: 27.918)$ & ${ }^{13} \mathrm{CO}(2-1)$ & $0.04 \pm 0.01$ & $459.1 \pm 1.8$ & $35.4 \pm 4.3$ & $1.4 \pm 0.2$ \\
\hline \multirow[t]{8}{*}{$(-43.01 .09 .025)$} & ${ }^{13} \mathrm{CO}(1-0)$ & $<0.03$ & $\ldots$ & $\ldots$ & $\ldots$ \\
\hline & $\mathrm{C}^{18} \mathrm{O}(2-1)$ & $<0.01$ & $\ldots$ & $\ldots$ & $\ldots$ \\
\hline & $\mathrm{C}^{18} \mathrm{O}(1-0)$ & $<0.04$ & $\ldots$ & $\ldots$ & $\ldots$ \\
\hline & $\mathrm{HCO}^{+}(1-0)$ & $0.83 \pm 0.02$ & $466.9 \pm 0.7$ & $46.3 \pm 1.6$ & $40.8 \pm 1.8$ \\
\hline & $\mathrm{HCN}(1-0)$ & $0.48 \pm 0.02$ & $465.2 \pm 1.2$ & $49.7 \pm 2.9$ & $25.5 \pm 2.0$ \\
\hline & $\mathrm{CN}(1-0)$ & $0.20 \pm 0.02$ & $465.9 \pm 1.7$ & $27.7 \pm 4.1$ & $5.8 \pm 1.2$ \\
\hline & $\mathrm{HNC}(1-0)$ & $0.16 \pm 0.02$ & $458.8 \pm 1.8$ & $39.6 \pm 4.3$ & $6.9 \pm 1.0$ \\
\hline & $\mathrm{CCH}(1-0)$ & $<0.07$ & $\ldots$ & $\ldots$ & $\ldots$ \\
\hline \multirow[t]{10}{*}{ D-2 } & ${ }^{12} \mathrm{CO}(2-1)$ & $0.36 \pm 0.05$ & $530.0 \pm 5.0$ & $20.0 \pm 5.0$ & $7.7 \pm 4.6$ \\
\hline & ${ }^{13} \mathrm{CO}(2-1)$ & $<0.01$ & $\ldots$ & $\ldots$ & $\ldots$ \\
\hline & ${ }^{13} \mathrm{CO}(1-0)$ & $<0.03$ & $\ldots$ & $\ldots$ & $\ldots$ \\
\hline & $\mathrm{C}^{18} \mathrm{O}(2-1)$ & $<0.01$ & $\ldots$ & $\ldots$ & $\ldots$ \\
\hline & $\mathrm{C}^{18} \mathrm{O}(1-0)$ & $<0.04$ & $\cdots$ & $\ldots$ & $\cdots$ \\
\hline & $\mathrm{HCO}^{+}(1-0)$ & $<0.07$ & $\ldots$ & $\ldots$ & $\ldots$ \\
\hline & $\mathrm{HCN}(1-0)$ & $<0.06$ & $\ldots$ & $\ldots$ & $\ldots$ \\
\hline & $\mathrm{CN}(1-0)$ & $<0.06$ & $\ldots$ & $\ldots$ & $\ldots$ \\
\hline & $\mathrm{HNC}(1-0)$ & $<0.06$ & $\cdots$ & $\cdots$ & $\cdots$ \\
\hline & $\mathrm{CCH}(1-0)$ & $<0.07$ & $\ldots$ & $\ldots$ & $\ldots$ \\
\hline \multirow[t]{10}{*}{ D-3 } & ${ }^{12} \mathrm{CO}(2-1)$ & $<0.23$ & $\ldots$ & $\ldots$ & $\ldots$ \\
\hline & ${ }^{13} \mathrm{CO}(2-1)$ & $0.02 \pm 0.01$ & $609.9 \pm 3.4$ & $17.0 \pm 8.1$ & $0.3 \pm 0.2$ \\
\hline & ${ }^{13} \mathrm{CO}(1-0)$ & $<0.03$ & $\ldots$ & $\ldots$ & $\ldots$ \\
\hline & $\mathrm{C}^{18} \mathrm{O}(2-1)$ & $<0.01$ & $\ldots$ & $\ldots$ & $\ldots$ \\
\hline & $\mathrm{C}^{18} \mathrm{O}(1-0)$ & $<0.04$ & $\ldots$ & $\ldots$ & $\ldots$ \\
\hline & $\mathrm{HCO}^{+}(1-0)$ & $<0.07$ & $\ldots$ & $\ldots$ & $\ldots$ \\
\hline & $\mathrm{HCN}(1-0)$ & $<0.06$ & $\ldots$ & $\ldots$ & $\ldots$ \\
\hline & $\mathrm{CN}(1-0)$ & $<0.06$ & $\ldots$ & $\ldots$ & $\ldots$ \\
\hline & $\mathrm{HNC}(1-0)$ & $<0.06$ & $\ldots$ & $\ldots$ & $\ldots$ \\
\hline & $\mathrm{CCH}(1-0)$ & $<0.07$ & $\ldots$ & $\ldots$ & $\ldots$ \\
\hline \multirow[t]{6}{*}{ D-4 } & ${ }^{12} \mathrm{CO}(2-1)$ & $0.13 \pm 0.05$ & $704.8 \pm 7.9$ & $44.0 \pm 18.0$ & $6.1 \pm 3.3$ \\
\hline & ${ }^{13} \mathrm{CO}(2-1)$ & $<0.01$ & $\ldots$ & $\ldots$ & $\ldots$ \\
\hline & ${ }^{13} \mathrm{CO}(1-0)$ & $<0.03$ & $\ldots$ & $\ldots$ & $\ldots$ \\
\hline & $\mathrm{C}^{18} \mathrm{O}(2-1)$ & $<0.01$ & $\ldots$ & $\ldots$ & $\ldots$ \\
\hline & $\mathrm{C}^{18} \mathrm{O}(1-0)$ & $<0.04$ & $\ldots$ & $\ldots$ & $\ldots$ \\
\hline & $\mathrm{HCO}^{+}(1-0)$ & $<0.07$ & $\ldots$ & $\ldots$ & $\ldots$ \\
\hline
\end{tabular}


Table 2

(Continued)

\begin{tabular}{|c|c|c|c|c|c|}
\hline Line Identifier & Transition & $\begin{array}{l}\text { Peak } \\
(\mathrm{K})\end{array}$ & $\begin{array}{l}\text { Line Center } \\
\left(\mathrm{km} \mathrm{s}^{-1}\right)\end{array}$ & $\begin{array}{l}\text { FWHM } \\
\left(\mathrm{km} \mathrm{s}^{-1}\right)\end{array}$ & $\begin{array}{c}\text { Integral } \\
\left(\mathrm{K} \mathrm{km} \mathrm{s}^{-1}\right)\end{array}$ \\
\hline & $\mathrm{HCN}(1-0)$ & $<0.06$ & $\cdots$ & $\cdots$ & $\cdots$ \\
\hline & $\mathrm{CN}(1-0)$ & $<0.06$ & $\ldots$ & $\ldots$ & $\ldots$ \\
\hline & $\mathrm{HNC}(1-0)$ & $<0.06$ & $\ldots$ & $\ldots$ & $\ldots$ \\
\hline & $\mathrm{CCH}(1-0)$ & $<0.07$ & $\ldots$ & $\ldots$ & $\ldots$ \\
\hline E-1 & ${ }^{12} \mathrm{CO}(2-1)$ & $1.05 \pm 0.03$ & $374.5 \pm 1.0$ & $61.2 \pm 2.3$ & $68.3 \pm 3.3$ \\
\hline$(13: 25: 27.918)$ & ${ }^{13} \mathrm{CO}(2-1)$ & $0.07 \pm 0.01$ & $376.1 \pm 2.0$ & $49.3 \pm 4.7$ & $3.5 \pm 0.4$ \\
\hline \multirow[t]{8}{*}{$(-43.01 .15 .132)$} & ${ }^{13} \mathrm{CO}(1-0)$ & $<0.04$ & $\ldots$ & $\ldots$ & $\ldots$ \\
\hline & $\mathrm{C}^{18} \mathrm{O}(2-1)$ & $<0.01$ & $\ldots$ & $\ldots$ & $\ldots$ \\
\hline & $\mathrm{C}^{18} \mathrm{O}(1-0)$ & $<0.04$ & $\ldots$ & $\ldots$ & $\ldots$ \\
\hline & $\mathrm{HCO}^{+}(1-0)$ & $0.42 \pm 0.02$ & $374.2 \pm 1.4$ & $52.8 \pm 3.4$ & $23.6 \pm 2.0$ \\
\hline & $\mathrm{HCN}(1-0)$ & $0.19 \pm 0.02$ & $372.8 \pm 1.2$ & $46.2 \pm 6.3$ & $9.5 \pm 1.7$ \\
\hline & $\mathrm{CN}(1-0)$ & $0.09 \pm 0.02$ & $371.2 \pm 1.8$ & $19.3 \pm 4.4$ & $1.8 \pm 0.5$ \\
\hline & $\mathrm{HNC}(1-0)$ & $0.05 \pm 0.01$ & $359.0 \pm 11.0$ & $105.0 \pm 31.0$ & $6.2 \pm 2.2$ \\
\hline & $\mathrm{CCH}(1-0)$ & $0.07 \pm 0.02$ & $399.1 \pm 5.3$ & $44.0 \pm 15.0$ & $3.3 \pm 1.4$ \\
\hline \multirow[t]{10}{*}{ E-2 } & ${ }^{12} \mathrm{CO}(2-1)$ & $<0.23$ & $\ldots$ & $\ldots$ & $\ldots$ \\
\hline & ${ }^{13} \mathrm{CO}(2-1)$ & $0.11 \pm 0.04$ & $543.6 \pm 1.6$ & $9.1 \pm 3.8$ & $1.0 \pm 0.6$ \\
\hline & ${ }^{13} \mathrm{CO}(1-0)$ & $<0.04$ & $\ldots$ & ... & $\ldots$ \\
\hline & $\mathrm{C}^{18} \mathrm{O}(2-1)$ & $<0.01$ & $\ldots$ & $\ldots$ & $\ldots$ \\
\hline & $\mathrm{C}^{18} \mathrm{O}(1-0)$ & $<0.04$ & $\ldots$ & $\ldots$ & $\ldots$ \\
\hline & $\mathrm{HCO}^{+}(1-0)$ & $0.09 \pm 0.03$ & $544.6 \pm 2.1$ & $13.6 \pm 4.9$ & $1.3 \pm 0.6$ \\
\hline & $\mathrm{HCN}(1-0)$ & $<0.06$ & $\ldots$ & $\ldots$ & $\ldots$ \\
\hline & $\mathrm{CN}(1-0)$ & $<0.04$ & $\ldots$ & $\ldots$ & $\ldots$ \\
\hline & $\mathrm{HNC}(1-0)$ & $<0.06$ & $\cdots$ & $\cdots$ & $\cdots$ \\
\hline & $\mathrm{CCH}(1-0)$ & $<0.07$ & $\ldots$ & $\ldots$ & $\ldots$ \\
\hline \multirow[t]{10}{*}{ E-3 } & ${ }^{12} \mathrm{CO}(2-1)$ & $0.37 \pm 0.07$ & $590.0 \pm 3.7$ & $39.8 \pm 8.7$ & $15.9 \pm 4.6$ \\
\hline & ${ }^{13} \mathrm{CO}(2-1)$ & $0.03 \pm 0.01$ & $584.0 \pm 1.2$ & $8.2 \pm 2.9$ & $0.3 \pm 0.1$ \\
\hline & ${ }^{13} \mathrm{CO}(1-0)$ & $<0.04$ & $\ldots$ & $\ldots$ & $\ldots$ \\
\hline & $\mathrm{C}^{18} \mathrm{O}(2-1)$ & $<0.01$ & $\ldots$ & $\ldots$ & $\ldots$ \\
\hline & $\mathrm{C}^{18} \mathrm{O}(1-0)$ & $<0.04$ & $\ldots$ & $\ldots$ & $\ldots$ \\
\hline & $\mathrm{HCO}^{+}(1-0)$ & $<0.06$ & $\cdots$ & $\cdots$ & $\cdots$ \\
\hline & $\mathrm{HCN}(1-0)$ & $<0.06$ & $\ldots$ & $\ldots$ & $\ldots$ \\
\hline & $\mathrm{CN}(1-0)$ & $<0.04$ & $\cdots$ & $\cdots$ & $\ldots$ \\
\hline & $\mathrm{HNC}(1-0)$ & $<0.06$ & $\ldots$ & $\ldots$ & $\ldots$ \\
\hline & $\mathrm{CCH}(1-0)$ & $<0.07$ & $\ldots$ & $\ldots$ & $\ldots$ \\
\hline $\mathrm{F}-1$ & ${ }^{12} \mathrm{CO}(2-1)$ & $<0.15$ & $\ldots$ & $\ldots$ & $\ldots$ \\
\hline$(13: 25: 26.819)$ & ${ }^{13} \mathrm{CO}(2-1)$ & $0.19 \pm 0.02$ & $545.6 \pm 0.3$ & $5.3 \pm 0.9$ & $1.1 \pm 0.2$ \\
\hline \multirow[t]{8}{*}{$(-43.01 .13 .585)$} & ${ }^{13} \mathrm{CO}(1-0)$ & $0.16 \pm 0.03$ & $549.2 \pm 0.3$ & $2.3 \pm 0.5$ & $0.4 \pm 0.1$ \\
\hline & $\mathrm{C}^{18} \mathrm{O}(2-1)$ & $<0.01$ & $\ldots$ & $\ldots$ & .. \\
\hline & $\mathrm{C}^{18} \mathrm{O}(1-0)$ & $<0.04$ & $\ldots$ & $\ldots$ & $\ldots$ \\
\hline & $\mathrm{HCO}^{+}(1-0)$ & $<0.06$ & $\ldots$ & $\ldots$ & $\ldots$ \\
\hline & $\mathrm{HCN}(1-0)$ & $<0.06$ & $\cdots$ & $\cdots$ & $\cdots$ \\
\hline & $\mathrm{CN}(1-0)$ & $<0.04$ & $\ldots$ & $\ldots$ & $\ldots$ \\
\hline & $\mathrm{HNC}(1-0)$ & $<0.06$ & $\ldots$ & $\ldots$ & $\ldots$ \\
\hline & $\mathrm{CCH}(1-0)$ & $<0.07$ & $\ldots$ & $\ldots$ & $\ldots$ \\
\hline \multirow[t]{10}{*}{$\mathrm{F}-2$} & ${ }^{12} \mathrm{CO}(2-1)$ & $1.79 \pm 0.11$ & $581.0 \pm 0.6$ & $18.4 \pm 1.3$ & $35.1 \pm 3.3$ \\
\hline & ${ }^{13} \mathrm{CO}(2-1)$ & $0.51 \pm 0.03$ & $581.2 \pm 0.4$ & $16.6 \pm 1.0$ & $8.9 \pm 0.7$ \\
\hline & ${ }^{13} \mathrm{CO}(1-0)$ & $0.47 \pm 0.03$ & $584.9 \pm 0.4$ & $11.5 \pm 0.9$ & $5.7 \pm 0.6$ \\
\hline & $\mathrm{C}^{18} \mathrm{O}(2-1)$ & $0.06 \pm 0.01$ & $582.4 \pm 1.4$ & $13.3 \pm 3.3$ & $0.8 \pm 0.3$ \\
\hline & $\mathrm{C}^{18} \mathrm{O}(1-0)$ & $<0.04$ & $\ldots$ & $\ldots$ & $\ldots$ \\
\hline & $\mathrm{HCO}^{+}(1-0)$ & $<0.06$ & $\ldots$ & $\ldots$ & $\ldots$ \\
\hline & $\mathrm{HCN}(1-0)$ & $<0.06$ & $\ldots$ & $\ldots$ & $\ldots$ \\
\hline & $\mathrm{CN}(1-0)$ & $<0.04$ & $\ldots$ & $\ldots$ & $\ldots$ \\
\hline & $\mathrm{HNC}(1-0)$ & $<0.06$ & $\cdots$ & $\cdots$ & $\cdots$ \\
\hline & $\mathrm{CCH}(1-0)$ & $<0.07$ & $\ldots$ & $\ldots$ & $\ldots$ \\
\hline G-1 & ${ }^{12} \mathrm{CO}(2-1)$ & $0.25 \pm 0.12$ & $442.4 \pm 5.4$ & $23.0 \pm 13.0$ & $6.1 \pm 4.6$ \\
\hline$(13: 25: 28.556)$ & ${ }^{13} \mathrm{CO}(2-1)$ & $0.05 \pm 0.01$ & $469.3 \pm 8.2$ & $46.0 \pm 20.0$ & $2.2 \pm 1.1$ \\
\hline \multirow{7}{*}{$(-43.01 .21 .565)$} & ${ }^{13} \mathrm{CO}(1-0)$ & $<0.04$ & $\ldots$ & $\ldots$ & $\ldots$ \\
\hline & $\mathrm{C}^{18} \mathrm{O}(2-1)$ & $<0.01$ & $\ldots$ & $\ldots$ & $\ldots$ \\
\hline & $\mathrm{C}^{18} \mathrm{O}(1-0)$ & $<0.04$ & $\ldots$ & $\ldots$ & $\ldots$ \\
\hline & $\mathrm{HCO}^{+}(1-0)$ & $<0.06$ & $\ldots$ & $\ldots$ & $\ldots$ \\
\hline & $\mathrm{HCN}(1-0)$ & $<0.06$ & $\ldots$ & $\ldots$ & $\ldots$ \\
\hline & $\mathrm{CN}(1-0)$ & $<0.04$ & $\ldots$ & $\ldots$ & $\ldots$ \\
\hline & $\mathrm{HNC}(1-0)$ & $<0.06$ & $\ldots$ & $\ldots$ & $\ldots$ \\
\hline
\end{tabular}


Table 2

(Continued)

\begin{tabular}{|c|c|c|c|c|c|}
\hline Line Identifier & Transition & $\begin{array}{l}\text { Peak } \\
(\mathrm{K})\end{array}$ & $\begin{array}{l}\text { Line Center } \\
\left(\mathrm{km} \mathrm{s}^{-1}\right)\end{array}$ & $\begin{array}{c}\text { FWHM } \\
\left(\mathrm{km} \mathrm{s}^{-1}\right)\end{array}$ & $\begin{array}{c}\text { Integral } \\
\left(\mathrm{K} \mathrm{km} \mathrm{s}^{-1}\right)\end{array}$ \\
\hline & $\mathrm{CCH}(1-0)$ & $<0.07$ & $\ldots$ & $\ldots$ & $\ldots$ \\
\hline \multirow[t]{10}{*}{ G-2 } & ${ }^{12} \mathrm{CO}(2-1)$ & $2.16 \pm 0.12$ & $507.6 \pm 0.6$ & $23.6 \pm 1.5$ & $54.5 \pm 4.6$ \\
\hline & ${ }^{13} \mathrm{CO}(2-1)$ & $1.22 \pm 0.04$ & $509.8 \pm 0.3$ & $18.9 \pm 0.7$ & $24.6 \pm 1.3$ \\
\hline & ${ }^{13} \mathrm{CO}(1-0)$ & $0.52 \pm 0.04$ & $511.0 \pm 0.6$ & $18.2 \pm 1.4$ & $10.1 \pm 1.0$ \\
\hline & $\mathrm{C}^{18} \mathrm{O}(2-1)$ & $0.09 \pm 0.02$ & $512.5 \pm 1.8$ & $19.2 \pm 4.3$ & $1.9 \pm 0.6$ \\
\hline & $\mathrm{C}^{18} \mathrm{O}(1-0)$ & $<0.04$ & $\ldots$ & $\ldots$ & $\ldots$ \\
\hline & $\mathrm{HCO}^{+}(1-0)$ & $0.28 \pm 0.05$ & $503.9 \pm 0.9$ & $10.4 \pm 2.1$ & $3.1 \pm 0.8$ \\
\hline & $\mathrm{HCN}(1-0)$ & $0.14 \pm 0.03$ & $510.7 \pm 1.7$ & $17.6 \pm 4.1$ & $2.6 \pm 0.8$ \\
\hline & $\mathrm{CN}(1-0)$ & $<0.04$ & $\ldots$ & $\ldots$ & $\ldots$ \\
\hline & $\mathrm{HNC}(1-0)$ & $<0.06$ & $\ldots$ & $\ldots$ & $\ldots$ \\
\hline & $\mathrm{CCH}(1-0)$ & $<0.07$ & $\ldots$ & $\ldots$ & $\ldots$ \\
\hline $\mathrm{H}-1$ & ${ }^{12} \mathrm{CO}(2-1)$ & $2.00 \pm 0.10$ & $524.2 \pm 0.6$ & $22.3 \pm 1.3$ & $47.6 \pm 3.6$ \\
\hline$(13: 25: 28.556)$ & ${ }^{13} \mathrm{CO}(2-1)$ & $1.23 \pm 0.09$ & $521.7 \pm 0.6$ & $14.9 \pm 1.4$ & $19.6 \pm 2.3$ \\
\hline \multirow[t]{8}{*}{$(-43.01 .20 .343)$} & ${ }^{13} \mathrm{CO}(1-0)$ & $1.02 \pm 0.05$ & $523.8 \pm 0.3$ & $11.3 \pm 0.6$ & $12.2 \pm 0.9$ \\
\hline & $\mathrm{C}^{18} \mathrm{O}(2-1)$ & $0.35 \pm 0.03$ & $518.4 \pm 0.3$ & $6.3 \pm 0.5$ & $2.3 \pm 0.3$ \\
\hline & $\mathrm{C}^{18} \mathrm{O}(1-0)$ & $<0.04$ & $\ldots$ & $\ldots$ & $\ldots$ \\
\hline & $\mathrm{HCO}^{+}(1-0)$ & $0.17 \pm 0.03$ & $520.7 \pm 1.4$ & $15.3 \pm 3.3$ & $2.8 \pm 0.8$ \\
\hline & $\mathrm{HCN}(1-0)$ & $0.12 \pm 0.04$ & $521.1 \pm 2.7$ & $18.4 \pm 6.5$ & $2.4 \pm 1.1$ \\
\hline & $\mathrm{CN}(1-0)$ & $<0.04$ & $\ldots$ & $\ldots$ & $\ldots$ \\
\hline & $\mathrm{HNC}(1-0)$ & $<0.06$ & $\ldots$ & $\ldots$ & $\ldots$ \\
\hline & $\mathrm{CCH}(1-0)$ & $<0.07$ & $\ldots$ & $\ldots$ & $\cdots$ \\
\hline \multirow[t]{10}{*}{$\mathrm{H}-2$} & ${ }^{12} \mathrm{CO}(2-1)$ & $<0.08$ & $\ldots$ & $\ldots$ & $\ldots$ \\
\hline & ${ }^{13} \mathrm{CO}(2-1)$ & $<0.01$ & $\ldots$ & $\ldots$ & $\ldots$ \\
\hline & ${ }^{13} \mathrm{CO}(1-0)$ & $0.09 \pm 0.03$ & $559.9 \pm 0.9$ & $6.8 \pm 2.1$ & $0.7 \pm 0.3$ \\
\hline & $\mathrm{C}^{18} \mathrm{O}(2-1)$ & $0.04 \pm 0.01$ & $558.1 \pm 1.0$ & $9.9 \pm 2.4$ & $0.4 \pm 0.1$ \\
\hline & $\mathrm{C}^{18} \mathrm{O}(1-0)$ & $<0.04$ & $\ldots$ & $\ldots$ & $\ldots$ \\
\hline & $\mathrm{HCO}^{+}(1-0)$ & $<0.07$ & $\ldots$ & $\ldots$ & $\ldots$ \\
\hline & $\mathrm{HCN}(1-0)$ & $<0.06$ & $\ldots$ & $\ldots$ & $\ldots$ \\
\hline & $\mathrm{CN}(1-0)$ & $<0.04$ & $\ldots$ & $\ldots$ & $\ldots$ \\
\hline & $\mathrm{HNC}(1-0)$ & $<0.06$ & $\ldots$ & $\ldots$ & $\ldots$ \\
\hline & $\mathrm{CCH}(1-0)$ & $<0.07$ & $\ldots$ & $\ldots$ & $\ldots$ \\
\hline
\end{tabular}

Note. For non-detected features, a $1 \sigma$ upper limit is given. Spectral features attributed to the CND are in bold.

(This table is available in machine-readable form.)

${ }^{12} \mathrm{CO}$ for features B-1 and G-2 are around 2 and 12, respectively.

Using the ${ }^{13} \mathrm{CO}(2-1)$ to ${ }^{13} \mathrm{CO}(1-0)$ ratio, we can constrain the excitation temperature of the gas (Aalto et al. 1995; Meier \& Turner 2000). Spectral features A-1, B-1, F-2, and $\mathrm{H}-1$ have ${ }^{13} \mathrm{CO}$ excitation temperatures over $10 \mathrm{~K}$, while the feature $\mathrm{G}-2$ has an excitation temperature of $\sim 20 \mathrm{~K}$ in the optically thin limit. For the $\mathrm{CND}$, the limits from the ${ }^{13} \mathrm{CO}(2-1)$ to ${ }^{13} \mathrm{CO}(1-0)$ ratio give lower limits on the excitation temperature of the gas of about $15 \mathrm{~K}$ for the outer regions of the $\mathrm{CND}$ and $10 \mathrm{~K}$ for the inner regions assuming optically thin gas.

\subsubsection{Dense Gas in the CND}

$\mathrm{HCN}, \mathrm{HCO}^{+}, \mathrm{HNC}, \mathrm{CN}$, and $\mathrm{CCH}$ are molecules that have high $\mathrm{H}_{2}$ critical densities $n_{\text {crit }}{ }^{16}$ The critical densities of these molecules for their respective transitions are $\mathrm{CCH}: 1.4 \times$ $10^{5} \mathrm{~cm}^{-3}, \mathrm{HCO}^{+}: 1.9 \times 10^{5} \mathrm{~cm}^{-3}, \mathrm{HCN}: 8.7 \times 10^{5} \mathrm{~cm}^{-3}$, $\mathrm{CN}: 1.3 \times 10^{6} \mathrm{~cm}^{-3}$, and HNC: $2.9 \times 10^{6} \mathrm{~cm}^{-3}$ (Green \&

\footnotetext{
16 The critical density here is listed as the density at which the spontaneous emission coefficient $\left(A_{i j}\right.$, between levels $\mathrm{i}$ and $\left.\mathrm{j}\right)$ is equal to the collision rate (where ${ }_{A_{i j}}$ we adopt a collision coefficient $C_{i j}$ for gas at $10 \mathrm{~K}$ ), $\mathrm{n}_{\text {crit }} \simeq \frac{A_{i j}}{C_{i j}(10 \mathrm{~K})}$. Radiative trapping and collisions with electrons may reduce the effective critical density of these transitions significantly below the quoted values (Shirley 2015; Goldsmith \& Kauffmann 2017).
}

Thaddeus 1974; Flower 1999; Lique et al. 2010). Unlike the low- $J$ transitions of $\mathrm{CO}$, the $J=1-0$ transition of these molecules are excited at gas densities $\gtrsim 10^{4} \mathrm{~cm}^{-3}$. They dominate emission from the CND but not the molecular arms. So the average mass-weighted gas density is higher in the CND than in the molecular arms.

More subtle distinctions between the dense gas tracers can provide insights into the physical and chemical conditions of the CND. Ratios between $\mathrm{HCN}, \mathrm{HCO}^{+}$, and $\mathrm{HNC}$ provide constraints on the gas density and sources of gas chemistry/ excitation. The $\mathrm{HCN} / \mathrm{HCO}^{+}, \mathrm{HCN} / \mathrm{HNC}$, and $\mathrm{HNC} / \mathrm{HCO}^{+}$ $J=1-0$ line ratios tend to be of order unity, with possible deviations up to an order of magnitude (e.g., Baan et al. 2008; Krips et al. 2008). Various models of photon-dominated regions (PDRs), X-ray dominated regions (XDRs), cosmic ray dissociation regions, and mechanical heating/high temperature chemistry have been applied to explain the observed ratios and their variations (e.g., Meijerink et al. 2006, 2007; Loenen et al. 2008; Harada et al. 2010; Meijerink et al. 2011). CN and $\mathrm{CCH}$ are generally thought to trace molecular gas in a partially ionized (mainly $\mathrm{C}^{+}$) state associated with PDRs and XDRs (Wootten et al. 1980; Sternberg \& Dalgarno 1995; RodriguezFranco et al. 1998; Meier \& Turner 2005; Boger \& Sternberg 2006; Meijerink et al. 2007). 
Table 3

Ratios of Selected Molecular Transitions

\begin{tabular}{|c|c|c|c|c|c|c|c|c|c|c|}
\hline ID & $\begin{array}{l}\frac{{ }^{12} \mathrm{CO}(2-1)}{{ }^{13} \mathrm{CO}(2-1)} \\
\end{array}$ & 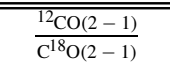 & $\begin{array}{l}\frac{{ }^{13} \mathrm{CO}(2-1)}{{ }^{13} \mathrm{CO}(1-0)} \\
\end{array}$ & $\frac{\overline{C^{18} \mathrm{O}(2-1)}}{\mathrm{C}^{18} \mathrm{O}(1-0)}$ & $\frac{\overline{\overline{1}^{13} \mathrm{CO}(2-1)}}{\mathrm{C}^{18} \mathrm{O}(2-1)}$ & 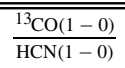 & $\frac{\mathrm{HCN}(1-0)}{\mathrm{HCO}^{+}(1-0)}$ & $\begin{array}{l}\operatorname{HCN}(1-0) \\
\operatorname{HNC}(1-0)\end{array}$ & $\frac{\mathrm{HCN}(1-0)}{\mathrm{CN}(1-0)}$ & $\overline{\frac{\mathrm{HCN}(1-0)}{\mathrm{CCH}(1-0)}}$ \\
\hline A-2 & $1.2 \pm 0.4$ & $>14.8$ & $1.5 \pm 0.4$ & $\ldots$ & $>12.8$ & $>3.1$ & $\ldots$ & $\ldots$ & $\cdots$ & $\ldots$ \\
\hline B-1 & $5.1 \pm 0.8$ & $111.9 \pm 61.8$ & $1.5 \pm 0.3$ & $>0.5$ & $21.8 \pm 12.1$ & $3.5 \pm 1.4$ & $0.6 \pm 0.3$ & $>1.4$ & $>1.8$ & $>1.3$ \\
\hline B-2 & $2.2 \pm 0.7$ & $>25.1$ & $>3.1$ & $\ldots$ & $>11.7$ & $\ldots$ & $\ldots$ & $\ldots$ & $\ldots$ & $\ldots$ \\
\hline B-3 & $20.0 \pm 3.3$ & $>144.1$ & $>1.9$ & $\cdots$ & $>7.2$ & $<0.2$ & $0.5 \pm 0.1$ & $1.7 \pm 0.4$ & $4.3 \pm 1.3$ & $2.6 \pm 0.9$ \\
\hline C-1 & $<4.5$ & $\ldots$ & $>0.7$ & $\cdots$ & $>2.9$ & $\ldots$ & $\ldots$ & $\ldots$ & $\ldots$ & $\ldots$ \\
\hline D-1 & $23.5 \pm 4.4$ & $>99.4$ & $>1.1$ & $\ldots$ & $>4.2$ & $<0.1$ & $0.6 \pm 0.1$ & $3.0 \pm 0.4$ & $2.4 \pm 0.4$ & $>7.0$ \\
\hline D-2 & $>42.9$ & $>41.4$ & $\ldots$ & $\ldots$ & $\ldots$ & $\cdots$ & $\ldots$ & $\ldots$ & $\ldots$ & $\ldots$ \\
\hline D-3 & $<13.1$ & $\ldots$ & $>0.5$ & $\ldots$ & $>2.0$ & $\cdots$ & $\cdots$ & $\cdots$ & $\cdots$ & $\cdots$ \\
\hline D-4 & $>15.9$ & $>15.4$ & $\cdots$ & $\cdots$ & $\cdots$ & $\cdots$ & $\ldots$ & $\ldots$ & $\ldots$ & $\ldots$ \\
\hline E-1 & $16.0 \pm 2.6$ & $>101.7$ & $>1.9$ & $\cdots$ & $>6.4$ & $<0.2$ & $0.5 \pm 0.1$ & $3.5 \pm 0.9$ & $2.2 \pm 0.5$ & $2.7 \pm 0.7$ \\
\hline E-2 & $<2.2$ & $\ldots$ & $>3.0$ & $\cdots$ & $>10.3$ & $\cdots$ & $<0.7$ & $\ldots$ & $\ldots$ & $\ldots$ \\
\hline G-2 & $1.8 \pm 0.3$ & $22.9 \pm 5.7$ & $2.4 \pm 0.3$ & $>2.6$ & $12.9 \pm 3.2$ & $3.7 \pm 0.8$ & $0.5 \pm 0.1$ & $>2.4$ & $>3.2$ & $>2.1$ \\
\hline H-1 & $1.6 \pm 0.3$ & $5.7 \pm 1.0$ & $1.2 \pm 0.2$ & $>9.8$ & $3.5 \pm 0.6$ & $8.4 \pm 2.7$ & $0.7 \pm 0.3$ & $>1.9$ & $>3.1$ & $>1.8$ \\
\hline $\mathrm{H}-2$ & $\ldots$ & $<2.1$ & $<0.1$ & $>1.1$ & $<0.3$ & $>1.5$ & $\ldots$ & $\ldots$ & $\ldots$ & $\ldots$ \\
\hline
\end{tabular}

Note. $T_{\mathrm{pk}}$ ratios of the emission features. Limits are given for values where only a single transition was detected. Spectral features attributed to the CND are in bold.

The behavior of these dense gas line ratios can be subtle and complex, depending on the nature and form of the energetics controlling gas excitation/ionization, but some summarizing statements are possible. XDRs tend to enhance $\mathrm{HCO}^{+}$ modestly and $\mathrm{CN}$ strongly relative to $\mathrm{HCN}$ and $\mathrm{HNC}$, compared to PDRs, producing $\mathrm{HCN} / \mathrm{HCO}^{+}$ratios below unity. However, PDRs with densities of $\sim 10^{4} \mathrm{~cm}^{-3}$ and high columns can also produce $\mathrm{HCN} / \mathrm{HCO}^{+}<1$ (e.g., Meijerink et al. 2007). New calculations of photodissociation rates for HNC find values larger than HCN (Aguado et al. 2017), indicating PDRs probably favor $\mathrm{HCN} / \mathrm{HNC}>1$, consistent with what is seen in the nearby starburst IC 342 (Meier \& Turner 2005). Increasing cosmic ray ionization generally favors increasing abundances of $\mathrm{HCO}^{+}$, relative to $\mathrm{HCN}$, at high densities ( $n \sim 10^{5} \mathrm{~cm}^{-3}$; e.g., Meijerink et al. 2011). The addition of mechanical heating or high temperature chemistry tends to elevate $\mathrm{HCN}$ and reduce $\mathrm{HNC}$ and $\mathrm{HCO}^{+}$abundances (e.g., Schilke et al. 1992; Loenen et al. 2008; Harada et al. 2010; Izumi et al. 2013).

Comparing the observed peak values of the dense gas tracers to theoretical work, excitation sources and conditions can potentially be constrained. Throughout the map, we find that $\mathrm{HCO}^{+}(1-0)$ is brighter than $\mathrm{HCN}(1-0)$, with an $\mathrm{HCN}(1-0) /$ $\mathrm{HCO}^{+}(1-0)$ ratio near 0.6 everywhere they are both detected. The observed $\mathrm{HCN}(1-0) / \mathrm{HCO}^{+}(1-0)$ ratio is on the low end compared to what is found for Seyfert galaxies such as NGC 1097 and NGC 1068 (Kohno 2003). Espada et al. (2017) find $\mathrm{HCN}(4-3) / \mathrm{HCO}+(4-3)$ integrated intensity emission ratio is also very low $(<0.5)$ in molecular clouds a few tens of pc away from the center in the nuclear filaments, unlike other low luminosity AGNs. We can place Cen A on Figure 1 of Kohno (2003) by adopting an $\mathrm{I}\left[{ }^{12} \mathrm{CO}(2-1)\right] / \mathrm{I}\left[{ }^{12} \mathrm{CO}(1-0)\right]$ ratio of 0.9 . Using our observed $\mathrm{HCN}(1-0) / \mathrm{HCO}^{+}(1-0)$ ratio and the ${ }^{12} \mathrm{CO}(2-1)$ from the science verification data, Cen A would be located among the galaxies labeled as "Composite" AGNs (Kohno 2003).

The $\mathrm{HCN}(1-0) / \mathrm{HNC}(1-0)$ intensity ratio is typically near unity in molecular environments (e.g., Aalto et al. 2002; Baan et al. 2008). I[HCN(1-0)]/I[HNC(1-0)] ratios toward the CND of Cen A are between 1.5-3.5, strongly favoring $\mathrm{HCN}$ over HNC and consistent with densities of $\sim 10^{4} \mathrm{~cm}^{-3}$ and strong radiation fields/high temperatures. The combination of the $\mathrm{HCN} / \mathrm{HCO}^{+}, \mathrm{HCN} / \mathrm{HNC}$, and $\mathrm{HCO}^{+} / \mathrm{HNC}$ ratios also place Cen A in the PDR parameter space of Baan et al. (2008), but $\mathrm{HCO}^{+}$remains near the top (XDR) envelope of the PDR range. This is similar to results by Kohno (2003), including ratios with HNC favoring PDRs, but with some "composite" (AGN and starburst) nature.

In the evolved pure starburst $\mathrm{M} 82, \mathrm{HCO}^{+}$is indeed brighter than $\mathrm{HCN}$ (Nguyen et al. 1992), so elevated $\mathrm{HCO}^{+} / \mathrm{HCN}$ ratios may not exclusively reflect XDR conditions. "Composite" AGNs containing a nuclear starburst (SB) may enhance $\mathrm{HCO}^{+}$emission through supernova explosions, elevating the intense cosmic ionization flux (e.g., Nguyen et al. 1992; Meijerink et al. 2011). Marconi et al. (2000) detected Pa $\alpha$ in the nuclear region of Cen $\mathrm{A}$ and suggests the star formation in the circumnuclear region to be $\sim 0.3 M_{\odot} \mathrm{yr}^{-1}$.

Recent results from NGC 1068 (Garcia-Burillo et al. 2014) and NGC 1097 (Martín et al. 2015) suggest that the differentiation of AGN/SB might be attributable to gas density or mechanical heating (Loenen et al. 2008; Izumi et al. 2013, 2016) instead of AGN heating. In fact, adding some mechanical heating to PDRs models (Loenen et al. 2008) does a better job of matching Cen A's $\mathrm{HCN} / \mathrm{HCO}^{+}, \mathrm{HCN} /$ $\mathrm{HNC}$, and $\mathrm{HNC} / \mathrm{HCO}^{+}$ratios.

The $\mathrm{HCN}(1-0) / \mathrm{CN}(1-0)$ ratio is also a useful diagnostic. Typically $\mathrm{CN}$ is used as a tracer of PDRs (Greaves \& Church 1996; Rodriguez-Franco et al. 1998; Boger \& Sternberg 2006). We observe $\mathrm{HCN} / \mathrm{CN}$ ratios slightly lower 

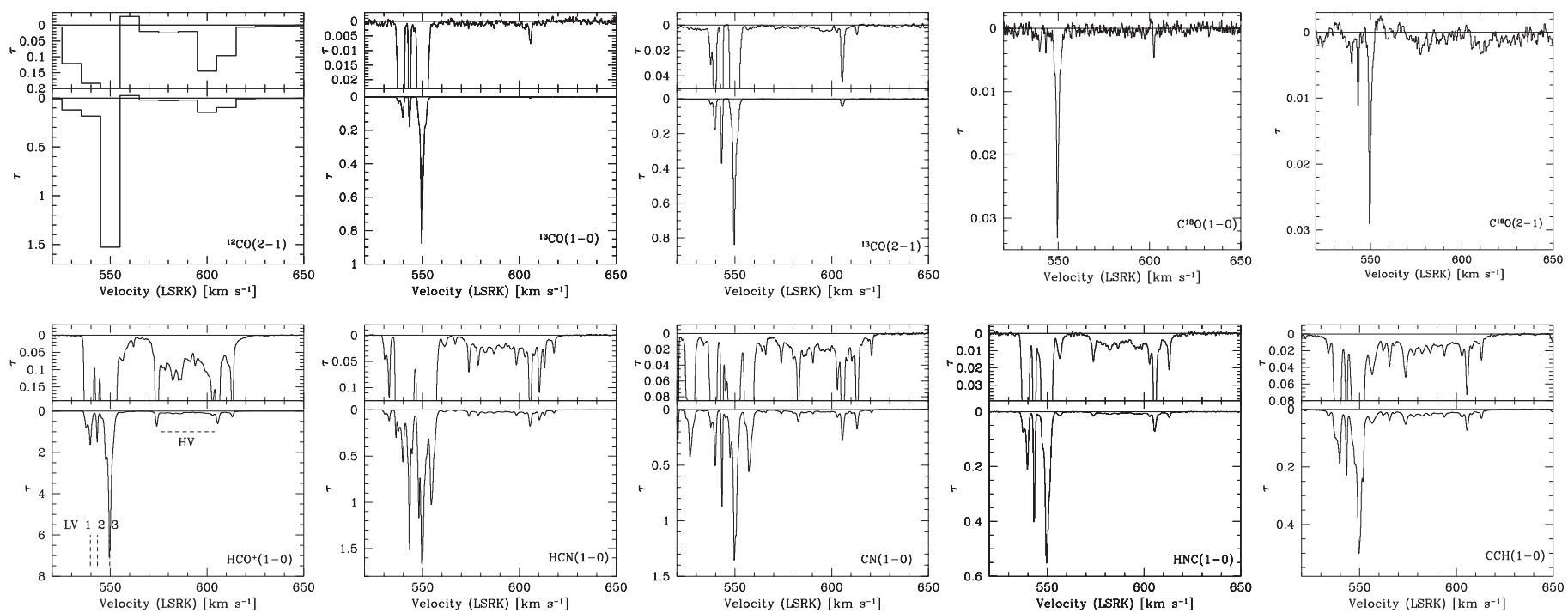

Figure 15. Absorption spectra taken toward the core of Cen A. The ordering of the top and bottom rows are the same as in Figures 7 through 14 . For all transitions except the two $\mathrm{C}^{18} \mathrm{O}$ lines, a second panel above the full spectrum focuses on the lower level high velocity absorption component and the spectral noise. $\mathrm{HCN}(1-0)$, $\mathrm{CN}(1-0)$, and $\mathrm{CCH}(1-0)$ all have hyperfine structure, but we only consider the peak of the main hyperfine component.

in the CND than the arms, with values for the CND of $\sim 2-2.5$, consistent with expected values that are found toward PDRs. $\mathrm{XDR}$ gas is also predicted to be highly elevated in $\mathrm{CN}$ (Meijerink et al. 2006) because XDRs set up PDR-like conditions throughout the bulk of the cloud, not just at its surface. So if XDRs dominated, we would expect an even lower $\mathrm{HCN} / \mathrm{CN}$ ratio. We conclude that while the observed $\mathrm{HCN} / \mathrm{CN}$ ratio demonstrates the presence of pronounced photodissociation, it argues in favor of PDRs or soft XDRs over intense XDRs.

$\mathrm{CCH}$ is also present in PDR gas and is detected in the CND. $\mathrm{CCH}$ is typically found near high radiation environments (e.g., Meier \& Turner 2005; Garcia-Burillo et al. 2017), and although weakly detected in the CND, it could locate a region of high far-UV radiation in the southeastern side of the CND.

In summary, we cannot unambiguously determine the nature of the ionization in the CND based on these line ratios, but we can conclude that the CND gas is denser and more strongly irradiated than the molecular arms. The source of this radiation could be coming from either nuclear star formation/supernovae or from (weak) XDRs associated with the AGN. The PDR origin is weakly favored over XDRs based on $\mathrm{CN}$ and $\mathrm{CCH}$.

\subsubsection{The Influence of the AGN on Gas Chemistry}

The proximity of NGC 5128 allows smaller linear distances from the AGN to be probed than studies dealing with the three times more distant NGC 1068 or NGC 1097 at the same angular resolution. This allows studies of similar angular resolution to probe closer to the AGN. The CND has a couple of characteristics that could possibly suggest an interaction with the AGN: (1) There is a decreased abundance of the rare $\mathrm{CO}$ isotopologues in the CND compared to the dense gas tracers or a high excitation shifting the populations of the rare $\mathrm{CO}$ isotopologues to higher rotational transitions (Israel et al. 2014). (2) The dense gas chemistry suggests that the CND has PDR/XDR qualities.

Bright dense gas tracers in the CND demonstrate that there are large amounts of dense gas present (see Section 4.3.1).
Thus the faintness of the rare CO isotopologues in the CND is unlikely to be due to subthermal excitation. However, non-LTE effects could contribute to the missing isotopologues. A possible explanation for this faintness could be isotopeselective photodissociation due to the radiation field of the AGN or star formation. The radiation field could dissociate much of the rare $\mathrm{CO}$ isotopologues throughout the molecular gas in the CND, but not the ${ }^{12} \mathrm{CO}$, because it is much more strongly self-shielded. The fact that the rare $\mathrm{CO}$ isotopologues are seen in the narrow absorption LV complex gas (but not strongly in the broad HV complex absorption gas) implies that there must remain dense cores along the line of sight that have some associated rare $\mathrm{CO}$ isotopologues emission.

Strong radiation likely photodissociates some of the dense gas tracers, particularly $\mathrm{HNC}$ and $\mathrm{HCN}$, though less so $\mathrm{HCO}^{+}$. (This may be another contributor to the somewhat elevated $\mathrm{HCO}^{+}$line relative to $\mathrm{HCN}$ and $\mathrm{HNC}$ [Section 4.2.2].) But $\mathrm{HCN}$ and $\mathrm{HCO}^{+}$can maintain high abundances in PDRs, and have much higher opacities than the rare $\mathrm{CO}$ isotopologues (Figure 15) and therefore may partially self-shield, which potentially explains their presence despite the photodissociation. Anomalously faint ${ }^{13} \mathrm{CO}(1-0)$ seems to be a common feature of the nuclei of numerous galaxies (e.g., Young \& Sanders 1986; Casoli et al. 1992; Aalto et al. 1995, 1997).

Another possible, though much more speculative, explanation for faint rare $\mathrm{CO}$ isotopologues in the $\mathrm{CND}$, is that the gas in the nucleus of Cen $\mathrm{A}$ is the result of accretion of lower metallicity, high $\mathrm{CO}$ isotopic ratio gas from outside Cen A. Lower metallicity gas appears to favor $\mathrm{HCO}^{+}$over $\mathrm{HCN}$ because $[\mathrm{O} / \mathrm{N}]$ increases with decreasing metallicity (e.g., Anderson et al. 2014). However, this does not explain the "normal" galaxy center isotopic ratios implied for the molecular arms, unless the CND and the molecular arms originate in two separate accretion events. With the current data presented here, we do not see strong evidence for this possibility, but cannot rule it out.

There is no significant difference in any of the dense gas emission ratios of the inner peaks (features C- 3 and D-1) to the outer peaks of the CND (B-3 and E-1). This indicates that the 
chemical sphere of influence of the AGN on the molecular gas must span the entirety of the CND or be confined to inside $\lesssim 3^{\prime \prime}$ currently hidden by the absorption toward the AGN. The presence of PDR qualities throughout the CND suggests that either the AGN is actively influencing the entire disk or something else is causing the PDR features. There have been direct observations of influences of an AGN jet on the molecular gas in NGC 1068 (Garcia-Burillo et al. 2014) and M51 (Matsushita et al. 2015; Querejeta et al. 2016), so an interaction in Cen A could be expected. However, Marconi et al. (2000) calculated a maximum star formation rate in the inner kiloparsec of Cen A to be around $1.0 M_{\odot} \mathrm{yr}^{-1}$ based on the $\operatorname{Pa} \alpha$ emission. This value includes regions of the molecular arms that are currently forming stars, so the star formation that would interact primarily with CND is less than $1.0 M_{\odot} \mathrm{yr}^{-1}$. This star formation could result in increased ionization rates and shocks required to explain the observed line ratios. So there might be an influence between the AGN and the molecular gas in the CND, but we cannot rule out star formation or shocks to explain the observed line ratios without a better constraint on the star formation rate in the CND.

\subsection{Mass Analysis}

\subsubsection{Dynamical Masses}

Using the $\mathrm{CND}$ as traced by the $\mathrm{HCO}^{+}(1-0)$ image cube, we estimate the enclosed mass between roughly locations $\mathrm{B}$ and $\mathrm{E}$ and locations $\mathrm{C}$ and D using the equation $M_{\mathrm{enc}}=\frac{v^{2} R}{G \sin ^{2} i}$, where $v$ is projected rotational velocity measured at each location, $R$ is the projected distance to that location from the center of the galaxy, $G$ is the gravitational constant, and $i$ is the inclination of the disk. Spectra were obtained toward these regions, and Gaussians were fit to the spectra in order to determine the mean velocity of the gas at these locations. Locations B and E trace the outer regions of the CND and probe gas at a radius of $145 \pm 15 \mathrm{pc}$ from the center of the disk, while locations $\mathrm{C}$ and $\mathrm{D}$ are the inner peaks of the CND and probe gas at a radius of about $65 \pm 15 \mathrm{pc}$ from the center. We measured a velocity difference of $355 \pm 20 \mathrm{~km} \mathrm{~s}^{-1}$ between $\mathrm{B}$ and $\mathrm{E}$ and $185 \pm$ $20 \mathrm{~km} \mathrm{~s}^{-1}$ between $\mathrm{C}$ and $\mathrm{D}$. We use the reported inclination angle of $71^{\circ}$ in Espada et al. (2009) and Quillen et al. (2010) for the outer region of the CND (B and E) to determine an enclosed dynamical mass of about $1.4 \pm 0.2 \times 10^{9} M_{\odot}$. The uncertainty arises mainly from uncertainties in the inclination angle of about $\pm 10^{\circ}$. For the inner portion of the CND (C and D), we use the reported value of $37^{\circ}$ for the inclination angle from Quillen et al. (2010) to determine an enclosed dynamical mass of $3.6 \times 10^{8} M_{\odot}$ (uncertain by a factor of 2 ) within the inner $130 \mathrm{pc}$ of the CND. These values are greater than the mass of the SMBH $\left(5.5 \times 10^{7} M_{\odot}\right.$, Cappellari et al. 2009) by factors of 25 for the entire CND and about 6 between the inner peaks of the CND. Thus the SMBH only begins to dominate the mass budget on scales much smaller than $\mathrm{R} \sim 50 \mathrm{pc}$.

\subsubsection{Conversion Factor Analysis}

The column density of $\mathrm{H}_{2}, \mathrm{~N}_{\mathrm{H}_{2}}$, can be calculated by using a conversion factor $\mathrm{X}$ between the ${ }^{12} \mathrm{CO}(1-0)$ intensity and $\mathrm{N}_{\mathrm{H}_{2}}$. We can estimate the $\mathrm{N}_{\mathrm{H}_{2}}$ from the ${ }^{12} \mathrm{CO}(2-1)$ science verification data by assuming a ratio of ${ }^{12} \mathrm{CO}(2-1)$ to ${ }^{12} \mathrm{CO}(1-0)$ of 0.8 (Leroy et al. 2009), as both transitions are optically thick. If we adopt the standard Galactic disk conversion factor of $X=2.0 \times 10^{20} \mathrm{~cm}^{-2}\left(\mathrm{~K} \mathrm{~km} \mathrm{~s}^{-1}\right)^{-1}$
(Strong et al. 1988; Hunter et al. 1997; Bolatto et al. 2003), an $\mathrm{H}_{2}$ mass of $1.2 \times 10^{8} M_{\odot}$ is calculated for the CND region. This value was obtained from the integrated intensity map of the ${ }^{12} \mathrm{CO}(2-1)$ transition of the inner 500 pc of NGC 5128, which includes both the molecular arm and the CND emission, so this is likely an upper limit on the $\mathrm{H}_{2}$ mass in the CND. This $\mathrm{H}_{2}$ mass is about $10 \%$ of the residual mass between the calculated dynamical mass of the CND and $M_{\mathrm{SMBH}}$. Using the conversion factor for more energetic environments from Downes \& Solomon $\left(1998 ; X=0.4 \times 10^{20} \mathrm{~cm}^{-2}[\mathrm{~K} \mathrm{~km}\right.$ $\mathrm{s}^{-1]-1}$ ), we calculate the $\mathrm{H}_{2}$ mass to be $2.4 \times 10^{7} M_{\odot}$, which is about $2 \%$ of the residual mass between the dynamical mass and the supermassive black hole. Israel et al. (2014) use LVG and PDR models to determine the conversion factor for the CND in NGC 5128 to be $X=4.0 \times 10^{20} \mathrm{~cm}^{-2}\left(\mathrm{~K} \mathrm{~km} \mathrm{~s}^{-1}\right)^{-1}$. Using this value, we estimate the $\mathrm{H}_{2}$ mass to be $2.4 \times 10^{8} M_{\odot}$, which is about $20 \%$ of the difference between the calculated dynamical and the SMBH mass.

The $\mathrm{H}_{2}$ column can also be calculated from the ${ }^{13} \mathrm{CO}$ data if the excitation temperature, opacities, and abundance ratios are known. The temperature can be determined using the $J=2-1$ and $J=1-0$ rotational transitions of ${ }^{13} \mathrm{CO}$, and the opacities and abundance ratios are determined through isotopologue analysis. For the CND, where we do not detect ${ }^{13} \mathrm{CO}(1-0)$, we get a limit on the column. If we adopt an excitation temperature for the CND of $20 \mathrm{~K}$ (Section 4.2.1), and use an abundance ratio of $\left[{ }^{12} \mathrm{CO} /{ }^{13} \mathrm{CO}\right]=50$ and $\left[{ }^{12} \mathrm{CO} / \mathrm{H}_{2}\right]=8.5 \times 10^{-5}$ (Frerking et al. 1982), a total gas mass of $\sim 2 \times 10^{6} M_{\odot}$ for the CND is calculated. This value is lower by a factor of $\gtrsim 10$ than the gas masses calculated using ${ }^{12} \mathrm{CO}(2-1)$ and any of the previously noted conversion factors. This discrepancy could be due to one or several reasons including our adopted $\left[{ }^{12} \mathrm{CO} /{ }^{13} \mathrm{CO}\right]$ abundance ratio could be incorrect (isotope-selective photodissociation may play an important role; see Section 4.2.3), the line excitation is much different from $20 \mathrm{~K}$, or the conversion factor may be much lower.

If we consider the molecular arm features individually, we can estimate the $\mathrm{H}_{2}$ column toward spectral features A-1, B-1, F-2, G-2, and H-1. From the isotopologue analysis, the temperatures of the molecular arm features are around $10 \mathrm{~K}$ for features A-1, B-1, F-2, and H-1, but more than $20 \mathrm{~K}$ for G-2. We find that the $\mathrm{H}_{2}$ column is also universally lower when calculated from ${ }^{13} \mathrm{CO}(2-1)$ than from ${ }^{12} \mathrm{CO}(2-1)$ toward the molecular arms. For the spectral features A-1, F-2, G-2, and $\mathrm{H}-1$, the calculated values are only a factor of about 2 lower, which is likely within the uncertainties of the analysis. However, for region $\mathrm{B}-1$ the ${ }^{13} \mathrm{CO}(2-1)$ determined $\mathrm{H}_{2}$ column is lower than the ${ }^{12} \mathrm{CO}(2-1)$ calculated value by around 20, suggesting there are different physical conditions (excitation and opacity) for this feature compared to the rest of the molecular arm components.

\subsection{Comparing Absorption and Emission}

Our observations include absorption as well as emission. A detailed analysis of the individual absorption components and their chemistry will be discussed in a forthcoming paper. Here we focus on the line ratios in absorption that directly confront the emission in order to address which emission component is most likely responsible for the absorption features. Absorption depth is converted to line opacity (Section 3.4.2); then optical depth ratios are calculated for comparison with the emission 
Table 4

Opacity Ratios for Selected Absorption Components

\begin{tabular}{|c|c|c|c|c|}
\hline Ratio & $\begin{array}{l}\text { Abs. Comp. } 1 \\
\left(539.7 \mathrm{~km} \mathrm{~s}^{-1}\right)\end{array}$ & $\begin{array}{l}\text { Abs. Comp. } 2 \\
\left(543.4 \mathrm{~km} \mathrm{~s}^{-1}\right)\end{array}$ & $\begin{array}{l}\text { Abs. Comp. } 3 \\
\left(549.7 \mathrm{~km} \mathrm{~s}^{-1}\right)\end{array}$ & $\begin{array}{l}\text { Broad Median } \\
\left(576-604 \mathrm{~km} \mathrm{~s}^{-1}\right)\end{array}$ \\
\hline$\frac{\frac{{ }^{12} \mathrm{CO}(2-1)}{{ }^{13} \mathrm{CO}(2-1)}}{4}$ & $1.05^{\mathrm{a}} \pm 0.01$ & $0.50^{\mathrm{a}} \pm 0.01$ & $1.82 \pm 0.02$ & $\sim 12.5$ \\
\hline$\frac{{ }^{13} \mathrm{CO}(2-1)}{{ }^{13} \mathrm{CO}(1-0)}$ & $1.45 \pm 0.01$ & $2.08 \pm 0.02$ & $1.01 \pm 0.01$ & $>2.5$ \\
\hline$\frac{{ }^{12} \mathrm{CO}(2-1)}{\mathrm{C}^{18} \mathrm{O}(2-1)}$ & $46^{a} \pm 8$ & $17^{\mathrm{a}} \pm 1$ & $53 \pm 2$ & $>31$ \\
\hline$\frac{{ }^{13} \mathrm{CO}(2-1)}{\mathrm{C}^{18} \mathrm{O}(2-1)}$ & $44 \pm 8$ & $34 \pm 2$ & $29 \pm 1$ & $>2.5$ \\
\hline$\frac{{ }^{12} \mathrm{CO}(2-1)}{\mathrm{HCO}^{+}(1-0)}$ & $0.11^{\mathrm{a}} \pm 0.01$ & $0.14^{\mathrm{a}} \pm 0.01$ & $0.22 \pm 0.01$ & $0.27 \pm 0.01$ \\
\hline$\frac{{ }^{13} \mathrm{CO}(1-0)}{\mathrm{HCN}(1-0)}$ & $0.22 \pm 0.01$ & $0.12 \pm 0.01$ & $0.50 \pm 0.01$ & $<0.04$ \\
\hline$\frac{\mathrm{HCN}(1-0)}{\mathrm{HCO}^{+}(1-0)}$ & $0.34 \pm 0.01$ & $1.17^{\mathrm{a}} \pm 0.01$ & $0.24^{\mathrm{a}} \pm 0.01$ & $0.21^{\mathrm{a}} \pm 0.01$ \\
\hline$\frac{\mathrm{HCN}(1-0)}{\mathrm{HNC}(1-0)}$ & $2.89 \pm 0.01$ & $3.77^{\mathrm{a}} \pm 0.04$ & $3.00^{\mathrm{a}} \pm 0.03$ & $3.3^{\mathrm{a}} \pm 0.3$ \\
\hline$\frac{\mathrm{HCN}(1-0)}{\mathrm{CN}(1-0)}$ & $1.11 \pm 0.01$ & $1.74^{\mathrm{a}} \pm 0.02$ & $1.23^{\mathrm{a}} \pm 0.01$ & $0.71^{\mathrm{a}} \pm 0.03$ \\
\hline$\frac{\mathrm{HCN}(1-0)}{\mathrm{CCH}(1-0)}$ & $3.00 \pm 0.01$ & $6.56^{\mathrm{a}} \pm 0.07$ & $3.32^{\mathrm{a}} \pm 0.03$ & $1.54^{\mathrm{a}} \pm 0.08$ \\
\hline$\frac{\mathrm{CCH}(1-0)}{\mathrm{CN}(1-0)}$ & $0.37 \pm 0.01$ & $0.26^{\mathrm{a}} \pm 0.01$ & $0.37^{\mathrm{a}} \pm 0.01$ & $0.46^{\mathrm{a}} \pm 0.02$ \\
\hline
\end{tabular}

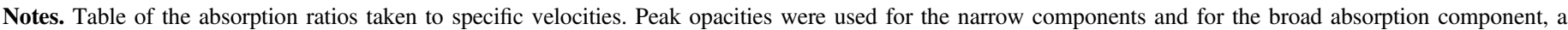

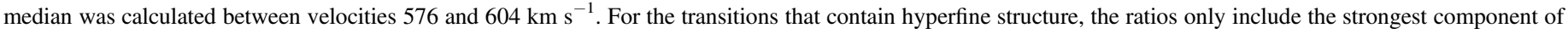
the hyperfine.

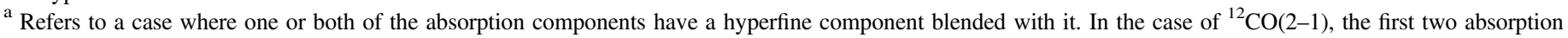
components are blended together due to poor velocity resolution of the data.

line ratios. As long as temperature/excitation does not change significantly between the two transitions making up the ratios and assuming the transitions are optically thin, then the $\tau$ absorption ratio and the $\mathrm{T}$ emission ratios may be compared robustly. Hence if the emission ratios and the absorption ratios are similar, this indicates the same gas component is being sampled.

Ratios of the calculated optical depth $\tau$ were taken from the absorption spectra shown in Figure 15 and are reported in Table 4. Three deep absorption lines in the low velocity complex (LV complex), located at 539.7, 543.4, and $549.7 \mathrm{~km} \mathrm{~s}^{-1}$, and the median of the spectra from velocities $576-604 \mathrm{~km} \mathrm{~s}^{-1}$ to represent the broad, high velocity absorption complex (HV complex) are discussed. The opacity ratio $\tau\left[{ }^{13} \mathrm{CO}(2-1)\right] /$ $\tau\left[{ }^{13} \mathrm{CO}(1-0)\right]$ ranges from about 1 to 2 in the absorption, and the emission intensity ratio ranges from 1 to 2 in the molecular arms, but the non-detection of ${ }^{13} \mathrm{CO}(1-0)$ in the CND puts the opacity ratio at a lower limit of about 2 (emission intensity ratios taken from Table 3).

The opacity ratio of $\tau\left[{ }^{13} \mathrm{CO}(2-1)\right] / \tau\left[\mathrm{C}^{18} \mathrm{O}(2-1)\right]$ is around 30 in the narrow LV complex components of the absorption and $>2.5$ in the HV complex (Table 4). In emission, this ratio ranges from about 4 to 20 in the molecular arms and a lower limit of 4 in the CND (Table 3). For spectral features B-1 and $\mathrm{G}-2$, the optical depth ratios from the isotopologue analysis would be $\sim 20$. Based on this $\mathrm{CO}$ isotopologue ratio, the narrow LV complex could be associated with the molecular arms and the HV complex with the CND, but this conclusion remains ambiguous from $\mathrm{CO}$ isotopologues alone and runs into problems when considering the dense gas tracers.

The ${ }^{13} \mathrm{CO}(1-0) / \mathrm{HCN}(1-0)$ optical depth ratio is very low in all absorption cases, ranging from 0.04 in the $\mathrm{HV}$ complex to about $0.1-0.5$ in the narrow LV complex lines. The emission components from the molecular arms all have an emission ratio greater than about 3.5 for ${ }^{13} \mathrm{CO}(1-0) / \mathrm{HCN}(1-0)$, while the ratio for the CND is an upper limit around 0.2 for the outer peaks and 0.1 for the inner peaks.

The single dish data of Israel et al. (2014) have beam sizes of $47^{\prime \prime}$ and $57^{\prime \prime}$ for ${ }^{13} \mathrm{CO}(1-0)$ and $\mathrm{HCN}(1-0)$, respectively. Assuming emission is uniform over the $\mathrm{HCN}$ beam, the ratio is 4.6. If the emission is more compact, this ratio could be as low as 3 . This suggests that there is $\lesssim 25 \%$ extended emission that is not recovered by our interferometric ALMA observations. Therefore we consider the uncertainties of ratios between diffuse $\mathrm{CO}$ and the more concentrated high density tracers to be at most $30 \%$. Ratios between different $\mathrm{CO}$ transitions or between dense gas tracers should be more certain than this.

There is a strong bimodality between the CND and the molecular arms in the ${ }^{13} \mathrm{CO}(1-0) / \mathrm{HCN}(1-0)$ emission ratio, with values of less than 0.2 for the CND and values of 3 or larger for the molecular arms. All of the other ratios seem to be roughly consistent between the two gas regions (Tables 3 and 4). So based on the ${ }^{13} \mathrm{CO}(1-0) / \mathrm{HCN}(1-0)$ ratio, the ratios of the opacities in the LV absorption components follow the CND values more closely than the corresponding values for the 
molecular arms, suggesting that the gas in the LV complex absorption is also more likely related to the CND rather than the molecular arms. However, there is an important caveat-namely, absorption is sensitive to molecules in the $J=0$ state while emission is sensitive to gas in the $J=1$ state. Therefore, it is possible that if there is a large amount of very cold/subthermal gas, then the $\mathrm{HCN}$ absorption will be significantly elevated relative to ${ }^{13} \mathrm{CO}$. If this is the case, then it may still be possible for the absorption components to result from molecular gas farther out than the CND. However, this effect should not significantly affect the comparison of the ratios between the dense gas tracers.

The absorption component centered on roughly $543 \mathrm{~km} \mathrm{~s}^{-1}$ seems to be different from the other two narrow LV complex components in a number of ratios. This component has a much narrower line width than the other two and is the only region in both absorption or emission, where $\mathrm{HCN}(1-0)$ is stronger than $\mathrm{HCO}^{+}(1-0)$. The differences in the ratios of the other dense gas tracers for this component suggest that $\mathrm{HCN}(1-0)$ is enhanced compared to the other narrow LV complex components. The pencil beam for the absorption could be picking out a specific region of a molecular cloud that happens to deviate from the average that is observed in emission.

The optical depth ratio of $\mathrm{HCN}(1-0) / \mathrm{CN}(1-0)$ toward the narrow LV components are 1.1-1.7, which is similar to the CND emission values of 1.7 (C-3). Likewise for the $\mathrm{HCN}(1-0) / \mathrm{CCH}$ (1-0) opacity ratios, the narrow LV components are 3.0-6.6, consistent with the emission value of 3.9. Therefore these ratios also suggest that the narrow line LV components could be associated with the CND. The $\mathrm{HV}$ component is further enhanced in $\mathrm{CN}$ and $\mathrm{CCH}$ relative to the $\mathrm{CND} / \mathrm{LV}$ $(\mathrm{HV}: \mathrm{HCN}(1-0) / \mathrm{CN}(1-0) \simeq 0.7$ and $\mathrm{HCN}(1-0) / \mathrm{CCH}(1-0) \simeq$ 1.5). $\mathrm{CN}$ and $\mathrm{CCH}$ are both molecules that are present in PDR/ XDRs, which indicates that the HV complex component is located in a region of stronger radiation field, possibly closer to the AGN.

Combining this with the previous statement that the gas seen in absorption seems to be similar to the gas that is observed in the CND suggests the absorption features may be placed entirely in the CND. The narrow LV complex components, which are at velocities much closer to systemic, must be coming from gas crossing in front of the AGN with small noncircular motions. The redshifted HV complex component of absorption then would come from gas much closer to the AGN. In fact, Espada et al. (2010) used VLBA observations of H I to determine that the broad component is very close to the AGN by finding a significant change in the $\mathrm{HV}$ complex on scales of 0.4 pc. Muller et al. (2009) do not observe $\mathrm{HCO}^{+}(4-3)$ or $\mathrm{HCN}(4-3)$ in the HV complex and constrain the density of the LV absorption gas to be a few $10^{4} \mathrm{~cm}^{-3}$, consistent with the gas chemistry measured in the CND.

\subsection{The Warped Disk Model}

Quillen et al. (2010) have compiled a warped disk model of NGC 5128 that ranges from 2 pc up to 6500 pc using $2.122 \mu \mathrm{m}$ molecular hydrogen line data (inner $3^{\prime \prime}$; Neumayer et al. 2007), SMA CO observations (inner 1'; Espada et al. 2009), Spitzer $8 \mu \mathrm{m}$ ( $>40^{\prime \prime}$; Quillen et al. 2006), and H I observations ( $>60^{\prime \prime}$; Struve et al. 2010). We do not detect the entirety of the dust lane, so we do not compare our data to the large scale portions of the model. We do detect the entire CND, which is about $22^{\prime \prime} \times 11^{\prime \prime}$ in size at a position angle of $155^{\circ}$ and an inclination of about $60^{\circ}$ for the outer components compared to the position angle of $150^{\circ}$ and inclination of $\sim 70^{\circ}$ reported in Quillen et al. (2010). The inner components of the CND are at a radius of about 65 pc from the AGN.

Using the warped disk model from Quillen et al. (2010), we develop a potential picture of the inner region of Cen A. The inner $200 \mathrm{pc}$ of NGC 5128 contains the CND, which is abundant in dense gas. Beyond the CND, there appears to be a gap in the molecular gas, before reaching the warped rings that create the parallel molecular arms, seen prominently in $\mathrm{CO}$ and its rare isotopologues. These warps create a ridge of overlapping molecular gas in the southeastern and northwestern regions explaining the molecular arms. The front and back sides of the turnover in the warp could possibly explain the splitting along the arms seen in the PV diagrams (see Figure 6, Slice 3). The regions where fewer of the molecular rings overlap are too faint to be detected by our observations.

The jet from the AGN is roughly perpendicular to the outer part of the CND, and we assume that the northern jet is approaching and is brighter due to Doppler boosting (Jones et al. 1996; Tingay et al. 1998; see also Figure 1). This would mean that the CND, which is at a position angle of roughly $150^{\circ}$ and inclined at about $60^{\circ}$, has the northeastern side of the disk oriented away from Earth and the southwestern side closer to Earth. This would put the redshifted side of the CND (the northwestern side), which have velocities of $\geqslant 580 \mathrm{~km} \mathrm{~s}^{-1}$, behind the AGN meaning that the HV complex component in the absorption cannot be coming from the redshifted side of the CND, assuming the CND is purely flat with only circular motions. However, from comparing the chemistry in the absorption to the emission components, the LV and HV absorption components appear more similar to the CND than the molecular arms.

Based on our data, we present two different scenarios that could result in the observed absorption and emission. In the first scenario, all of the narrow LV complex components of the absorption come from where individual clouds in the CND cross the AGN. The broad component would then come from material closer to the middle of the CND that is falling in toward the AGN, which may ultimately fuel the jet. If the gas is falling in toward the black hole, one would expect that the gas would be stretched in velocity space, as the gas nearer to the black hole is moving at a higher velocity than gas that is farther away. This could be consistent with the HV complex being similar to one of the narrow components but stretched and redshifted over a range of $25-60 \mathrm{~km} \mathrm{~s}^{-1}$ as the gas falls in toward the black hole. Eckart et al. (1999) described a scenario in which both of the LV and HV complexes originate from gas farther away from the AGN than the CND; however, based on the chemistry of the absorption components, the gas in absorption appears to be more closely related to the CND than gas farther away.

In the second scenario, the warps in the molecular gas of Cen A continue into the innermost part of the CND, and it is possible that the warps are severe enough to place part of the redshifted side of the CND in front of the AGN to create the broad HV complex component, while some other non-warped parts of the CND remain in front to make the narrow roughly systemic LV complex components.

The velocities of the inner peaks of the CND are at about 470 and $660 \mathrm{~km} \mathrm{~s}^{-1}$ and at a radius of about $65 \mathrm{pc}$, which means that the rotational velocity of that tilted ring is $1 / 2$ the 
difference of those velocities, around $95 \mathrm{~km} \mathrm{~s}^{-1}$. The maximum redshifted velocity that we observe in the absorption is about $60 \mathrm{~km} \mathrm{~s}^{-1}$ off the systemic velocity, meaning that gas interior to the inner peaks of the CND would be responsible for parts of the HV complex component. However, this gas would need an extreme warp for these high velocities, so noncircular orbits are likely present. In fact, large noncircular motions are found in the CND by Espada et al. (2017). The chemistry of the $\mathrm{HV}$ complex component is enhanced in the PDR/XDR tracers $\mathrm{CN}$ and $\mathrm{CCH}$. This would be expected of gas that is getting closer to the radiation field from the AGN. This is also consistent with the fact that we detect $\mathrm{CN}$ emission almost exclusively from the inner CND.

\section{Summary}

We observed various molecular transitions toward the inner 500 pc of Centaurus A with ALMA and obtained maps and spectra at much higher sensitivity than previous observations. Based on these observations and analysis performed, we draw the following conclusions about the nuclear region of Cen A:

1. The molecular arms are kinematically and chemically distinct from the CND. The molecular arms are strongly detected in $\mathrm{CO}$ and its isotopologues but dense gas tracers like $\mathrm{HCO}^{+}$and $\mathrm{HCN}$ were not detected or detected at only very weak levels. The CND, however, is only weakly detected in the rare $\mathrm{CO}$ isotopologues, but it is strongly detected in the dense gas tracers. There is no apparent connection between the emission components corresponding to the molecular arms and the CND. However, the lack of short baselines and resulting lack of sensitivity to large scale structure may have meant a connection between the components was not detected. Without a clear transition between the molecular arms and the CND, the mechanism for the transportation of molecular gas from the outer galaxy to the nuclear is still missing.

2. Both the high and low velocity complexes in the absorption profiles appear to originate from gas in the CND. The optical depth ratios from the absorption in both the high and low velocity complexes match the line ratios from the $\mathrm{CND}$ and not the dust lane. Also, the dense gas tracers that are prominent in the CND, but weak or not detected in the molecular arms, were also prominent in absorption. Placing all of the absorption profiles within the CND means that the velocities from the absorption profiles must be accounted for by the kinematics of the CND.

3. The high velocity complex of the absorption spectra comes from gas that may be infalling toward the AGN. With all of the absorption components placed inside the CND, the high velocity offset of the high velocity complex could be the result of the gas falling in toward the AGN. If this gas is indeed infalling toward the AGN, then it could potentially be the gas that will fuel the central engine that is powering the radio jet. The HV complex has long been considered as potentially infalling gas based on prior observations of the absorption (e.g., Gardner \& Whiteoak 1976; van der Hulst et al. 1983; Seaquist \& Bell 1990; Israel et al. 1991).

4. The AGN may be radiatively influencing the CND. The lack of emission in low- $J$ level transitions of the rare $\mathrm{CO}$ isotopologues and bright $\mathrm{CN}$ and $\mathrm{CCH}$ in the inner CND suggest that energy is being injected into the CND. The poorly constrained level of star formation in the CND suggests the AGN as a possible source of the radiation. If the $\mathrm{AGN}$ is indeed responsible for the energy input to the CND, this would be direct chemical feedback from the AGN to the molecular gas in the nuclear region of the galaxy.

We thank the referee for comments that improved the manuscript. We would like to thank Fabian Walter for stimulating conversations and comments. D.E. was supported by JSPS KAKENHI Grant Number JP 17K14254. This manuscript makes use of the following ALMA data: ADS/JAO.ALMA \#2011. 0.00008.SV and 2011.0.00010.S. The National Radio Astronomy Observatory is a facility of the National Science Foundation operated under cooperative agreement by Associated Universities, Inc. ALMA is a partnership of ESO (representing its member states), NSF (USA), and NINS (Japan), together with NRC (Canada), NSC and ASIAA (Taiwan), and KASI (Republic of Korea), in cooperation with the Republic of Chile. The Joint ALMA Observatory is operated by ESO, AUI/NRAO, and NAOJ. M.M. and D.S.M. acknowledge support from National Science Foundation Grant AST-1109803. M.M. is a student at the National Radio Astronomy Observatory.

\section{ORCID iDs}

Jürgen Ott (i) https://orcid.org/0000-0001-8224-1956

David S. Meier (iD https://orcid.org/0000-0001-9436-9471

Sébastien Muller (iD https://orcid.org/0000-0002-9931-1313

Daniel Espada (i) https://orcid.org/0000-0002-8726-7685

Sergio Martín (iD https://orcid.org/0000-0001-9281-2919

Frank P. Israel (iD https://orcid.org/0000-0002-6760-9449

Christian Henkel (i) https://orcid.org/0000-0002-7495-4005

Andreas Brunthaler (i) https://orcid.org/0000-0003-

4468-761X

Nadine Neumayer (iD https://orcid.org/0000-0002-6922-2598

Alison B. Peck (iD https://orcid.org/0000-0001-8276-0000

\section{References}

Aalto, S. 2008, Ap\&SS, 313, 273

Aalto, S., Booth, R. S., Black, J. H., et al. 1995, A\&A, 300, 369

Aalto, S., Polatidis, A. G., Hüttemeister, S., et al. 2002, A\&A, 381, 783

Aalto, S., Radford, S. J. E., Scoville, N. Z., et al. 1997, ApJL, 475, L107

Aguado, A., Roncero, O., Zanchet, A., et al. 2017, ApJ, 838, 33

Anderson, C., Meier, D., Ott, J., et al. 2014, ApJ, 793, 37

Auld, R., Smith, M. W. L., Bendo, G., et al. 2012, MNRAS, 420, 1882

Baan, W. A., Henkel, C., Loenen, A. F., et al. 2008, A\&A, 477, 747

Boger, G. I., \& Sternberg, A. 2006, ApJ, 645, 314

Bolatto, A. D., Wolfire, M., \& Leroy, A. K. 2003, ARA\&A, 51, 207

Cappellari, M., Neumayer, N., Reunanen, J., et al. 2009, MNRAS, 394, 660

Casoli, F., Dupraz, C., \& Combes, F. 1992, A\&A, 264, 55

Charmandaris, V., Combes, F., \& van der Hulst, J. M. 2000, A\&A, 356, L1

Clarke, D. A., Burns, J. O., \& Norman, M. L. 1992, ApJ, 395, 444

Condon, J. J. 1984, ApJ, 287, 461

Crockett, R. M., Shabala, S. S., Kaviraj, S., et al. 2012, MNRAS, 421, 1603

Davis, T. A., Alatalo, K., Sarzi, M., et al. 2011, MNRAS, 417, 882

Downes, D., \& Solomon, P. M. 1998, ApJ, 507, 615

Eckart, A., Cameron, M., Rothermel, H., et al. 1990, ApJ, 363, 451

Eckart, A., Wild, W., \& Ageorges, N. 1999, ApJ, 516, 769

Espada, D., Matsushita, S., Miura, R., et al. 2017, ApJ, 843, 136

Espada, D., Matsushita, S., Peck, A. B., et al. 2009, ApJ, 695, 116

Espada, D., Matsushita, S., Peck, A. B., et al. 2012, ApJL, 756, L10

Espada, D., Peck, A. B., Matsushita, S., et al. 2010, ApJ, 720, 666

Espada, D., Verdes-Montenegro, L., Huchtmeier, W., et al. 2011, A\&A, 532,117 
Feain, I. J., Cornwell, T. J., Ekers, R. D., et al. 2011, ApJ, 740, 11 Flower, D. R. 1999, MNRAS, 305, 651

Frerking, M. A., Langer, W. D., \& Wilson, R. W. 1982, ApJ, 262, 590

Garcia-Burillo, S., Combes, F., Usero, A., et al. 2014, A\&A, 567, A125

Garcia-Burillo, S., Viti, S., Combes, F., et al. 2017, arXiv:1709.05895

Gardner, F., \& Whiteoak, J. 1976, PASA, 3, 63

Goldsmith, P. F., \& Kauffmann, J. 2017, ApJ, 841, 25

Greaves, J. S., \& Church, S. E. 1996, MNRAS, 283, 1179

Green, S., \& Thaddeus, P. 1974, ApJ, 191, 653

Harada, N., Herbst, E., \& Wakelam, V. ApJ, 721, 1570

Hardcastle, M. J., Worrall, D. M., Kraft, R. P., et al. 2003, ApJ, 593, 169

Häring-Neumayer, N., Cappellari, M., Rix, H.-W., et al. 2006, ApJ, 643, 226

Harris, G. L. H., Rejkuba, M., \& Harris, W. E. 2010, PASA, 27, 457

Hawarden, T. G., Sandell, G., Matthews, H. E., et al. 1993, MNRAS, 260, 844

Henkel, C., Asiri, H., Ao, Y., et al. 2014, A\&A, 565, A3

Henkel, C., Mauersberger, R., Wiklind, T., et al. 1993, A\&A, 268, 17

Hunter, S. D., Bertsch, D. L., Catelli, J. R., et al. 1997, ApJ, 481, 205

Israel, F. P. 1992, A\&A, 265, 487

Israel, F. P. 1998, A\&ARv, 8, 237

Israel, F. P., Güsten, R., Meijerink, R., et al. 2014, A\&A, 562, A96

Israel, F. P., Raban, D., Booth, R. S., et al. 2008, A\&A, 483, 741

Israel, F. P., van Dishoeck, E. F., Baas, F., et al. 1990, A\&A, 227, 342

Israel, F. P., van Dishoeck, E. F., Baas, F., et al. 1991, A\&A, 245, 13

Izumi, T., Kohno, K., Aalto, S., et al. 2013, PASJ, 65, 100

Izumi, T., Kohno, K., Aalto, S., et al. 2016, ApJ, 818, 42

Jones, D., Tingay, S., Murphy, D., et al. 1996, ApJ, 466L, 63

Kainulainen, J. T., Alves, J. F., Beletsky, Y., et al. 2009, A\&A, 502, 5

Kohno, K. 2003, in ASP Conf. Ser. 289, The Proc. IAU 8th Asian-Pacific Regional Meeting 1, ed. S. Ikeuchi, J. Hearnshaw, \& T. Hanawa (San Francisco, CA: ASP), 349

Krips, M., Neri, R., García-Burillo, S., et al. 2008, ApJ, 677, 262

Leeuw, L. L., Hawarden, T. G., Matthews, H. E., et al. 2002, ApJ, 565, 131

Leon, S., Lim, J., Combes, F., et al. 2003, in ASP Conf. Ser. 290, Active Galactic Nuclei: from Central Engine to Host Galaxy, ed. S. Collin, F. Combes, \& I. Shlosman (San Francisco, CA: ASP), 525

Leroy, A. K., Walter, F., Bigiel, F., et al. 2009, AJ, 137, 4670

Lim, J., Leon, S., Combes, F., et al. 2003, in ASP Conf. Ser. 290, Active Galactic Nuclei: from Central Engine to Host Galaxy, ed. S. Collin, F. Combes, \& I. Shlosman (San Francisco, CA: ASP), 529

Lique, F., Spielfiedel, A., Feautrier, N., et al. 2010, JChPh, 132, 024303

Liszt, H. 2001, A\&A, 371, 865

Loenen, A., Spaans, M., Baan, W., \& Meijerink, R. 2008, A\&A, 488, L5

Lovas, F. J., \& Dragoset, R. A. 2004, NIST Recommended Rest Frequencies for Observed Interstellar Molecular Microwave Transitions-2002 Revision, (version 2.0.1), (Online) Available: http://physics.nist.gov/ restfreq [2009, February 4]. National Institute of Standards and Technology, Gaithersburg, MD

Malin, D. F., Quinn, P. J., \& Graham, J. A. 1983, ApJL, 272, L5

Marconi, A., Capetti, A., Axon, D. J., et al. 2001, ApJ, 549, 915

Marconi, A., Pastorini, G., Pacini, F., et al. 2006, A\&A, 448, 921
Marconi, A., Schreier, E. J., Koekemoer, A., et al. 2000, ApJ, 528, 276

Martín, S., Kohno, K., Izumi, T., et al. 2015, A\&A, 573, A116

Matsushita, S., Trung, D.-V., Boone, F., et al. 2015, PKAS, 30, 439

McMullin, J. P., Waters, B., Schiebel, D., Young, W., \& Golap, K. 2007, in ASP Conf. Ser. 376, Astronomical Data Analysis Software and Systems XVI, ed. R. A. Shaw, F. Hill, \& D. J. Bell (San Francisco, CA: ASP), 127

Meier, D. S., \& Turner, J. L. 2000, BAAS, 32, 1455

Meier, D. S., \& Turner, J. L. 2005, ApJ, 618, 259

Meijerink, R., Spaans, M., \& Israel, F. P. 2006, ApJ, 650L, 103

Meijerink, R., Spaans, M., \& Israel, F. P. 2007, A\&A, 461, 793

Meijerink, R., Spaans, M., Loenen, A. F., et al. 2011, A\&A, 525, 119

Mirabel, I. F., Laurent, O., Sanders, D. B., et al. 1999, A\&A, 341, 667

Muller, N., Sébastien, N., \& Dinh-V-Trung, N. 2009, ApJ, 696, 176

Neumayer, N. 2010, PASA, 27, 449

Neumayer, N., Cappellari, M., Reunanen, J., et al. 2007, ApJ, 671, 1329

Nguyen, Q.-Rieu., Jackson, J. M., Henkel, C., et al. 1992, ApJ, 399, 521

Nicholson, R. A., Bland-Hawthorn, J., \& Taylor, K. 1992, ApJ, 387, 503

O’Sullivan, E., Combes, F., Hamer, S., et al. 2015, A\&A, 573, A111

Ostriker, J. P., Choi, E., Ciotti, L., et al. 2010, ApJ, 722, 642

Ott, J., Meier, D. S., McCoy, M., et al. 2013, ApJL, 771, L41

Peng, E. W., Ford, H. C., Freeman, K. C., et al. 2002, AJ, 124, 3144

Phillips, T. G., Ellison, B. N., Keene, J. B., et al. 1987, ApJ, 322, 73

Querejeta, M., Schinnerer, E., García-Burillo, S., et al. 2016, A\&A, 593, A118

Quillen, A. C., Brookes, M. H., Keene, J., et al. 2006, ApJ, 645, 1092

Quillen, A. C., de Zeeuw, P. T., Phinney, E. S., et al. 1992, ApJ, 391, 121

Quillen, A. C., Graham, J. R., \& Frogel, J. A. 1993, ApJ, 412, 550

Quillen, A. C., Neumayer, N., Oosterloo, T., et al. 2010, PASA, 27, 396

Roberts, M. S. 1970, ApJ, 161, 9

Rodriguez-Franco, A., Martin-Pintado, J., \& Fuente, A. 1998, A\&A, 329 1097

Rydbeck, G., Wiklind, T., Cameron, M., et al. 1993, A\&A, 270, 13

Salomé, P., \& Combes, F. 2003, A\&A, 412, 657

Salomé, Q., Salomé, P., Combes, F., et al. 2016, A\&A, 586, A45

Santoro, F., Oonk, J. B. R., Morganti, R., et al. 2015, A\&A, 575, L4

Sarma, A. P., Troland, T. H., \& Rupen, M. P. 2002, ApJ, 564, 696

Schilke, P., Walmsley, C. M., Pineau Des Forets, G., et al. 1992, A\&A, 256, 595

Seaquist, E., \& Bell, M. ApJ, 364, 94

Shirley, Y. L. 2015, PASP, 127, 299S

Sternberg, A., \& Dalgarno, A. 1995, ApJS, 99, 565

Strong, A. W., Bloemen, J. B. G. M., Dame, T. M., et al. 1988, A\&A, 207, 1

Struve, C., Morganti, R., Oosterloo, T. A., et al. 2010, PASA, 27, 390

Tingay, S., Jauncey, D., Reynolds, J., et al. 1998, AJ, 115, 960

van der Hulst, J. M., Golisch, W. F., \& Haschick, A. D. 1983, ApJ, 264, 37

Verdoes Kleijn, G. A., Baum, S. A., de Zeeuw, P. T., et al. 1999, AJ, 118, 2592

Wiklind, T., \& Combes, F. 1997, A\&A, 324, 51

Wilson, A. S., \& Colbert, E. J. M. 1995, ApJ, 438, 62

Wilson, T. L., \& Rood, R. 1994, ARA\&A, 32, 191

Wootten, A., Bozyan, E. P., Garrett, D. B., et al. 1980, ApJ, 239, 844

Young, J. S., \& Sanders, D. B. 1986, ApJ, 302, 680 